\title{
On the nature of self-field critical current in superconductors and its use as a probe of the superfluid density
}

by

Wayne Philip Crump

A thesis

submitted to the Victoria University of Wellington in fulfilment of the requirements for the degree of Doctor of Philosophy.

Victoria University of Wellington 2019 



\begin{abstract}
Superconductors are used in many applications where large electrical currents are needed. This is due to their ability to transport an electric current without resistance. There is however a limit to the magnitude of current that can be conducted before dissipation starts to occur. This is known as the critical current and is a topic of great interest in applied superconductivity.

For type II superconductors, it is well known that vortex motion plays a role in the determination of the in-field critical current. This has led great effort in engineering the microstructure of these superconductors to hinder the motion of vortices and enhance their critical currents. However the self-field critical current (when there is no applied external field) generally does not see any enhancement due to efforts to pin vortex motion.

The work here examines the behaviour of the self-field critical current in thin-film and cylindrical wire superconductors of many different superconductor types and sizes. It is found that a critical state is reached when the current density at the surface of the sample reaches the magnitude of $B_{c} / \mu_{0} \lambda$ for type I and $B_{c 1} / \mu_{0} \lambda$ for type II superconductors regardless of the size and material type. This finding shows that there is a fundamental limit to the self-field current density that cannot be enhanced by engineering the microstructure and is essentially of thermodynamic origin.

The result also sets up the self-field critical current density as a probe of the superfluid density. This was explored in many different superconductor types by considering the temperature dependence of the self-field critical current. The ground-state magnetic penetration depth, groundstate energy gap and specific heat jump at the critical temperature were
\end{abstract}


key thermodynamic parameters extracted from the critical current data. For a very large number of superconductors the extracted parameters in general matched well with literature values measured using conventional but much more complex techniques.

A result inferred from the critical state was that the current distribution across the width of a rectangular superconductor would be uniform, contrary to expectations of the Meissner state. This was tested by measuring the perpendicular magnetic field resulting from a transport current in a superconducting tape as it reached the critical state. It was indeed found that the current distribution is uniform across the width.

The self-field critical current was also measured in $\mathrm{YBa}_{2} \mathrm{Cu}_{3} \mathrm{O}_{y}$ samples with $\mathrm{Zn}$ impurities to measure the superfluid density and further test the self-field critical current as a measure of superfluid density and in particular explore whether it follows the canonical dependence on the transition temperature observed for superconductors with $d$-wave symmetry. Here the critical current was found to reduce as more impurities were added and indeed this matched its expected canonical reduction, following the superfluid density as $J_{c}(s f) \propto \rho^{3 / 2}$.

These results taken together support the unexpected existence of a fundamental limit in the self-field critical current, which is thermodynamic in origin. 


\section{Acknowledgments}

I would like to thank my wife for her encouragement and support.

I would like to thank both of my supervisors, Prof. Jeffery Tallon and Dr. James Storey for their guidance and help during my PhD. I would also like to thank Dr. Evgeny Talantsev for his close collaboration. Without the help of these people I could not have made the progress I did.

I would also like to thank the staff at the Robinson Research Institute for their help in using lab equipment and setting up experiments, in particular Stuart, Nick and Shen.

I also would like to thank Dr Michael Eisterer and his group at the Technical University of Vienna for helping out with additional critical current measurements in their laboratory.

This work was funded by the MacDiarmid Institute and the Marsden fund. 


\section{Preface}

As is the norm in scientific research in this age, collaboration is a key component and the use of "we" in this thesis seeks to honour that fact. The work presented here would not be possible without a close and sustained collaboration with Prof. Jeffery Tallon and Dr. Evgeny Talantsev. This work has led to several publications seen below, a few of which the thesis is based on.

My responsibilities included programming the fitting software which was made difficult by the use of integrals in the various equations. I developed the treatment of anisotropy and the relevant geometrical form factors for the general case of rectangular conductors (Chapter 8). I also fitted the data, participated in all discussions in developing the work and concepts and identified the key result in Chapter 7 that the thermodynamic critical current lies below the engineering critical current and therefore is of fundamental origins.

Other work was also carried out that is not included in the thesis. This includes work investigating the Neel temperature in the Re-123 compounds using muon spin relaxation and Raman two-magnon scattering in order to correlate physical and magnetic properties. This is being done in collaboration with Dr Amit Keren with two further publications to be expected in the near future.

- E. F. Talantsev, W. P. Crump, J. G. Storey, and J. L. Tallon, London penetration depth and thermal fluctuations in the sulphur hydride 203 K superconductor, Ann. Phys. (Berlin), vol. 529, no. 3, p. 1600390 
(2016).

- E. F. Talantsev, W. P. Crump, J. Island, Y. Xing, Y. Sun, J. Wang, and J. L. Tallon, On the origin of critical temperature enhancement in atomically thin superconductors, 2D Materials, vol. 4, no. 2, p. 025072 (2017).

- E. F. Talantsev, W. P. Crump, and J. L. Tallon, Thermodynamic prameters of single or multiband superconductors derived from self-field critical currents, Ann. Phys. (Berlin), vol. 529, no. 12, p. 1700197 (2017).

- E. F. Talantsev, N. M. Strickland, S. C. Wimbush, and W. P. Crump, The onset of dissipation in high-temperature superconductors: Selffield experiments, AIP Advances, vol. 7, p. 125230 (2017).

- E. F. Talantsev, W. P. Crump, and J. L. Tallon, Universal scaling of the self-field critical current in superconductors: from sub-nanometre to millimetre size, Scientific Reports., vol. 7, no. 10010, p. 1 (2017).

- E. F. Talantsev, W. P. Crump, and J. L. Tallon, Two-band induced superconductivity in single-layer graphene and topological insulator bismuth selenide, Superconductor Science and Technology, vol 31, no. 1, p. 015011 (2017).

- E. F. Talantsev, A. E. Pantoja, W. P. Crump, and J. L. Tallon, Current distribution across type II superconducting films: a new vortex-free critical state, Scientific Reports, vol. 8, p. 1716 (2018). 


\section{Contents}

$\begin{array}{lll}1 & \text { Introduction } & 1\end{array}$

2 Background 5

2.0.1 The Bardeen-Cooper-Schrieffer theory of supercon-

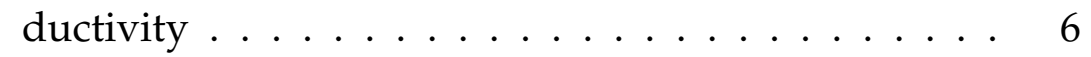

2.0 .2 Meissner effect and magnetic penetration depth . . . 10

2.0 .3 Critical currents in superconductors . . . . . . . . 10

2.0 .4 Ginzburg-Landau theory . . . . . . . . . . . . . 14

2.0 .5 Magnetic field penetration in type I superconductors 16

2.0 .6 Vortices in type II superconductors. . . . . . . . . . . 18

2.0 .7 Critical currents in type II superconductors . . . . . . 19

3 Methods 23

3.1 MOD-TFA YBCO+Zn thin-films. . . . . . . . . . . . . . . 23

3.1 .1 Thin film synthesis . . . . . . . . . . . . . . . . . 24

3.1 .2 Current bridge fabrication . . . . . . . . . . . . 26

3.2 Critical current measurements . . . . . . . . . . . . . . . 27

3.2 .1 Critical current measurement system . . . . . . . . 28

3.2 .2 Measurement process . . . . . . . . . . . . . . . . 29

3.3 Fitting Algorithm and errors in fitting parameters . . . . . . 31

4 Universal scaling behaviour in the self-field critical current 33

4.1 Critical current behaviour across a wide range of sample sizes 33 
4.2 Normalized surface current and surface field . . . . . . . . . 37

5 Temperature dependence of self-field critical current and pene-

tration depth 45

5.1 Tin - high aspect ratio . . . . . . . . . . . . . . 46

5.2 Tin - small aspect ratio . . . . . . . . . . . . . . 46

$5.3 \mathrm{BaKBiO}_{3} \ldots \ldots \ldots \ldots \ldots \ldots \ldots$

6 Deriving thermodynamic parameters from self-field critical current 51

6.1 Model for temperature dependent self-field critical currents 52

6.1.1 Semiclassical BCS expression for the superfluid density ....................... 53

6.1 .2 Energy gap temperature dependence . . . . . . . 54

$6.2 s$-wave superconductors . . . . . . . . . . . . . . . 54

$6.3 d$-wave superconductors . . . . . . . . . . . . . . . . 59

6.4 Nanowire superconductors ... . . . . . . . . . . . 61

7 Perpendicular Field Distribution measurements on superconduct-

\begin{tabular}{|cc|}
\hline ing tapes & 69
\end{tabular}

7.1 Experimental setup . . . . . . . . . . . . . . . 70

7.1 .1 Superconducting tapes used . . . . . . . . . . . . . 71

7.2 Measurement data $\ldots \ldots \ldots$. . . . . . . . . 71

7.3 Field distribution from current distribution . . . . . . . . 73

7.4 Brandt and Indenbom fit to data $\ldots \ldots . . \ldots 77$

8 Effects of impurity scattering on critical current 83

$8.1 \quad$ Impurity scattering in $d$-wave superconductors: Theory . . . 83

8.2 YBCO+Zn critical current data . . . . . . . . . . . . . 85

8.3 Conclusions . . . . . . . . . . . . . . . . . 92

9 Deriving the geometric factors using the London equations 93

9.1 Anisotropic London superconductors . . . . . . . . . . . 94 
CONTENTS ix

9.2 Rectangular superconductor with arbitrary aspect ratios . . 95

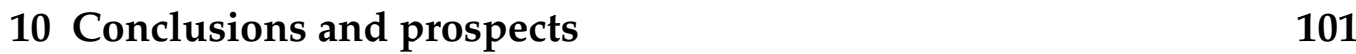

10.1 Summary of results . . . . . . . . . . . . . . . . 101 


\section{List of Figures}

2.1 Discovery timeline of notable superconductors with their transition temperatures. . . . . . . . . . 6

2.2 Superconducting gap temperature dependence for $s$-wave and $d$-wave symmetry. . . . . . . . . . . . . . . 9

2.3 Percentage error between functions $\frac{I_{1}(a / \lambda)}{I_{0}(a / \lambda)}$ and $\tanh (a / 2 \lambda) .13$

2.4 Magnetic field vs critical temperature phase diagram and

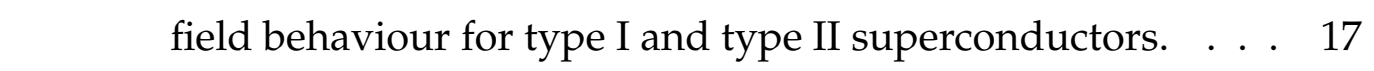

2.5 Effect of pinning on in-field critical current in $\mathrm{YBa}_{2} \mathrm{Cu}_{3} \mathrm{O}_{y}+\mathrm{BaZrO}_{3} .20$

3.1 Photo of $\mathrm{YBa}_{2} \mathrm{Cu}_{3} \mathrm{O}_{y}+\mathrm{Zn}$ sample with a current bridge. . . . 27

3.2 Example of a voltage vs current measurement. . . . . . . . . 30

$4.1 \quad$ Normalized self-field critical current density vs the normalized superconductor dimension. . . . . . . . . . . . 40

4.2 Normalized surface field, surface and global self-field cur-

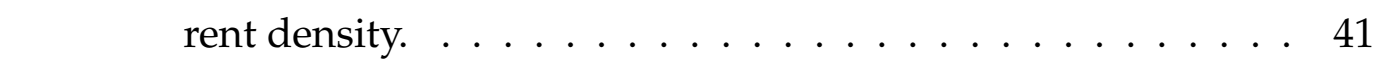

5.1 Comparisons of normalized $\lambda$ for $\mathrm{Sn}$. . . . . . . . . . . . . 47

5.2 Comparisons of normalized $\lambda$ for $\mathrm{Sn} \ldots \ldots$. . . . . . . . 48

5.3 Comparisons of $B_{c 1}$ with $J_{c}(s f, T)$ data for BaKBiO3 . . . . . 49

$6.1 \quad$ Fits of the self-field critical current density for $s$-wave superconductors. . . . . . . . . . . . . 56 
6.2 Comparison of derived parameters from self-field critical current data. . . . . . . . . . . . . . 58

6.3 Fits of the self-field critical current density for $d$-wave superconductors. . . . . . . . . . . . . 60

$6.4 \quad$ Fits of the self-field critical current density for nanowire superconductors. . . . . . . . . . . . . . 63

$7.1 \quad$ Diagram of experiment setup measuring the magnetic field profile. . . . . . . . . . . . . . . 70

7.2 Field profile for various transport currents for a SuperPower Superconducting tape. . . . . . . . . . . . 72

7.3 Calculated field profiles for a Rhoderick and uniform cur-

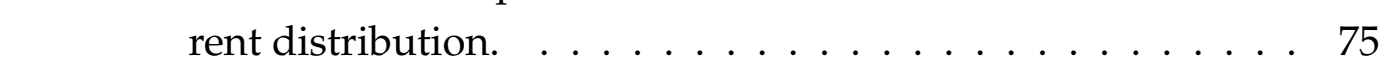

7.4 Comparison of magnetic field profiles both measured and theoretical for several superconducting tapes. . . . . . . . . 78

7.5 Brandt and Indenbom current density distribution . . . . . . 79

7.6 Comparison of the Brandt and Indenbom field profile vs ex- periment. ....................... 80

8.1 Self-field critical current measurements of several YBCO+Zn thin films measured at RRI. . . . . . . . . . . . . . . 86

8.2 Self-field critical current densities of three $\mathrm{YBCO}+\mathrm{Zn}$ thin films samples measured in Vienna. . . . . . . . . . . . . 88

8.3 Canonical superfluid density suppression compared to selffield critical current suppression as a function of impurity concentration. . . . . . . . . . . . . . 91

9.1 Comparison between the approximated geometric factor and that calculated using the London theory. . . . . . . . . . . 98 


\section{List of Tables}

\begin{tabular}{|c|c|}
\hline 4.1 & Calculated $J_{c, n}, J_{s, n}$ and $B_{s, n}$ values for various supercon- \\
\hline & ductors $\ldots \ldots \ldots \ldots \ldots \ldots \ldots$ \\
\hline 4.2 & Calculated $J_{c, n}, J_{s, n}$ and $B_{s, n}$ values for anisotropic YBCO . . 43 \\
\hline 6.1 & Derived parameters from fitting the self-field critical cur- \\
\hline & rent of various $s$-wave superconductors. . \\
\hline 6.2 & Derived parameters from fitting the self-field critical cur- \\
\hline & rent of various $d$-wave superconductors. . . . . . . . . . . . . 66 \\
\hline 6.3 & Derived parameters from fitting the self-field critical cur- \\
\hline & rent of various nanowire superconductors. \\
\hline 7.1 & Manufacturers and dimensions of HTS commercial tapes \\
\hline & used for measuring the field due to the transport current. . . \\
\hline 7.2 & Estimated values of $d_{a}$ and $d_{h}$ for field profile measurements \\
\hline & of superconducting tapes.$\ldots \ldots \ldots 76$ \\
\hline 9.1 & The limits of the geometric factor $\frac{\lambda}{a} \tanh \left(\frac{a}{\lambda}\right)+\frac{\lambda}{b} \tanh \left(\frac{b}{\lambda}\right)$ \\
\hline
\end{tabular}




\section{Chapter 1}

\section{Introduction}

The most distinctive property of a superconductor is the ability to conduct electric current without resistance. This lack of dissipation and therefore absence of heating effect means that superconductors are capable of carrying large current densities on the order of $\mathrm{MA} / \mathrm{cm}^{2}$. This has led to them being used for the generation of magnetic fields for many applications such as in nuclear magnetic resonance (NMR), magnetic resonance imaging (MR) and maglev trains.

However, superconductors have a maximum current they can support before dissipation starts to occur. This is known as the critical current. This property has been a subject of research for many years with an eye to obtaining ever higher critical currents. The critical current is known to be affected by the type of material and its microstructure as well as the temperature and the magnitude and orientation of the applied magnetic field.

In particular the self-field critical current is the critical current of a superconductor when no external field has been applied so that the only field present is that generated by the transport current alone. For type I superconductors it is often taken to be the current at which the self-field reaches the critical field (Silsbee's hypothesis [1]), but more rigorously it is the current at which Cooper pairs depair due to the Doppler shift in their 
energy [2], [3].

For type II superconductors at equilibrium a vortex state exists above the lower critical field $B_{c 1}$. In general currents applied in this state will be dissipative due to the Lorentz force acting on the vortices, causing them to move. Vortex motion can be hindered by "pinning centres", and will only occur when the Lorentz force on the vortex is greater than the pinning force. This means that in general the critical current in type II superconductors must be dependent on the microstructure of the material as the pinning of vortices plays a usually dominant role in its determination. The critical current in type II superconductors is therefore widely viewed as an engineering parameter and is not fundamental. This understanding has led to ongoing increases of critical currents in practical superconductors like $\mathrm{Nb}_{3} \mathrm{Sn}$ and $\mathrm{NbTi}$ over the past 40 years [4, 5], which continue to this day.

This key role for pinning has certainly been found to be true for critical currents when there is an applied field, however when the conductor is under self-field an increase in critical current due to pinning has not been observed. This seems to be not widely recognised and the result is so important that a number of examples are referenced here just for the single, but important case, of $\mathrm{YBa}_{2} \mathrm{Cu}_{3} \mathrm{O}_{7}[6,7,8,9]$. In every case, whether the pinning is introduced as precipitates, or by irradiation, the in-field critical current is significantly enhanced but the self-field critical current is not increased, and in some cases is even slightly reduced.

The question here to be answered is whether vortices play any role in the determination of the self-field critical current in type II superconductors or there exists a fundamental limit not governed by engineering parameters. This work finds that there is indeed a fundamental limit to the self-field critical current, which is a thermodynamic parameter governed only by the superfluid density. This relationship is exploited to establish a new method of determining the ground-state penetration depth and the energy gap magnitude using temperature dependent self-field critical cur- 
rent data.

\section{Chapter synopsis}

A short introduction to many of the concepts needed to understand the work carried out in the thesis is presented in chapter 2. The important concepts of superconductivity are introduced with a focus on self-field critical currents and the factors that affect them.

Chapter 3 outlines how thin film YBCO+Zn samples were made as well as how in house measurements of the critical current density were carried out.

Chapter 4 explores the self-field critical current across samples of many sizes demonstrating universal behaviour where the surface current density always reaches a fundamental limit at the critical state.

In chapter 6 the temperature-dependent relationship established between the self-field critical current and the penetration depth in chapter 4 is used to estimate the ground-state penetration depth, energy gap and specific heat jump at the critical temperature. These parameters are able to be estimated by analyzing temperature dependent self-field critical current data and fitting to an extended Bardeen-Cooper-Schrieffer (BCS) model.

Measurements of the magnetic field distribution around a thin-film superconductor are reported in chapter 7. These measurements were informed by findings in chapter 4 . Here the current distribution across the width of a thin film is shown to become uniform when the critical current is reached.

Chapter 8 presents the measurements of the critical current in thinfilms of $\mathrm{YBCO}+\mathrm{Zn}$. Here an expected reduction in the critical temperature and self-field critical current is found which is quantitatively consistent with a drop expected in the superfluid density. This is an independent test of the central hypothesis that the self-field critical current is a thermodynamic parameter governed only by the superfluid density. 
Overall the results here come together to form a robust case for this hypothesis and this has highly important implications for the electrodynamics of all superconductors - a subject studied for 80 years yet clearly still not fully mature. 


\section{Chapter 2}

\section{Background}

Superconductivity is a low temperature electronic superfluid state found in many materials known as superconductors. Below a critical temperature $T_{c}$ the material is said to be in the superconducting state while above $T_{c}$ the material reverts to the normal state. The change in state is due to the electrons organizing in reciprocal k-space into Cooper pairs and forming a superconducting condensate (known as a superfluid) [10]. The two most notable characteristics that superconductors exhibit are the conduction of dc current without resistance and perfect diamagnetism also known as the Meissner effect [11]. Several good books are avaliable on the subject for example see Tinkham [12] or Poole [13].

The discovery of superconductivity was due to Kamerlingh Onnes in 1911 [14]. He was measuring the electrical properties of mercury at liquid helium temperatures and he observed a sharp drop of the resistance to zero at a temperature of $4.15 \mathrm{~K}$. Since this initial discovery there has been a quest to find materials with ever higher transition temperatures, the progress of which is illustrated in figure 2.1. Currently superconductivity has been observed in many different types of materials including pure metals [14], alloys [15], heavy fermion systems [16], organics [17], cuprates [18], pnictides [19] and hydrides [20]. These materials have a range of critical temperatures from near zero to around $200 \mathrm{~K}$ in the case 
of the $\mathrm{H}_{2} \mathrm{~S}$ system under extreme pressure [20].

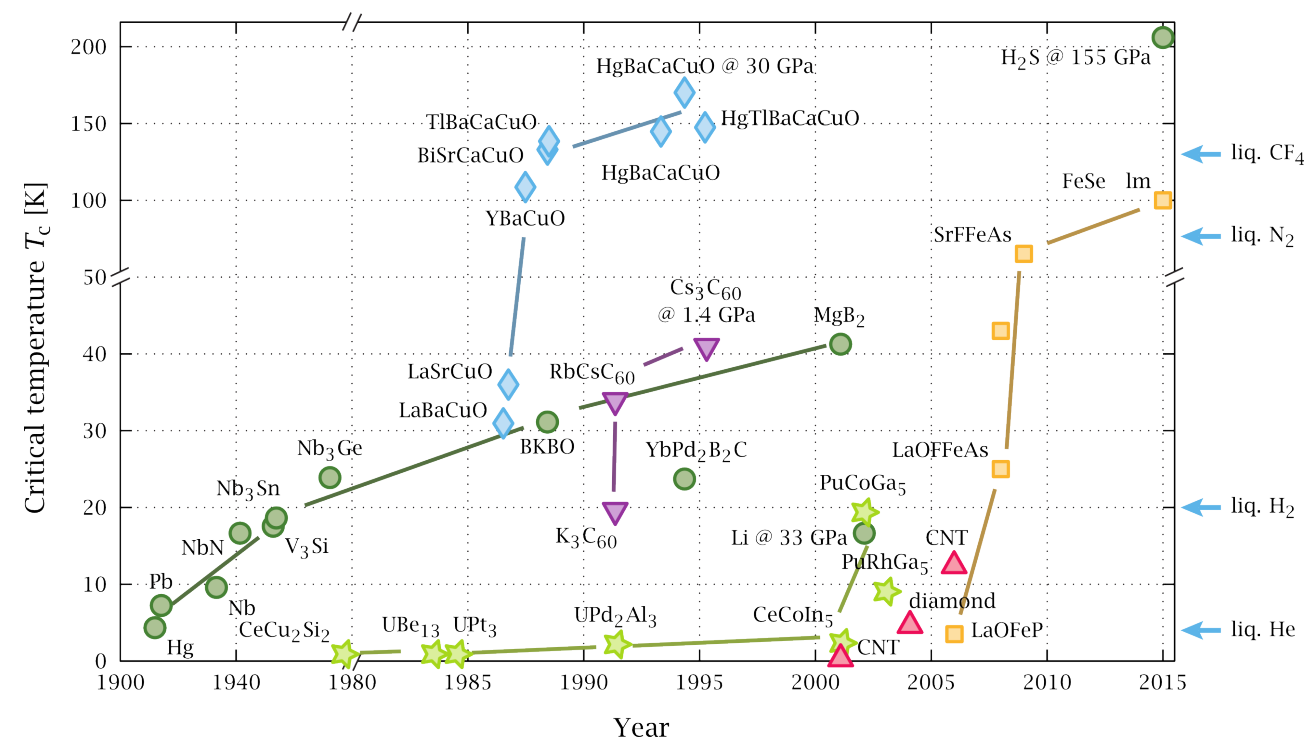

Figure 2.1: Timeline of the discovery and transition temperatures for many known superconductors. Many of the alloys and elemental superconductors are green, the cuprates in blue, the pnctides in orange and the heavy fermion superconductors are lime. The graph is reproduced from Pia Jensen Ray, Figure 2.4 in Master's thesis, "Structural investigation of $\mathrm{La}(2-$ x) $\mathrm{Sr}(\mathrm{x}) \mathrm{CuO}(4+\mathrm{y})$ - Following staging as a function of temperature". Niels Bohr Institute, Faculty of Science, University of Copenhagen. Copenhagen, Denmark, November 2015. DOI:10.6084/m9.figshare.2075680.v2.

\subsubsection{The Bardeen-Cooper-Schrieffer theory of supercon- ductivity}

The microscopic Bardeen-Cooper-Schrieffer (BCS) theory describing superconductivity [10] was not published until 47 years after Onnes' initial discovery. A helpful description of the historical timeline leading up to the BCS theory has been given by Schrieffer [21]. One of the reasons a microscopic theory took so long to complete was that any effort to apply 
perturbation theory to the normal-state electrons would have been unable to reproduce a superconducting state exhibiting the Meissner effect [22]. This is because the Meissner effect arises exclusively from a broken gauge symmetry, completely unrelated to the symmetry of the ground metallic state.

An important aspect of the BCS theory is the Cooper pair which is a pair of electrons bound together due to an attractive interaction between them. In the normal state the electron states are occupied up to the Fermi energy due to the Pauli exclusion principal. If one considers two electrons at the Fermi energy that have an attractive interaction between them, these two electrons will lower their energy by forming a bound state [23]. It is this change of electron behaviour that is needed for superconductivity.

However with enough energy the Cooper pairs can be broken apart. Fluctuations due to temperature will work to break apart Cooper pairs making their formation unstable and so the temperature must be low to form stable Cooper pairs.

In the BCS theory when the superconductor is cooled below the transition temperature, $T_{c}$, it is now energetically favourable for the Cooper pairs to form. The Cooper pairs act like bosons and are able to occupy the same quantum state (or condense into the same state) and this results in the observation of macroscopic quantum behaviour. Also because the electrons around the Fermi energy form the Cooper pairs and are no longer present as single particle quasiparticles, a gap in the density of states forms which is known as the energy gap, $\Delta$.

\section{Pairing mechanism}

As electrons do not naturally attract each other (due to their Coulomb repulsion) there must be some mechanism through which an attraction occurs so that pairing can occur in superconductors. This is known as the pairing mechanism [24]. The BCS theory does not require a specific pairing mechanism, it just requires that there is an attractive interaction between 
the electrons that can overcome the Coulomb repulsion, however the energy scale of the pairing mechanism is important in the BCS theory as it determines several of the superconductor properties. For conventional superconductors the electrons couple with the lattice and can interact via the exchange of a phonon [25] [26].

\section{Energy Gap}

The energy gap, $\Delta(T)$, is the size of the gap formed at $E_{F}$ in the density of states, and it is also the energy range about the Fermi surface where the electrons contributing to the pairing come from. In the BCS theory it can be calculated self-consistently using the equation:

$$
\Delta_{\mathbf{k}}=-\sum_{\mathbf{k}^{\prime}} V_{\mathbf{k}^{\prime}} \frac{\Delta_{\mathbf{k}^{\prime}}}{2 E_{\mathbf{k}^{\prime}}} \tanh \frac{\beta E_{\mathbf{k}^{\prime}}}{2}
$$

where $\beta=\left(k_{B} T\right)^{-1}, E_{\mathbf{k}}=\sqrt{\xi_{\mathbf{k}}^{2}+\Delta_{\mathbf{k}}^{2}}$ where $\xi_{\mathbf{k}}$ are the energies of the electrons in the normal state, and $V_{\mathbf{k k}^{\prime}}$ is the interaction term which is due to the pairing mechanism. It can be seen that the gap can have a $\mathbf{k}$ dependence and its symmetry will be dependent on the interaction term $V_{\mathbf{k k}^{\prime}}$.

A common model considered that applies to many elemental and alloy superconductors is the s-wave weak-coupling model. Here the interaction term is set to be a constant $V_{0}$ up to a cut-off which is the Debye cut-off frequency, $\hbar \omega_{D}$, defined by the Debye temperature $\theta_{D}=\hbar \omega_{D} / k_{B}$. This creates an isotropic gap with $s$-wave symmetry and a ground-state magnitude of $\Delta(0)=2 \hbar \omega_{D} \exp \left(-1 / N(0) V_{0}\right)$ where $N(0)$ is the density of states at the Fermi energy. The gap ratio, $2 \Delta(0) / k_{B} T_{c}$, can be calculated and it is found to be a constant for all weak-coupled superconductors regardless of the phonon energy used for the interaction. The temperature dependece of the gap in the s-wave model is plotted in figure 2.2 as the blue line where it has been normalized to unity.

Another type of pairing symmetry found in cuprate superconductors is $d$-wave symmetry [27]. The energy gap around the Fermi surface is 
anisotropic following the function $\cos (2 \theta)$. The $s$-wave and $d$-wave symmetries have slightly different gap temperature dependences (shown in figure 2.2 however the difference between them are able to be observed more easily in measurements of the penetration depth.

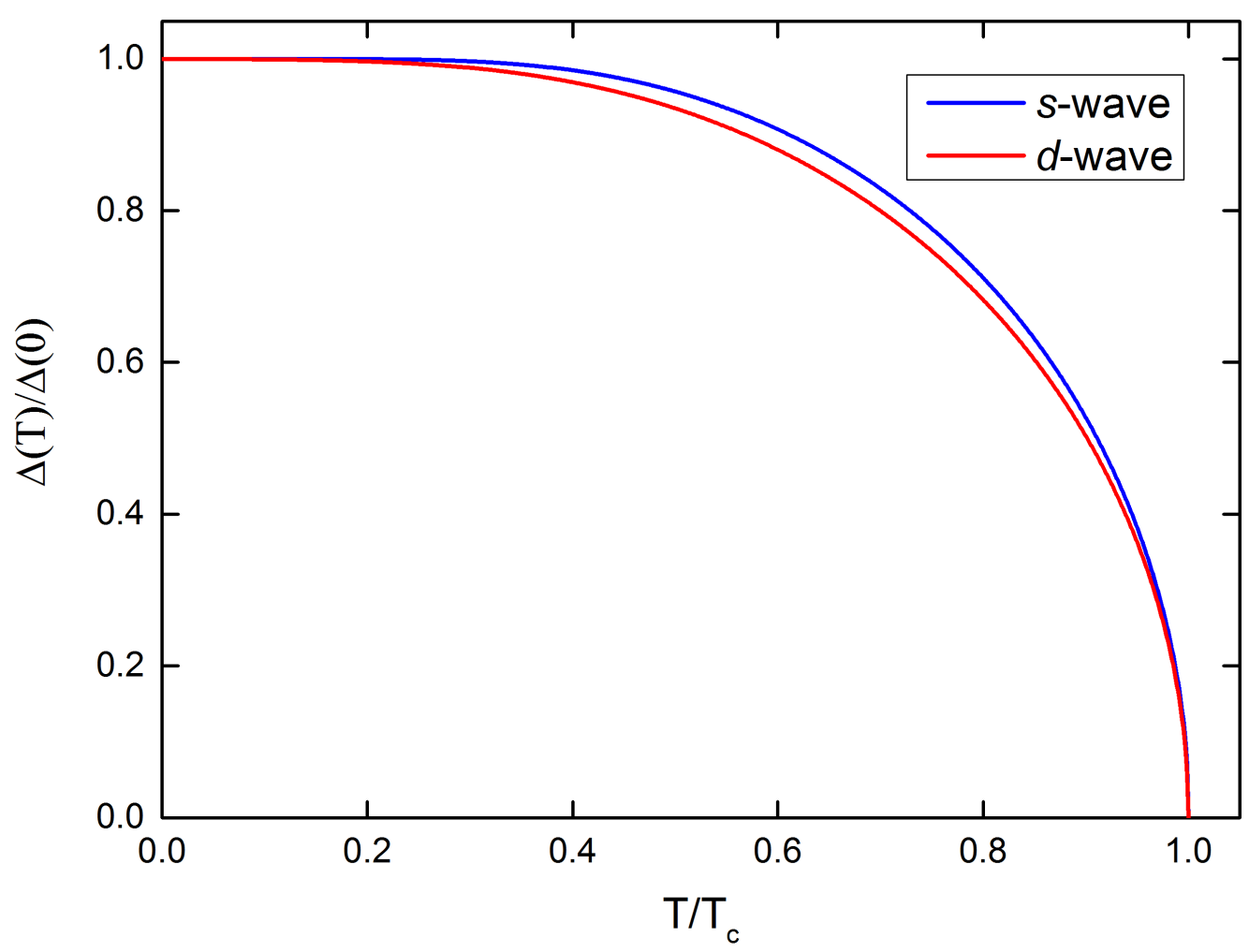

Figure 2.2: The temperature dependence of the superconducting energy gap, $\Delta(T)$, calculated using equation 2.1. For the $s$-wave symmetry (blue line) the interaction term, $V_{\mathbf{k k}^{\prime}}$, is a constant $\left(V_{0}\right)$ up to the cut-off energy $\left(\hbar \omega_{D}\right)$ after which it is zero. For the $d$-wave symmetry (red line) the interaction term is set to be $V_{0} \cos (2 \theta) \cos \left(2 \theta^{\prime}\right)$ [28] [29] up to the cut-off energy $\left(\hbar \omega_{D}\right)$ after which it is zero. 


\subsubsection{Meissner effect and magnetic penetration depth}

As noted, a key behaviour of a superconductor is the expulsion of magnetic field from inside the superconductor when it is in the superconducting state. This is known as the Meissner effect and the superconductor is said to be in the "Meissner state". In the Meissner state the penetration of the magnetic field into the superconductor falls off exponentially [30]. The resulting depth of penetration is small and is given by the magnetic penetration depth or London penetration depth, $\lambda(T)$. This penetration depth is a material dependent property (for example see table 12.1 in [31]).

The expulsion of the field is caused by circulating supercurrents which act to reduce the internal field to zero - a process known as "screening". This will only happen for magnetic fields up a to certain strength where there will be a distinction between two classes of superconductors, type I and type II which are discussed further down. These screening currents also only penetrate to a depth of the order $\lambda$.

A derivation for calculating the penetration depth using the BCS theory is given by Chandrasekhar [32] and an expression for different superconductor types is given in chapter 6 .

\subsubsection{Critical currents in superconductors}

Very soon after the discovery of superconductivity it was found that there were limits to the conduction of current without resistance. It was two years after Onnes' first observation of superconductivity that he discovered the existence of a "threshold" current in $\mathrm{Hg}$ [33]. Increasing the current past this threshold value would result in a resistance reappearing. This became known as the critical current, $I_{c}$, which is the maximum current a superconductor can carry without dissipation.

Usually many factors can determine the $I_{c}$ of a sample such as the particular superconductor itself, the size and shape, the microstructure (e.g. defects or grain boundaries), the temperature $T$, the magnetic field ori- 
entation and strength $\mathbf{B}$ or the existence of competing electronic phases which may serve to weaken the superconductivity [34], [35], [36].

There is an upper limit to the critical current density the is known as the depairing current density. This is the current where the kinetic energy of a Cooper pair exceeds its binding energy $2 \Delta$ causing pair breaking. This depairing current density is given as

$$
J_{d}=\frac{n_{s} e \Delta}{m_{e} v_{f}}=\frac{\phi_{0} \kappa}{2 \sqrt{2} \pi \mu_{0} \lambda^{3}}
$$

where $n_{s}$ is the density of super electrons, $m_{e}$ is the mass of an electron, $v_{f}$ is the Fermi velocity and $p h i_{0}=h / 2 e$ is the flux quantum.

In BCS theory. When these states are perturbed by a transport current the momentum of the electrons changes by the value $d \mathbf{k}$ resulting in the new states $(\uparrow, \mathbf{k}+d \mathbf{k})$ and $(\downarrow,-\mathbf{k}+d \mathbf{k})$. The depairing current is that where the

\section{Distribution of currents and fields inside a superconductor: London the- ory}

The earliest and most simple theory that describes how currents and fields are distributed in superconductors was developed by the London brothers [37]. It is usual to express their two equations as

$$
\frac{\partial \mathbf{J}}{\partial t}=\frac{n_{s} e^{2}}{m} \mathbf{E}, \quad \nabla \times \mathbf{J}=-\frac{n_{s} e^{2}}{m} \mathbf{B}
$$

where $\mathbf{E}$ is the electric field, $\mathbf{B}$ is the magnetic field, $n_{s}$ is the superfluid density, $e$ is the electron charge and $m$ is the electron mass. These equations together with Maxwell's equations determine the fields and currents inside the superconductors and can be considered valid when the superconductor is in the Meissner state.

The result of these equations is that the electric field, magnetic field and current density will all follow a Helmholtz equation:

$$
\nabla^{2} \mathbf{J}=\frac{1}{\lambda^{2}} \mathbf{J}, \quad \nabla^{2} \mathbf{B}=\frac{1}{\lambda^{2}} \mathbf{B}, \quad \nabla^{2} \mathbf{E}=\frac{1}{\lambda^{2}} \mathbf{E}
$$


where $\lambda^{2}=m / \mu_{0} n_{s} e^{2}$ defines the London penetration depth and $\mu_{0}$ is the vacuum permeability. For further analysis later on we will consider two conductor geometries, the first being a wire with a radius $a$ and the second being a thin film of width $2 a$ and thickness $2 b$ with no applied fields.

For a wire of radius $a$ where we are using cylindrical coordinates, $(\rho, \theta)$, the current in the wire will be $\mathbf{J}=J(\rho) \hat{z}$ resulting in the differential equation

$$
\frac{1}{\rho} \frac{\partial}{\partial \rho}\left(\rho \frac{\partial J}{\partial \rho}\right)=\frac{1}{\lambda^{2}} J
$$

which is just a Bessel equation. If we require our solution to be finite everywhere in the superconductor and apply the boundary condition $J(a)=J_{s}$ the solution will be

$$
J(\rho)=J_{s} \frac{I_{0}(\rho / \lambda)}{I_{0}(a / \lambda)}
$$

where $I_{\nu}(z)$ is the modified Bessel function of the first kind. The total current density will be $J_{t}=J_{s} \frac{2 \lambda}{a} \frac{I_{1}(a / \lambda)}{I_{0}(a / \lambda)}$, which we find is well approximated by $J_{t}=J_{s} \frac{2 \lambda}{a} \tanh \left(\frac{a}{2 \lambda}\right)$, a result which does not seem to appear in the literature. The percentage error between the approximation and the true function is plotted in figure 2.3 showing that the only notable error occurs between the values of 1 to 100 for $a / \lambda$. It is interesting to note that the mathematical form of this (surprisingly good) approximation is the same as that for the rectangular case (which is shown further below in equation 2.8) and will prove very useful in our scaling analysis.

For a thin film with thickness $2 b$ and width $2 a$ and $b<<a$ the differential equation is more complicated as it contains second derivatives with respect to the $x$ and $y$ directions, however because we want to consider critical currents we can assume for the time being the current distribution along the width to be constant [38]. This will be justified in chapter 4 . The current in the wire will be $\mathbf{J}=J(y) \hat{z}$ ( $x$ is in the width direction and $y$ is in the thickness direction). This leaves us with the differential equation

$$
\frac{\partial^{2} J}{\partial y^{2}}=\frac{1}{\lambda^{2}} J
$$




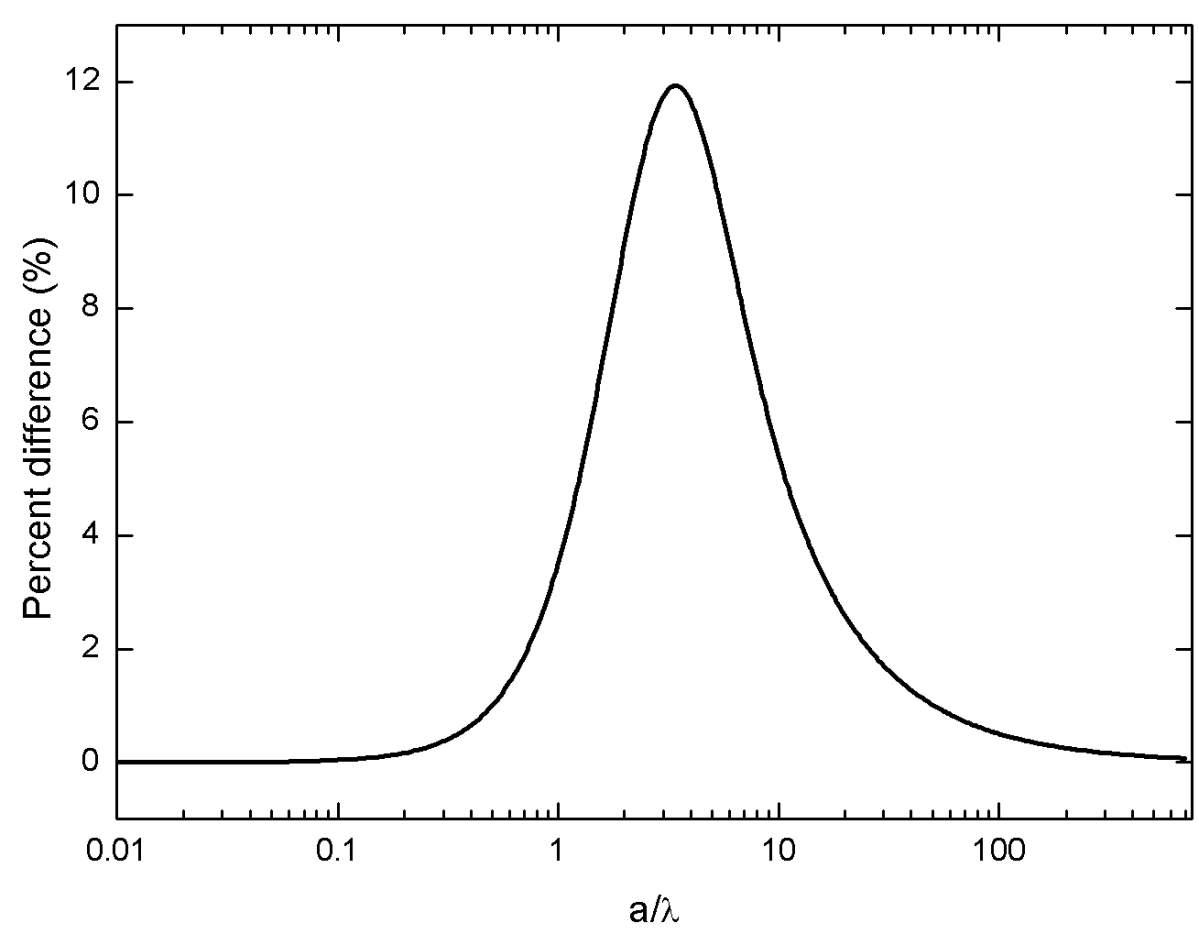

Figure 2.3: The percentage error between the functions $\frac{I_{1}(a / \lambda)}{I_{0}(a / \lambda)}$ and $\tanh (a / 2 \lambda)$ is plotted showing a very good approximation aside from the region 1-100 where the error peaks at $12 \%$

The solution must be an even function $(J(y)=J(-y))$ and have the boundary condition $J(b)=J_{s}$. The solution we obtain is

$$
J(y)=J_{s} \frac{\cosh (y / \lambda)}{\cosh (b / \lambda)}
$$

Here the total current density will be $J_{t}=J_{s}(\lambda / b) \tanh (b / \lambda)$. While this is exact the correspondence with the approximation of the equivalent formula for cylindrical symmetry is notable. 


\subsubsection{Ginzburg-Landau theory}

For the sake of distinguishing the difference between type I and type II superconductors we will first look at the Ginzburg-Landau theory for superconductors [39]. It is a phenomenological theory that describes the superconducting state near the transition point. It can however be derived from the BCS theory [40]. The approach here is taken from chapter 6 in [41].

The free energy functional for a superconductor with order parameter $\psi$ takes the form

$$
\begin{aligned}
f_{s}[\psi]=f_{n} & +\frac{1}{V} \int d^{3} \mathbf{r}\left[\frac{1}{2 m^{*}}\left(-i \hbar \nabla+e^{*} \mathbf{A}\right) \psi^{*} \cdot\left(i \hbar \nabla+e^{*} \mathbf{A}\right) \psi\right. \\
& \left.+\frac{1}{2 \mu_{0}} \mathbf{B}^{2}(\mathbf{r})-\mu_{0} \mathbf{H}(\mathbf{r}) \cdot \mathbf{M}(\mathbf{r})+a \psi \psi^{*}+\frac{b}{2} \psi \psi^{*} \psi \psi^{*}\right]
\end{aligned}
$$

where $f_{s}$ is the free energy density of the superconducting state, $f_{n}$ is the free energy density of the normal state, $a$ and $b$ are parameters that will be material specific where $a$ has a temperature dependence $a=a_{0}\left(T / T_{c}-1\right)$ with $a_{0}$ and $b=b_{0}$ as positive, $V$ is the volume, $\hbar$ is the reduced Planck's constant, $e^{*}=2 e$ is the charge carried by the Cooper pairs and $m^{*}=2 m$ is the mass of the Cooper pairs. The field $\mathbf{H}$ is defined by $\mathbf{H}=\mathbf{B} / \mu_{0}-\mathbf{M}$ where $\mathbf{M}$ is the magnetisation. The order parameter is zero above $T_{c}$ and takes on a complex value below $T_{c}$.

To get the Ginzburg-Landau (GL) equations we require that the free energy be a minimum with respect to variations in the order parameter and vector potential. The GL equations then take the form:

$$
\begin{array}{r}
\frac{1}{2 m^{*}}\left(i \hbar \nabla+e^{*} \mathbf{A}\right)^{2} \psi+a \psi+b|\psi|^{2} \psi=0 \\
\mu_{0} \mathbf{J}=-\frac{i \hbar e^{*}}{2 m^{*}}\left(\psi^{*} \nabla \psi-\psi \nabla \psi^{*}\right)-\frac{e^{* 2}}{m^{*}} \mathbf{A}|\psi|^{2} \\
\mu_{0} \mathbf{J}=\frac{e^{*}}{m^{*}}|\psi|^{2}\left(\hbar \nabla \varphi-e^{*} \mathbf{A}\right)
\end{array}
$$

where in the last equation for $\mathbf{J}$ the complex order parameter takes the form $\psi=|\psi| \exp (i \varphi)$ where both $|\psi|$ and $\varphi$ are real. 
Looking first at the solution to the GL equations deep inside a superconductor, the order parameter takes the value $\left|\psi_{\infty}\right|^{2}=\left(a_{0} / b_{0}\right)\left(1-T / T_{c}\right)=$ $n_{s}^{*}$ where $n_{s}^{*}$ is known as the superfluid density. Two characteristic lengths can also be derived from the GL theory. The first is the coherence length, $\xi=\sqrt{\hbar^{2} / 2 m^{*}|a|}$, the distance over which the order parameter begins to approach its bulk value. The coherence length is related to the average distance between the electrons in a Cooper pair. The second is the London penetration depth and is given as $\lambda=\sqrt{m^{*} / \mu_{0} e^{* 2}\left|\psi_{\infty}\right|^{2}}$.

\section{Type I and II superconductors}

By considering an interface between the normal and superconducting states under a magnetic field a distinction can be made between two groups of superconductors, type I and type II. By considering a plane which can be cut into two half planes, a surface tension $\sigma$ can be defined to be the difference in free energy per unit area between a phase where the whole plane is either normal or superconducting and a mixed phase where half the plane is superconducting and the other half is normal [41]. At the critical field, $H_{c}$, it is found that the surface tension will be either negative or positive depending on the Ginzburg-Landau parameter, $\kappa=\lambda / \xi$ [39]. Its critical value is $1 / \sqrt{2}$ marking the boundary between superconductors that are type I $(\kappa<1 / \sqrt{2})$ and type II $(\kappa>1 / \sqrt{2})$.

When the surface energy is positive, normal-state regions in the superconductor result in an increase in the free energy, however if the surface energy is negative these normal-state regions embedded in the superconductor lower the free energy. For type I superconductors, which have a positive surface energy, when the field reaches the critical field $B_{c}$ the material will return to the normal state. For type II superconductors where the surface energy is negative there will be a lower critical field $B_{c 1}$ above which the superconductor is in the "mixed" or "vortex" state where the core is in the normal state and the circumference of the core forms a surface boundary stabilized by the negative surface energy. There will also 
be an upper critical field $B_{c 2}$ which is the point at which the vortices overlap and the material will return to the normal state (figure 2.4 shows the regions of different phases for both type I and type II superconductors).

Type II superconductors generally have a higher critical temperature and upper critical field compared to the critical temperatures and fields of type I superconductors as well as being more common. The three critical fields can be calculated using the expressions:

$$
B_{c}=\frac{\Phi_{0} \kappa}{2 \sqrt{2} \pi \lambda^{2}}, \quad B_{c 1}=\frac{\Phi_{0}(\ln \kappa+0.5)}{4 \pi \lambda^{2}}, \quad B_{c 2}=\frac{\Phi_{0}}{2 \pi \xi^{2}} .
$$

The thermodynamic critical field is therefore approximately equal to the geometrical mean of $B_{c 1}$ and $B_{c 2}$.

\subsubsection{Magnetic field penetration in type I superconductors}

For type I superconductors in a magnetic field (see for example Tinkham [12] or Ketterson [42]), the geometry will affect how the field penetrates. For example a spherical superconductor in a magnetic field $H_{c}>H_{\mathrm{a}}>$ $2 / 3 H_{c}$ will have penetration at the edges as the field here is increased due to the Meissner effect and the associated distortion of field lines outside the conductor but near its surface. In this way the field at the edges increases above $H_{c}$ causing domains of penetration at the edges. This is the so called intermediate state and is to be distinguished from the mixed state associated with vortices penetrating a type II superconductor.

The strength of applied field needed for a type I superconductor to enter the intermediate state will depend on the geometry of the sample and its orientation in the field.

This intermediate state will also be present in superconductors in a magnetic field which are carrying a current above the depairing value, displaying a so called fractional resistance. The supercurrent remaining inside the core of the conductor is such that it generates the field Hc at its core surface, the rest of the current outside the core is dissipative (causing a 

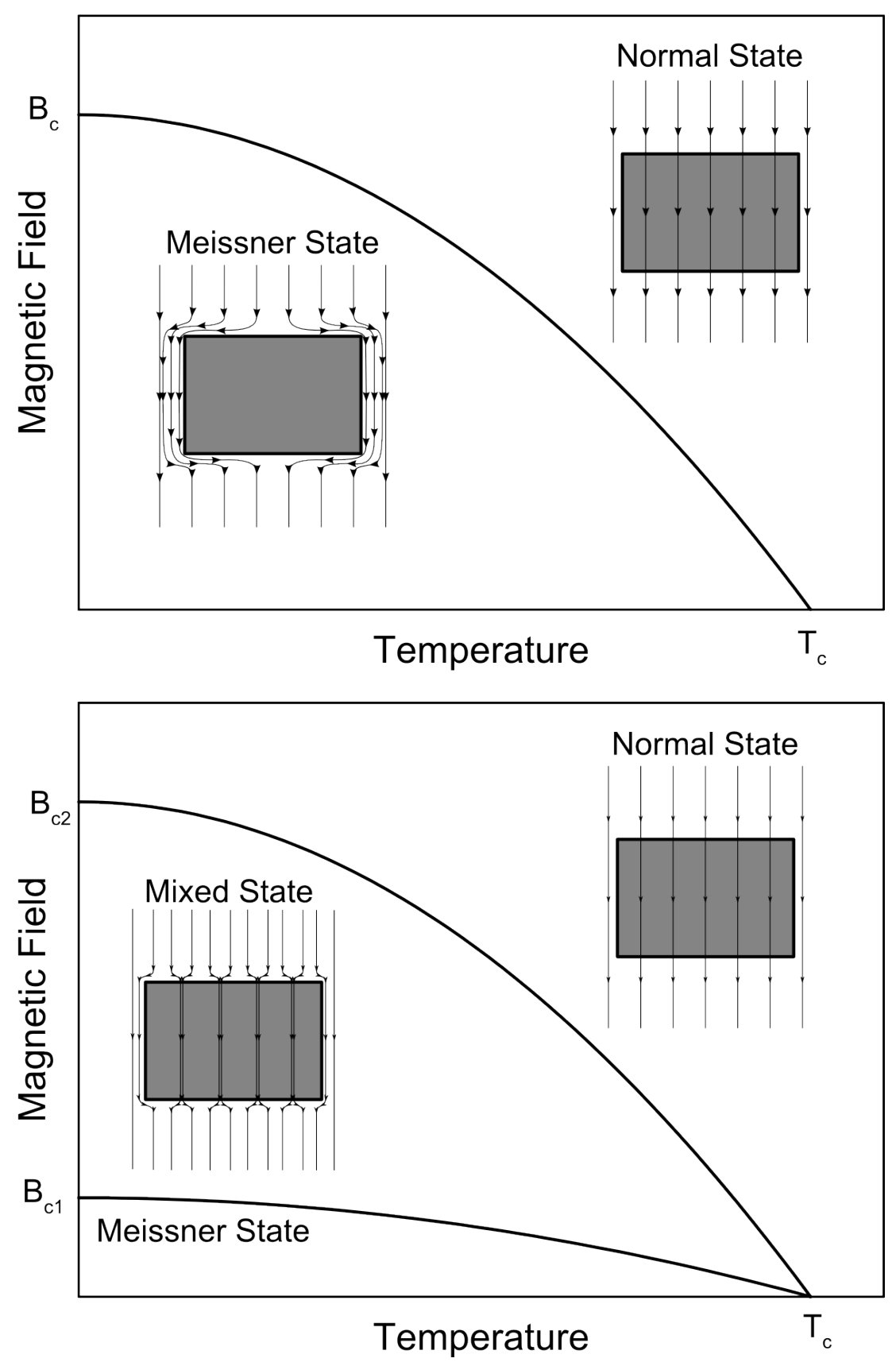

Figure 2.4: The temperature dependence of the critical fields of type II superconductors. Also presented is an illustration of the field distribution for the three different states (Meissner, mixed, and normal). In the mixed state an applied magnetic field will penetrate the sample in thin threads of flux known as vortices. 
resistance to appear) as it is supported only by normal quasiparticles. This situation is particularly relevant to the case of approximately equiaxed rectangular cross-section as discussed later in Chapter refC:GeoFactor.

For cylindrical symmetry the depairing current density is reached simultaneously at all points on the circumference. This means the supercurrent is now confined to a smaller radius and it must therefore exceed the depairing current density and so the conductor catastrophically transitions to the normal state. For a rectangular cross section, the depairing current density is first reached at the edges, while the current density at the middle falls far short of this value and so such a conductor does exhibit an intermediate state with the supercurrent confined to the central portion of the cross-section.

\subsubsection{Vortices in type II superconductors}

Abrikosov predicted in 1957 that in the mixed state the magnetic field penetrates the superconductor as thin threads of magnetic flux [43] illustrated in figure 2.4. These magnetic flux threads take discrete values of flux $\Phi=n \Phi_{0}=n h / 2 e$ where $n$ is an integer, $\Phi_{0}$ is the magnetic flux quantum, $h$ is Planck's constant and $e$ is the charge on an electron. This is due to the fact that the free energy will be minimized when the normal regions are as small in volume as possible whilst having a large surface area. The flux threads will also form a lattice type structure due to the repulsion between different flux threads [43].

\section{Weak-links}

It is well known that materials and thin-films are often not perfectly crystalline, exhibiting discontinuities in their structures such as dislocations, voids and grain boundaries. In a superconductor the critical current may be sensitive to these defects, especially if the current must flow across them. In particular a grain boundary presents a potential barrier that the 
supercurrent must tunnel through, which in general results in a suppression of the current.

The term weak-link generally refers to a junction between two bulk superconducting regions [44]. The junction may be conducting or insulating as long as Cooper pairs can tunnel through it. The presence of weak-links in a sample may be enough to suppress the critical current [45] [46]. A broadening of the width of the transition is also expected as the number of weak-links increase in the sample [45].

\subsubsection{Critical currents in type II superconductors}

Critical currents in type II superconductors become more complicated due to the appearance of the mixed state. In the mixed state a current applied perpendicular to the vortices induces a Lorentz force $f_{L}=\mathbf{J} \times \mathbf{B}$ that will act on each vortex. This will cause a movement of vortices and hence the current will now be dissipative due to the work done on the vortices.

However these vortices can be pinned by inhomogeneities in the crystal lattice of the superconductor such as defects or grain boundaries. If there is a flux lattice present it will be pinned in a collective manner by any pinning centres present. This is because of the long range order of the flux lattice. Interactions between vortices ensure that the flux lattice possesses a rigidity. Thus pinning a few vortices can ensure the collective pinning of the entire vortex array. The pinning force will be integrated over the number of pinning centers, whilst the Lorentz force on the lattice depends on the current and is integrated over the total number of vortices (which in turn is determined by the strength of applied field).

This means that the critical state will be determined by a balance equation including the forces on the vortices due to the current and the pinning forces, hence the critical current will depend on the microstructure of the crystal. It has been demonstrated many times in the literature the effect pinning can have on increasing the critical current under an applied mag- 
netic field [47, 48, 49, [50]. This has led to decades of study on how to manipulate the microstructure to bring about the largest currents [5], [4].

Illustrated in figure 2.5 is the effect of adding pinning centres to a superconductor. The data is taken from [6] and it shows how the in-field critical current is increased up to a certain point with added $\mathrm{BaZrO}_{3}$ nanoparticles. The self-field (zero applied field) critical current however does not see an increase. Other studies have also failed to show an increase in the self-field critical current even when the in-field critical current is enhanced [47, 48, 51, 49, 52, 53, 50].

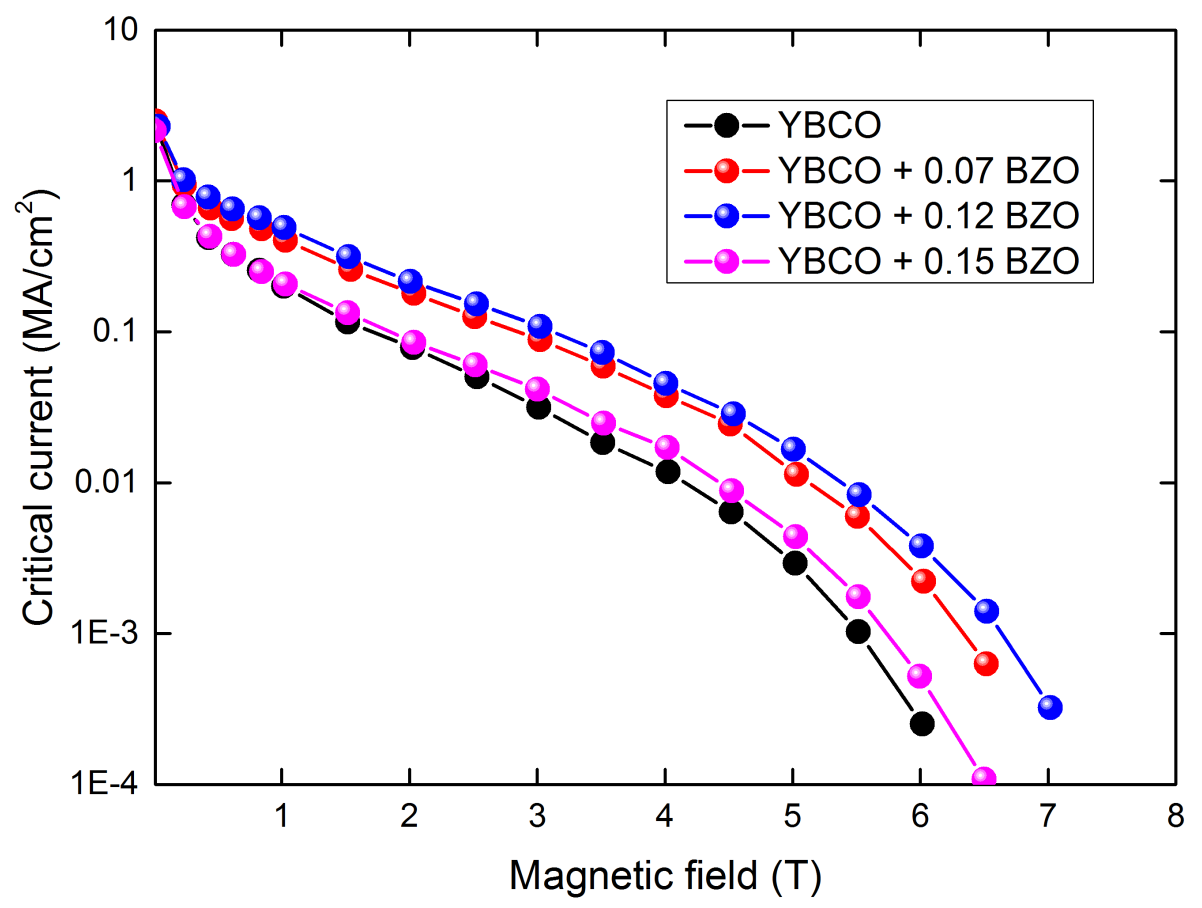

Figure 2.5: In field critical current measurements taken from [6]. The measurements were done on thin-film samples of $\mathrm{YBa}_{2} \mathrm{Cu}_{3} \mathrm{O}_{y}$ with added $\mathrm{BaZrO}_{3}(\mathrm{BZO})$. It can be see that the effect of adding $\mathrm{BaZrO}_{3}$ particles to enhance pinning is only able to increase the critical currents at higher fields, and a noticable difference is not achieved at self-field.

This begs the question, are vorticies important for determining the self- 
field critical current in a superconductor or is there a fundamental limit not governed by engineering parameters that might be thermodynamic in origin? This question is explored in the next chapters where it is answered in the affirmative. The findings also open up the self-field critical current to be used as a probe of the superfluid density, enabling the estimation of several superconductor parameters from a rather straightforward measurement. 


\section{Chapter 3}

\section{Methods}

Much of the focus of this thesis is on the application of a new method to extract fundamental thermodynamic properties of superconductors through the use of critical current data. Much of the data used was taken from the literature by digitizing figures using a free online tool[54], however some of the data was obtained by measurements undertaken in-house including those on superconducting films fabricated in our laboratory. The methods used to prepare these thin film samples and measure their critical currents are described here. [55]

\subsection{MOD-TFA YBCO+Zn thin-films}

Part of the study involved investigating the effects of impurities on selffield critical currents, and thin-film samples of $\mathrm{YBCO}\left(\mathrm{YBa}_{2} \mathrm{Cu}_{3} \mathrm{O}_{y}\right)$ with added $\mathrm{Zn}$ impurities were prepared for this purpose. There were several reasons to use thin-films:

1. Thin films can be made to have the high degree of crystallinity needed for critical current measurements unimpeded by weak links.

2. It is a straight forward process to construct a current bridge on a thin-film and have a well-defined geometry for current transport. 
3. The lab was already set up to make thin-film samples of the type we required.

4. Our critical current measurement equipment was setup optimally for experiments on such thin-film samples.

\subsubsection{Thin film synthesis}

The thin-films were made using the metal-organic deposition (MOD) method using trifluoroacetate (TFA) precursors. The particular method outlined here has also been covered in other publications [56] [57]. Firstly the precursor compounds (Y-TFA, Ba-TFA, Cu-OHP ${ }^{1}$, and $\mathrm{Zn}$-TFA) were weighed out and dissolved one at a time in dry methanol. To ensure each precursor compound was fully dissolved a sonicator was used to mix the solution. Propionic acid was also added to the solution after the first compound to aid the dissolving process.

After all the precursor compounds had been dissolved the next step was to spin coat a substrate with the mixed solution. The trademarked RABiTS (Rolling-Assisted Bi-axially Textured Substrate) was used as it has good lattice matching with YBCO. The base is a Ni-W alloy and on the top are three buffer layers, $\mathrm{Y}_{2} \mathrm{O}_{3}, \mathrm{YSZ}$ and $\mathrm{CeO}_{2}$, each being $75 \mathrm{~nm}$ in thickness. The substrate is pre-cut into a size of $10 \mathrm{~mm} \times 15 \mathrm{~mm}$. The spin coating is done in a glove box with an atmosphere free of oxygen and moisture and after the coating process most of the methanol has evaporated away leaving a gel. This process determines the thickness of the YBCO layer, in our case being around $800 \mathrm{~nm}$. This thickness could be varied by changing the spin speed and concentration of solution.

The samples are then taken out of the glove box in a sealed container and transferred immediately into a furnace for decomposition. The furnace for this part of the process has an atmosphere of flowing oxygen and water vapour. During this process the furnace is heated up to around $450 \mathrm{C}$

\footnotetext{
${ }^{1}$ The chemical formula is a trade secret however a $\mathrm{Cu}$-acetate salt may be used instead.
} 
to remove organic material, leaving behind oxides $\left(\mathrm{Y}_{2} \mathrm{O}_{3}\right.$ and $\left.\mathrm{CuO}\right)$ in both polycrystalline and amorphous phases, as well as Ba-OF.

Next the samples go through a reaction process where the phase of $\mathrm{YBa}_{2} \mathrm{Cu}_{3} \mathrm{O}_{y}$ is formed. This is done under a controlled partial pressure of oxygen and water vapour. The samples go through a proprietary temperature ramping process. During the initial ramp when the temperature rises above $525 \mathrm{C}$ the $\mathrm{Ba}-\mathrm{OF}$ converts to $\mathrm{BaF}_{2}$ and above $720 \mathrm{C}$ the $\mathrm{BaF}_{2}$ reacts with water to make $\mathrm{BaO}$ and $2 \mathrm{HF}$. The $\mathrm{HF}$ is removed leaving behind the $\mathrm{BaO}$ to take part in the formation of $\mathrm{YBCO}$ which is done at $788 \mathrm{C}$ in our case. The temperature ramp rate has to be optimized with respect to the ratio of $\mathrm{BaF}_{2}: \mathrm{BaO}$ at each temperature for the formation of epitaxial thin-films with the c-axis aligned vertically, normal to the substrate.

After the reaction process a silver capping layer was deposited on the top using evaporation. The film is placed inside a vacuum chamber with a portion of silver wire inside a coil of tungsten wire suspended above the sample. The tungsten coil is heated causing the silver wire to evaporate. Some of the evaporated silver vapour condenses on top of the film building a layer of silver. This was done to seal off the YBCO layer from reacting with the atmosphere as well as providing a way of later soldering on electrical contacts for measurements. The silver also provides an alternate current path during measurements for when the current exceeds the critical current. If there was no alternate current path the film would heat up quickly burning the current bridge. In this way the silver layer helps to reduce sample heating. The thickness of this layer was generally around 1-3 $\mu \mathrm{m}$.

To acquire the desired oxygen stoichiometry a further annealing in oxygen atmosphere was carried out. The samples were heated up to $350^{\circ} \mathrm{C}$ and then slowly cooled down to room temperature. This ensured they had an oxygen content close to $y=7$. The annealing was done after the silver deposition. This was to nullify the effect of any sample heating that may have occurred during the silver deposition process. 
As a check of the doping state, or oxygen stoichiometry, measurements of the Seebeck coefficient [58] were made of a few samples. These measurements indicated that using the same annealing conditions resulted in similar doping states. The measured TEP only changed slightly from around $-4 \mu \mathrm{V} / \mathrm{K}$ in the $\mathrm{YBCO}$ film without $\mathrm{Zn}$ impurities to around -3 $\mu \mathrm{V} / \mathrm{K}$ in samples with $1 \%$ and $2 \% \mathrm{Zn}$ content.

\subsubsection{Current bridge fabrication}

For the purpose of critical current measurements the thin-films were patterned with a current bridge. This ensures we are able to calculate the critical current density as the geometry through which the current flows will be well defined. The bridge was constructed by using techniques from photolithography. Photoresist (AZ 600MIF) was deposited on the surface and UV light was exposed through a bridge pattern onto the thin-film. The exposed photo resist was then removed leaving behind the bridge pattern on the film. The exposed silver was then etched chemically using a solution of hydrogen peroxide and ammonia in water, thus exposing the YBCO layer which was also etched chemically using ethylenediaminetetraacetic acid.

The length of the current bridges was $5 \mathrm{~mm}$ and the widths used were either $1.25 \mathrm{~mm}$ or $0.5 \mathrm{~mm}$ depending on the $\mathrm{Zn}$ doping amount with the bridge width determining the absolute magnitude of the critical current. The small bridge widths are needed to reduce the critical current to a level that can be measured using the equipment avaliable. If no bridge was used with the film having a width of $1 \mathrm{~cm}$ it would take a current above $1400 \mathrm{~A}$ to reach the critical current density for YBCO. The critical current density is calculated by dividing the current by the cross sectional area (thickness of the YBCO layer multiplied by the width of the current bridge). A typical bridge is shown in figure 3.1 .

For a measurement of the current bridge YBCO thickness, the silver 


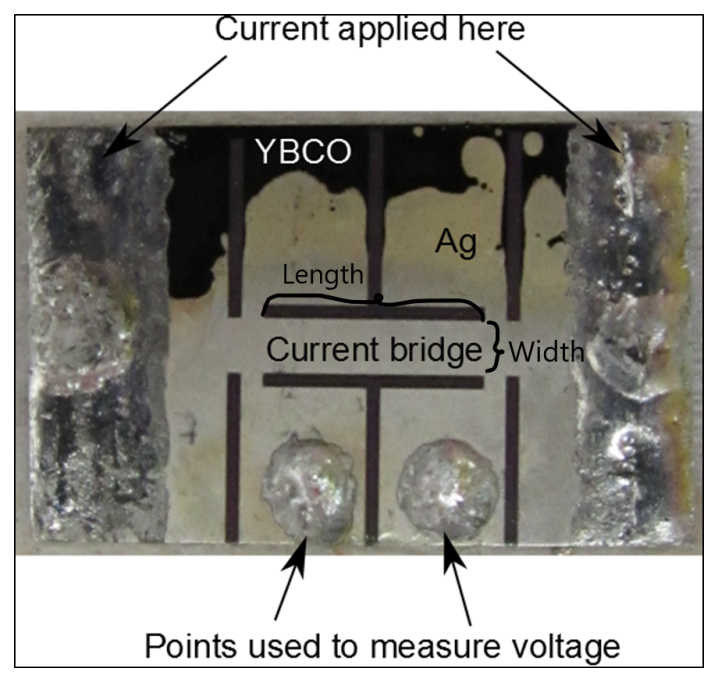

Figure 3.1: A photo of one of the $\mathrm{YBCO}+\mathrm{Zn}$ samples used. The current bridge can be seen as well as contacts where current and voltage leads were soldered on. Some of the silver capping layer has been etched away to reveal the YBCO layer underneath. Both the silver and YBCO has been etched away where the thick lines (bridge cuts) are to define the bridge.

was first etched away uncovering the YBCO layer. The measurement of the current bridge thickness was done using a DekTak stylus profiler instrument. A pin is lowered to the surface being profiled, and changes in the height of the pin are measured as it is moved along the surface. The difference in height between the areas where the YBCO has been etched away (bridge cuts) and where it remains (the middle of the bridge where the current flows) can be used to obtain the YBCO thickness.

\subsection{Critical current measurements}

There are several methods for measuring critical currents in superconductors [59], including magnetisation or transport current approaches, however the 4 point transport method was chosen for this study. This method is the most direct way to measure the response of the superconductor as 
current is pushed through. Magnetisation measurements are complicated by the presence of trapped flux and geometric factors.

\subsubsection{Critical current measurement system}

The particular experimental setup used in our lab is described well by Strickland et al. [60]. The critical current measurement system was built in-house and has the capability to deliver currents up to $875 \mathrm{~A}$ as well as to rotate the sample in an applied magnetic field of up to $8 \mathrm{~T}$.

The system is cooled using a closed circulating gas system. Helium gas is circulated using a pump and it is cooled using a Sumitomo CH-204S cryocooler powered by a HC-4E1 compressor. The temperatures in the system are monitored and controlled using a Lake Shore Model 336 Cryogenic Temperature Controller. Because it is a closed system, care must be taken when changing samples as to stop any air or moisture getting inside. This is done by having a helium over pressure. If any air or moisture enters the system and freezes, the system must be heated to room temperature and flushed using helium gas.

The sample is mounted on a sample rod to be inserted into the system. The sample rod has temperature sensors along it as well as two $22 \mathrm{~W}$ resistive heaters which allows the sample to be heated or cooled. The control of the sample temperature is usually good enough to obtain an uncertainty of less then $\pm 0.001 \mathrm{~K}$. This is important because for a valid measurement care must be taken to ensure the sample temperature remains constant.

A magnetic field up to $8 \mathrm{~T}$ can be applied using a high temperature superconductor (HTS) split-coil magnet and the sample can be rotated in this field using a stepper motor. A calibrated Hall sensor located directly outside the sample space enables measurement of the field. An electrical current up to $875 \mathrm{~A}$ is supplied by an Agilent 6680A dc power supply. The current is measured across the sample using a precision $0.1 \mathrm{~m} \Omega$ power resistor included in the current circuit whilst the voltage across the sample 
is measured using a custom-built nanovolt amplifier with a gain of 10,000 before being read using a National Instruments USB-6211 multifunction data-acquisition module.

\subsubsection{Measurement process}

For the measurement electrical contacts for current injection and voltage measurement are soldered onto the sample at the positions indicated in figure 3.2 and a temperature sensor is attached to the sample bridge. The temperature is first set to a temperature near but below that of the critical temperature. It is good to run a test measurement to ensure the system is working. The measurement is done by first stabilizing the temperature. Then the current is ramped up in exponential steps measuring the voltage at each step. When the voltage rises over a set value the measurement is automatically stopped, giving a current voltage (I-V) curve. Care must be taken to set an appropriate value for the maximum voltage as the bridge can be destroyed if the voltage is too high. Setting a maximum current is a further option to add another layer of safety if there is for example some short-circuit happening.

For a full measurement an automated process is used that measures I-V curves for given temperature steps down to the lowest temperature (20K) and then back up again. In practice we found there to be little difference between measurements cooling down and heating up.

Since the sample is a superconductor we would expect the voltage to not change until the critical current is reached during a measurement. When this happens the voltage will start to increase rapidly due to dissipation. A typical measurement is seen in figure 3.2 .

In the case of a perfect superconductor a step would be seen in the voltage-current curve with the resistance (the slope) after the step being that of the normal state. However the step is smoothed out because of fluctuations. Also the microstructural properties of the superconductor 
can also cause the transition to broaden. The resulting I-V curve is best fit by a power law which is given by

$$
V=V_{c}\left(\frac{I}{I_{c}}\right)^{n} .
$$

Here $I_{c}$ is the critical current and $n$ is the exponent indicating the quality of the sample [61] where lower values can indicate samples with more granular superconductivity. Generally the value of $n$ is high for low temperature measurements compared to measurements near the critical temperature. This is due to fluctuations becoming important at temperatures near the transition temperature.

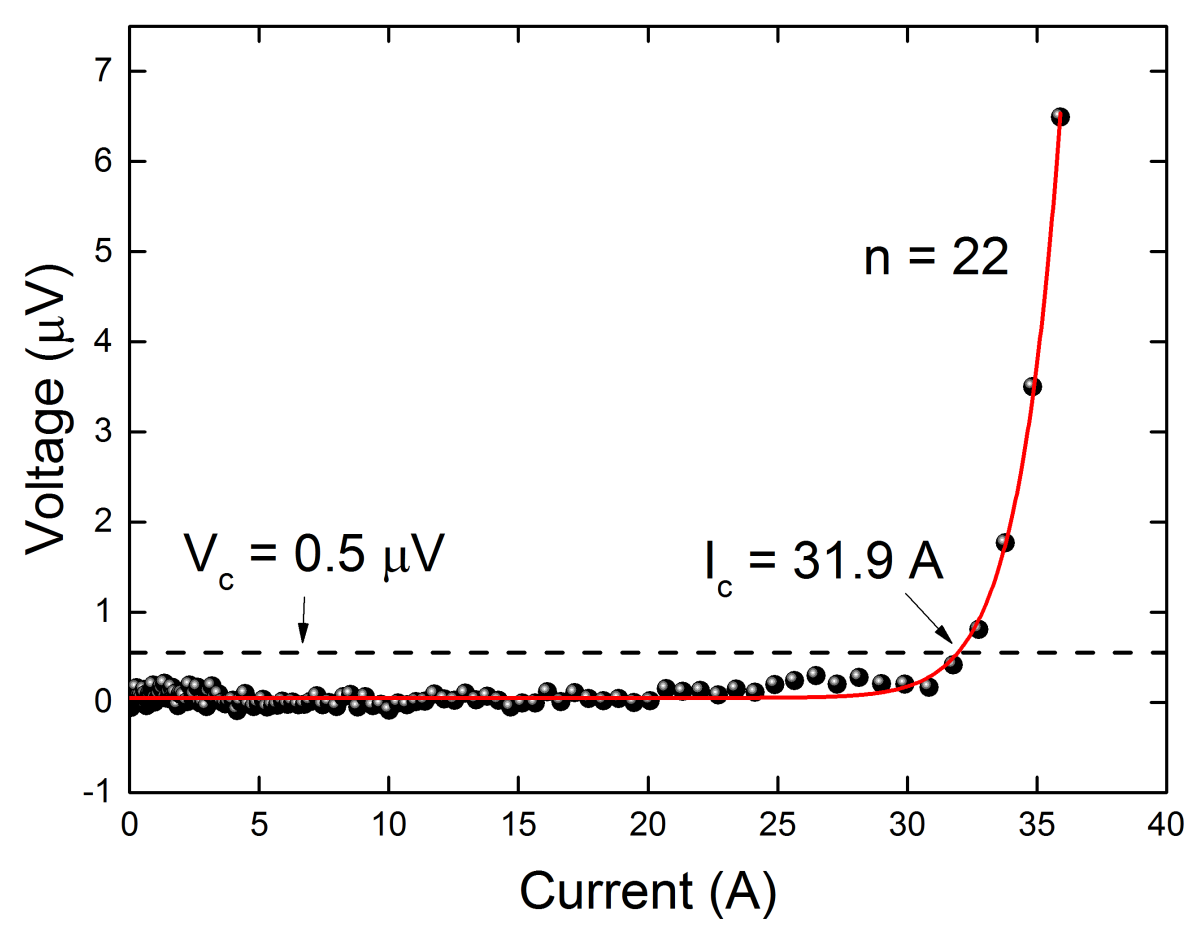

Figure 3.2: An example of a typical voltage vs current measurement. The red line is a power law (equation 3.1 fit to the data with the calculated critical current set by the voltage criterion shown. The value $n$ is the resulting power from the fit.

The value $V_{c}$ is derived from an abitrary electric field criterion $E_{c}$ which 
is usually set to $100 \mu \mathrm{Vm}^{-1}$ as a standard value now used widely in such measurements. As shown in figure 3.2 the critical current will be the current when the voltage reaches $V_{c}=E_{c} l$ where $l$ is the length of the sample bridge. Obviously the smaller $E_{c}$ is the better the estimate of the critical current will be, with the method always overestimating the critical current. One must also consider fluctuations which tend to reduce the value of $n$ close to the transition temperature, causing a larger critical current to be estimated in this region compared with temperatures further away from the transition temperature.

This method was used because it is straight forward and easy to automate, however as we show in chapter 7 this is ultimately an arbitrary criterion-driven method for establishing the critical current. A more fundamental measure is achieved using a surface field criterion.

We then obtain the critical current density, $J_{c}(T)$, by dividing $I_{c}$ by the cross sectional area of the bridge through which the current flows (see figure 3.1].

\subsection{Fitting Algorithm and errors in fitting param- eters}

The fitting of self-field critical current, $J_{c}(s f, T)$, data was carried out using a program set up in Matlab. The program used the least squares method to determine a fit of the data. Because of the nonlinear nature of the fitting functions the matlab function Isqcurvefit was used, where the method of fitting is described next.

In the least squares method the function for fitting the data has several parameters to be determined $\left(\hat{p}=p_{1}, \ldots, p_{n_{p}}\right)$ where in our case these were the critical temperature $T_{c}$, the ground-state gap magnitude $\Delta(0)$, the ground-state penetration depth $\lambda(0)$, and the jump in specific heat at the critical temperature $\Delta C / C$ (see chapter 6). The fitted parameters are found 
by minimizing the sum of squared residuals

$$
S S E(\hat{p})=\sum\left(y_{i}-f\left(\hat{p}, x_{i}\right)\right)^{2}
$$

with respect to the parameters. Here $y_{i}$ are the measured dependent data points $J_{c}\left(s f, T_{i}\right)$, and $x_{i}$ are the measured independent data points $T_{i}$. This minimization was performed using a "subspace trust-region method and is based on the interior-reflective Newton method" described in [62] [63].

The method of minimizing the sum of squared residuals involves calculating the Jacobian

$$
J_{i j}=\frac{\partial f\left(\hat{p}, x_{i}\right)}{\partial p_{j}}
$$

which can be used to calculate the asymptotic covariance matrix $\mathrm{V}=$ $\left(\sigma \mathbf{J}^{T} \mathbf{J}\right)^{-1}$ where $\sigma=S S E /\left(N-n_{p}\right)$. The diagonal elements of $\mathbf{V}$ are the estimated variance of each parameter $\delta_{p_{i}}^{2}=V_{i i}$ and the off-diagonal components are the estimated covariances between parameters $\delta_{p_{i}, p_{j}}^{2}=V_{i j}$. The estimated variance (error) gives an indication of how reliable the fitted parameters are. A large variance with respect to the fitted value would indicate that there may not be enough data points for a good determination of the parameter.

Another way to determine the reliability of the fitted parameters is to find their mutual dependencies. These values can be thought of like this: if the fitted parameters have a high mutual dependency $>0.99$ then this means the parameters can take on a wide range of value pairs (or tuples) and still plot the same fitting function. This will often mean the fitting function has redundant parameters. The mutual dependencies can be calculated using $m d_{p_{i}}=1-1 / V_{i i}\left(V^{-1}\right)_{i i}$. 


\section{Chapter 4}

\section{Universal scaling behaviour in the self-field critical current}

The purpose of this chapter is to demonstrate the totally unexpected universal behaviour of the self-field critical current $J_{c}(\mathrm{sf})$ in many different superconductors over a wide range of length scales. We will see that when the critical current is reached the surface current is always $J_{s}=B_{c} / \mu_{0} \lambda$ for type I superconductors or $J_{s}=B_{c 1} / \mu_{0} \lambda$ for type II superconductors. As a consequence $J_{c}(\mathrm{sf})$ is a thermodynamic quantity, not an engineering quantity. These ideas have been published in the paper [64].

\subsection{Critical current behaviour across a wide range of sample sizes}

Recently it was shown that the self-field critical current density, $J_{c}(\mathrm{sf}, T)$, for thin films where the half thickness, $b$, was less than the penetration depth, $\lambda$, took the following values for type I and type II superconductors 
respectively [65]:

$$
\begin{aligned}
& J_{c}(s f, T)=\frac{B_{c}(T)}{\mu_{0} \lambda(T)} \\
& J_{c}(s f, T)=\frac{B_{c 1}(T)}{\mu_{0} \lambda(T)} .
\end{aligned}
$$

The result for type II superconductors was in particular surprising and was indicative of some sort of universal behaviour. The 2015 paper however focused almost exclusively on data where the thickness of the films was comparable to the penetration depth. A deeper investigation was therefore warranted involving data sets with the superconducting film half-thickness both greater and less than $\lambda$, with the purpose to elucidate more clearly the situation. In the event nearly eight orders of magnitude of sample size was covered.

To explore this relationship more fully we wish to scale or normalize both the self-field critical current density and the conductor half thickness, $b$, by their natural units so as to compare the behaviour of different superconductor types and materials over different length scales.

To begin with the half-thickness is normalized using the penetration depth. As for the current density, $J$, for type I superconductors the natural unit for current density is $H_{c} / \lambda$, where we recall that $H_{c}$ is the thermodynamic critical field. A consideration of equation 4.1 suggests we should use $H_{c 1} / \lambda$ to normalize the current density of type II superconductors.

The normalized critical current density can thus be calculated using the equations

$$
\begin{aligned}
J_{c, n}(T) & =\frac{J_{c}(s f, T)}{B_{c}(T) /\left(\mu_{0} \lambda(T)\right)} \\
J_{c, n}(T) & =\frac{J_{c}(s f, T)}{B_{c 1}(T) /\left(\mu_{0} \lambda(T)\right)}
\end{aligned}
$$

for type I and II respectively. The normalized critical current density provides a comparison between the measured self-field critical current density $J_{c}(s f, T)$ and the self-field critical current density predicted according to equation 4.1 and any size dependent behaviour will be readily exposed. 
To calculate $J_{c, n}$ we use the ground-state values for both $J_{c}(s f, T)$ and $\lambda(T)$, i.e. the values at $T=0$. The values of $J_{c}(s f, 0)$ can be extrapolated from temperature dependent critical current measurements and our method for extrapolating values of $J_{c}(s f, 0)$ is described in chapter 6. Values of $\lambda(0)$ are taken from the literature with values and references given extensively in [64].

Calculated values of $J_{c, n}$ are shown in table 4.1 and 4.2 (reproduced from table 1 in [64]) along with the references for each dataset used. Figure 4.1 (reproduced from figure 3 in [64]) is a plot of $J_{c, n}$ vs the normalized conductor dimension $(b / \lambda$ for thin films and $a / 2 \lambda$ for round wires where as before $a$ is the wire radius). The line plotted is the function $\frac{\lambda}{b} \tanh \left(\frac{b}{\lambda}\right)$ which we derived from the London equations in chapter 2 and it can be seen that the data points sit nicely and remarkably on this line.

The orange data points are for the YBCO cuprate superconductor where anisotropy is present. For the data points here the current is flowing along the $a, b$ plane with the $c$-axis in the $y$ direction normal to the film. For these points the normalization is by $B_{c 1}(c) / \mu_{0} \lambda_{a b}$ where $B_{c 1}(c)=\phi_{0}\left(\log \kappa_{c}+\right.$ $0.5) / 4 \pi \lambda_{a b}^{2}$ [66]. The geometric normalization of the half thickness is surprisingly $b / \lambda_{c}$, with the line plotted being $\frac{\lambda_{c}}{b} \tanh \left(\frac{b}{\lambda_{c}}\right)$. Justification for this normalization is presented in detail in chapter 9

For the region where $b / \lambda>1$ it can be seen that $J_{c, n}$ reduces as $\lambda / b$ is increased. We see that an increase in superconductor thickness does not result in an equivalent increase in current so therefore the current distribution across the thickness cannot be uniform. The current is confined to the surface of the superconductor with dead-space in between just as predicted by the London theory in the Meissner state. This observation is in contrast to flux-entry models where the current in the critical state is distributed across the entire cross-section due to the ingress of vortices in the mixed state[38].

Also it can be seen here that type II superconductors follow the same behaviour as type I superconductors reinforcing the idea that vortices may 
not play a role in the onset of self-field dissipation. It also indicates that London-Meissner currents are present in type II superconductors at critical current as they also are in type I. This is a new result that was not previously found in [65].

For the region where $b / \lambda<1$ it can be seen that $J_{c, n}$ remains constant at a value of unity essentially reconfirming the main finding of [65].

The datapoints plotted here have a large range of aspect ratios $(a / b$ from 1 to $3 \times 10^{6}$ ) implying that any increase in width results in an equivalent increase in current. This therefore implies that the current distribution across the width at $J_{c}(\mathrm{sf})$ must therefore be uniform. This prediction will be confirmed in chapter 7

The effective aspect ratio is given by $a / \lambda_{P}$ where the Pearl length [67], $\lambda_{P}$, is given by $\lambda_{P}=\lambda^{2} / b$. From the samples considered this spans 10 orders of magnitude from $3.5 \times 10^{-3}$ to $1.3 \times 10^{7}$. For samples with an effective aspect ratio less than 1 a uniform current distribution across the width is expected but for those samples with a ratio above 1 the current is expected to flow mostly in the edges. This particular case $\left(a b>>\lambda^{2}\right.$, $a>>b$ ) is given by Rhoderick and Wilson [68] for the Meissner state:

$$
J(x)=\frac{I}{2 \pi b \sqrt{a^{2}-x^{2}}} .
$$

The result in figure 4.1 shows that $J_{c, n}$ follows a line the depends only on $b$ and not on $a$. If the Rhoderick and Wilson current were correct, we would see $J_{c, n}$ drop off as $1 / a b$ instead of just $1 / b$. It also must be uniform because there is no dependence on $a$. This indicates that even for these samples with an effective aspect ratio above 1 the current distribution across the width is still uniform in contrast to what is predicted in [68].

It can in fact be seen that $J_{c, n}$ does not depend on the width $a$ at all but only on $b / \lambda$. This would indicate that for the whole range of normalized conductor dimensions the current distribution across the width is uniform at the onset of dissipation in self-field. This can be compared with the 
case of round wires where the current distribution in the azimuth direction is necessarily constant by symmetry. The fact that both rectangular and cylindrical conductors lie on the same universal curve shows that in both cases the current distribution is uniform. Measurements of current and field distribution are discussed in chapter 7 .

\subsection{Normalized surface current and surface field}

In the preceding section we saw that the behaviour of the transport current followed that predicted by the London equations. In chapter 2 the current distribution for London-Meissner currents was given for both thin films and round wires. In the case of a constant current across the width the current distribution for a thin film was

$$
J(y)=J_{s} \frac{\cosh (y / \lambda)}{\cosh (b / \lambda)}
$$

where $J_{s}$ is the surface current density. The global current density, $J_{t}$, and surface field, $B_{s}$, can be calculated as

$$
\begin{aligned}
J_{t} & =J_{s} \frac{\lambda}{b} \tanh \left(\frac{b}{\lambda}\right) \\
B_{s} & =\mu_{0} b J_{t}
\end{aligned}
$$

where the latter equation follows from Ampere's law. For a round wire the current distribution, global current density and surface field are calculated as

$$
\begin{aligned}
J(\rho) & =J_{s} \frac{I_{0}(\rho / \lambda)}{I_{0}(a / \lambda)} \\
J_{t} & =J_{s} \frac{2 \lambda}{b} \frac{I_{1}(a / \lambda)}{I_{0}(a / \lambda)} \\
B_{s} & =\frac{\mu_{0} a J_{t}}{2} .
\end{aligned}
$$

where $I_{n}(z)$ is the $n$th order modified Bessel function of the first kind and $b$ in this case is the radius of the wire. As noted, the ratio of Bessel functions $\frac{I_{1}(a / \lambda)}{I_{0}(a / \lambda)}$ can be approximated well using the function $(2 \lambda / a) \tanh (a / 2 \lambda)$. 
Using these equations we can now define two other normalized quantities: the normalized surface current density, $J_{s, n}$, and the normalized surface field, $B_{s, n}$. At critical current the surface current density from equation 4.5 will be $J_{s}=J_{c}(b / \lambda) / \tanh (b / \lambda)$. These can be normalized in the same way as before giving us

$$
\begin{aligned}
J_{s, n} & =\frac{J_{c}}{B_{c 1} / \lambda} \frac{(b / \lambda)}{\tanh (b / \lambda)} \\
B_{s, n} & =\frac{\mu_{0} b J_{c}}{B_{c 1}}
\end{aligned}
$$

where the surface field has been normalized using the critical field $B_{c 1}$. These equations are for type II thin films but it is trivial to derive the equivalent equations for type I superconductors as well as for round wires. These quantities can now be calculated and are plotted in figure 4.2 (reproduced from figure 5 in [64]) where again we have used independently measured values of $\lambda(0)$ for the normalisation. The calculated values of $J_{s, n}$ and $B_{s, n}$ are also shown in table 4.1 and 4.2 along with the references for each dataset used.

The values of $J_{s, n}$ sit on the line of unity indicating that the surface current density at the onset of self-field dissipation is always $B_{c} / \mu_{0} \lambda$ for type I or $B_{c 1} / \mu_{0} \lambda$ for type II superconductors. This result is irrespective of the film width and thickness and is universal in nature, indicating a thermodynamic limit.

The calculated values of $B_{s, n}$ follow the mirror image of the values of $J_{c, n}$. For $b>\lambda$ the surface field is just the critical field, as expected, however for $b<\lambda$ the surface field reduces below the critical field as $b$ decreases. Because the surface field as at or less than $B_{c 1}$ the superconductor must still be in the Meissner state. As stated before this fact which was also found by observing the $1 / b$ drop off in $J_{c, n}$ indicating that the current only flowed at the edges for samples with $b>\lambda$.

This result gives further support to the idea that vortices may not play a role in determining the self-field critical current in rectangular thin films 
and wires because the required field strength is not present. It also indicates that current flowing in a superconductor at self-field may not be limited due to the field but is limited, rather, due to a fundamental threshold current density. This is because the universal criterion found here is that the surface current density takes the same value, but the surface field is varying.

The appearance of a fundamental threshold current density is perplexing. There does exist a kind of fundamental threshold current density known as the depairing current density which is well known to be $B_{c} / \mu_{0} \lambda$. This makes sense for type I superconductors, however for type II superconductors we see that the threshold current density is $B_{c 1} / \mu_{0} \lambda$. Surely there must be some function that describes this threshold current density in both regions, but at this stage it is not known what mechanism would describe this.

In summary a scaling analysis of many superconductors of different effective aspect ratios has shown that the normalized critical current density follows a universal relationship which is given by $J_{c, n}=\frac{\lambda}{b} \tanh \left(\frac{b}{\lambda}\right)$. The $1 / b$ drop off for $b>\lambda$ indicates that the samples are still in the Meissner state. This is further supported by the normalized surface field data showing that the surface field never exeeds $B_{c}$ for type I or $B_{c 1}$ for type II superconductors. This precludes the role of vortices in determining the self-field critical current in type II superconductors, contrary to the established view.

The fact that there was no $a$ dependence indicated that the current distribution across the width in the critical state is uniform, a hypothesis that will be tested later in chapter 7. At the critical state the current density across the surface reaches a critical value given by the equation 4.1 which is universal and is a thermodynamic limit. 


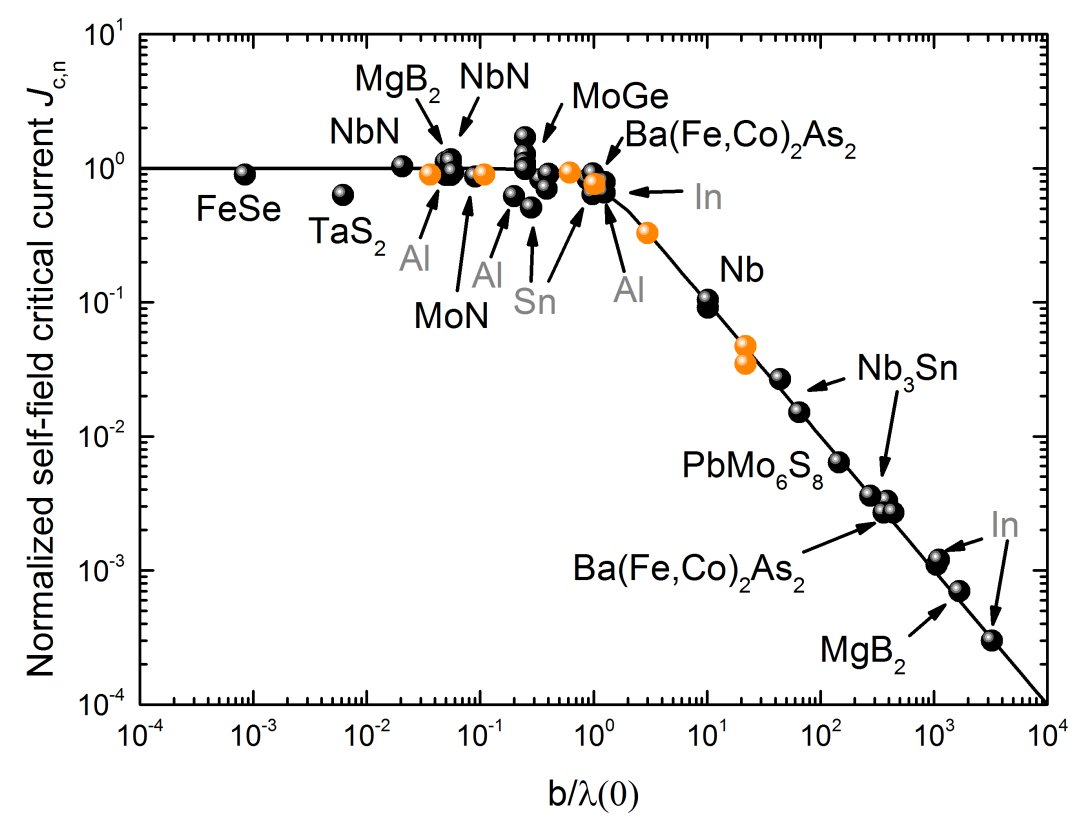

Figure 4.1: Normalized self-field critical current density vs the normalized superconductor dimension ( $b / \lambda$ for thin films and $a / 2 \lambda$ for round wires). The line is the function $\frac{\lambda}{b} \tanh \left(\frac{b}{\lambda}\right)$. The orange data points are the normalized critical current densities for YBCO samples which are plotted vs $b / \lambda_{c}$. The grey labels indicate type I superconductors, whilst the black labels indicate type II superconductors. This graph is produced from tables 4.1 and 4.2 and can be found as supplementary figure 4 in [64] without the $J_{s, n}$ and $B_{s, n}$ data. 


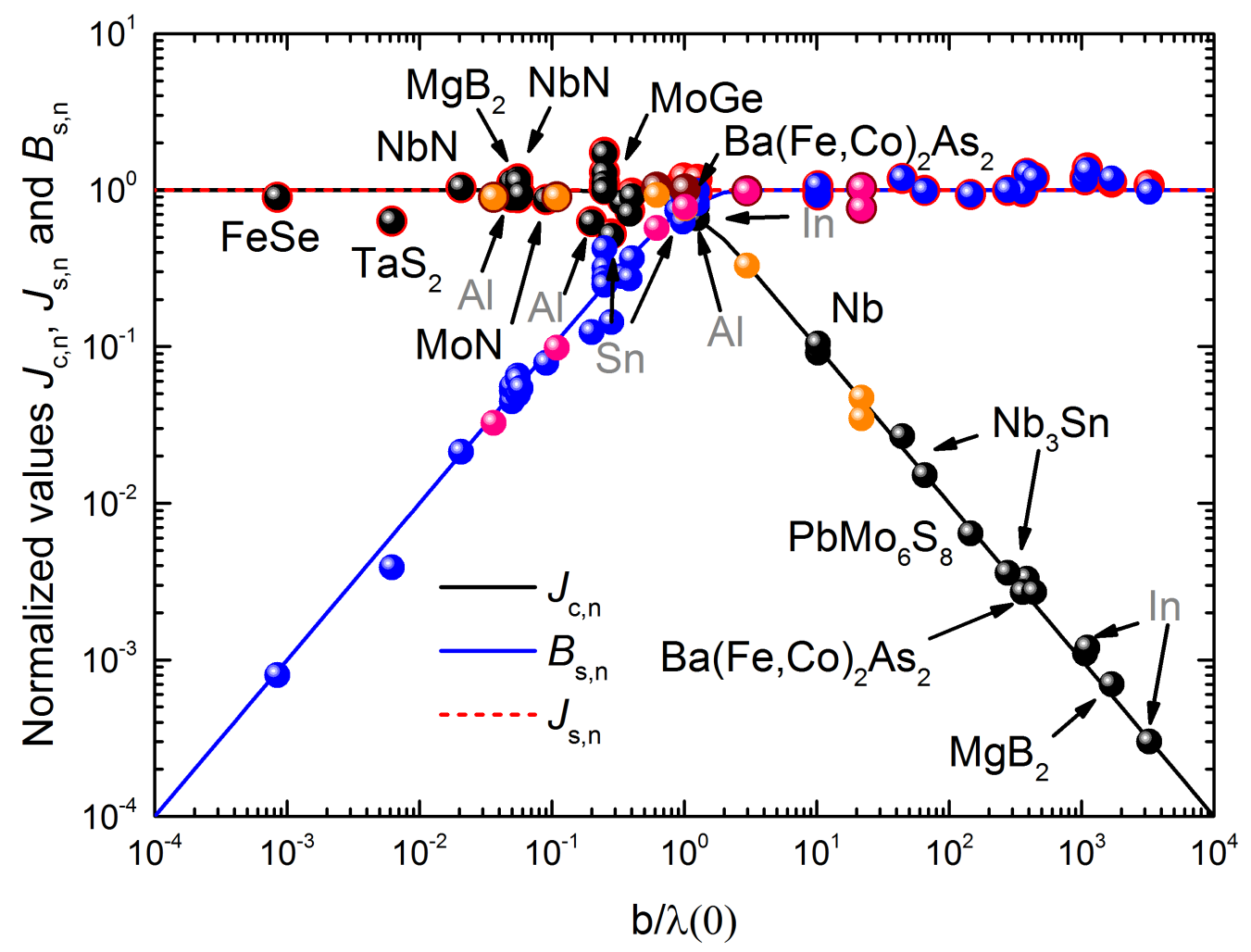

Figure 4.2: Normalized self-field surface current density (red line and red data points), normalized self-field critical current density (black line and black data points) and normalized surface field (blue line and blue data points) vs the sample half-thickness divided by the London penetration depth. The orange data points are the normalized critical current densities for YBCO samples which are plotted against $b / \lambda_{c}$. The pink data points are the normalized surface fields times the anisotropy against $b / \lambda_{c}$ for $\mathrm{YBCO}$ again. For the few examples of cylindrical wires the data is plotted versus $a / 2 \lambda$ where $a$ is the wire radius. The grey labels indicate type I superconductors, whilst the black labels indicate type II superconductors. This graph is produced from tables 4.1 and 4.2 and can be found as supplementary figure 4 in [64]. 


\begin{tabular}{|c|c|c|c|c|c|c|c|c|c|}
\hline Material & $2 a(\mathrm{~nm})$ & $2 b(\mathrm{~nm})$ & $\kappa$ & $\lambda(0)(\mathrm{nm})$ & $J_{c}(0)\left(\mathrm{MA} / \mathrm{cm}^{2}\right)$ & $J_{c, n}$ & $J_{s, n}$ & $B_{s, n}$ & $b / \lambda(0)$ \\
\hline \multirow{5}{*}{$\mathrm{Al}$ (film) } & 610 & 89 & $0.03[31]$ & $50 \pm 10$ 69] & $4.44[70]$ & 0.8287 & 1.0368 & 0.7375 & 0.89 \\
\hline & 680 & 98 & & 46-51 711 & 3.38 70] & 0.6386 & 0.831 & 0.6258 & 0.98 \\
\hline & 880 & 99 & & $51.5 \square 72]$ & 4.06 [70] & 0.9135 & 1.1941 & 0.9044 & 0.99 \\
\hline & 500 & 34 & & & 3.82 70] & 0.8274 & 0.859 & 0.2813 & 0.34 \\
\hline & 300 & 20 & & & 3.68 70] & 0.6198 & 0.628 & 0.124 & 0.2 \\
\hline \multirow[t]{3}{*}{$\mathrm{Al}$ (nano wire) } & 10 & 5 & & & $9.23[73$ & 1.0391 & 1.04 & 0.052 & 0.05 \\
\hline & 8.4 & 5 & & & $7.94[73]$ & 0.8937 & 0.8944 & 0.0447 & 0.05 \\
\hline & 5.4 & 5 & & & $4.94 \quad 73]$ & 1.1115 & 1.1124 & 0.0556 & 0.05 \\
\hline \multirow[t]{6}{*}{ In (thin-film) } & 360 & 100 & $0.11[31]$ & $40[31]$ & 41.1 [74] & 0.7892 & 1.163 & 0.9865 & 1.25 \\
\hline & 320 & 100 & & & $2 0 . 9 \longdiv { 7 4 ] }$ & 0.6566 & 0.9676 & 0.8208 & 1.25 \\
\hline & 520000 & & & & 0.0105 75] & 0.0003 & 1.072 & 0.975 & 3250 \\
\hline & 270000 & & & & 0.021175 & 0.0007 & 1.119 & 1.1813 & 1687.5 \\
\hline & 170000 & & & & $0.036 \sqrt{75 !}$ & 0.0011 & 1.202 & 1.1688 & 1062.5 \\
\hline & 170000 & & & & 0.0347 75] & 0.0011 & 1.158 & 1.1688 & 1062.5 \\
\hline \multirow{2}{*}{$\begin{array}{l}\text { Sn (nanowire) } \\
\text { (film) }\end{array}$} & 70 & & $0.23[31]$ & 56-68 [76] & $9.1[77]$ & 0.5091 & 0.5226 & 0.1437 & 0.2823 \\
\hline & 1900 & 50 & & & $16.2[78]$ & 0.9065 & 0.9551 & 0.3655 & 0.4032 \\
\hline \multirow[t]{2}{*}{$\mathrm{Nb}$ (thin film) } & 82000 & 1000 & 1 [79] & $47 \pm 5801,41 \pm 4$ 81] & 5.12 [82] & 0.091 & 0.928 & 0.9282 & 10.2 \\
\hline & 49000 & 1000 & & $59.4 \pm 0.283$ & 5.9282 & 0.1043 & 1.064 & 1.0639 & 10.2 \\
\hline $\mathrm{TaS}_{2}$ (thin film) & 450 & 4.2 & $\begin{array}{l}12.6-13.6[84] \\
9.8[86], 9.5[87]\end{array}$ & $\begin{array}{l}260-302[84] \\
613[87], 410[88]\end{array}$ & $0.62[85]$ & 0.631 & 0.631 & 0.0039 & 0.00621 \\
\hline \multirow{5}{*}{$\begin{array}{l}\mathrm{Nb}_{3} \mathrm{Sn} \text { (Wire) } \\
\text { (Commercial Tape) } \\
\text { (Thin film) }\end{array}$} & 101000 & & $22[31]$ & $65[31]$ & 0.56 [89] & 0.0033 & 1.27 & 1.2819 & 388.46 \\
\hline & 94000 & & & & $0.46[89]$ & 0.0027 & 0.972 & 0.9762 & 361.54 \\
\hline & 150000 & 36000 & & & 0.761 89] & 0.0036 & 0.992 & 0.9969 & 276.92 \\
\hline & 13000000 & 8500 & & & $2.59 \square$ & 0.0151 & 0.9904 & 0.9872 & 65.38 \\
\hline & 13000000 & 5760 & & & $4.58[901$ & 0.0268 & 1.186 & 1.1875 & 44.31 \\
\hline \multirow[t]{4}{*}{$\mathrm{MgB}_{2}$ (thin film) } & 320 & 10 & $26[91]$ & $85[92]$ & 121 93] & 1.1698 & 1.171 & 0.065 & 0.05556 \\
\hline & 5000 & & & $100[94]$ & $78.2[95]$ & 1.1179 & 1.119 & 0.0621 & 0.05556 \\
\hline & & 100 & & & 84.1 [96] & 0.8881 & 0.889 & 0.0493 & 0.05556 \\
\hline & 1200000 & 200000 & & & 0.085 [97] & 0.0012 & 1.376 & 1.3333 & 1111.11 \\
\hline \multirow[t]{3}{*}{$\mathrm{NbN}$ (thin film) } & 8900 & 8 & $40[31]$ & 200 [31] & 7.97 .9 [98] & 1.0349 & 1.035 & 0.0213 & 0.0206 \\
\hline & 300 & 8 & & 194 99] & 14.37 .9 [98] & 1.0349 & 1.035 & 0.0213 & 0.0206 \\
\hline & 6000 & 22.5 & & & $7.47 \lcm{1001}$ & 0.9338 & 0.9348 & 0.0542 & 0.058 \\
\hline MoN (wire) & 160 & & $54[101]$ & $440 \pm 40[102]$ & 0.5923 [103] & 0.8652 & 0.8676 & 0.0786 & 0.0909 \\
\hline FeSe (Single Atomic Layer) & 1500000 & 0.55 & $72[104]$ & $324[104]$ & $1.65[55]$ & 0.897 & 0.897 & 0.0008 & 0.000849 \\
\hline \multirow[t]{5}{*}{ MoGe (thin film) } & 10000 & 200 & $94 \pm 14$ [1051, [106] & $4 0 0 \longdiv { 1 0 7 ] }$ & $1.75 \lcm{105]}$ & 1.6958 & 1.731 & 0.424 & 0.25 \\
\hline & 20000 & 200 & & & 1.31 105] & 1.2697 & 1.296 & 0.3174 & 0.25 \\
\hline & 25000 & 200 & & & $1.13 \lcm{105]}$ & 1.0953 & 1.118 & 0.2738 & 0.25 \\
\hline & 30000 & 200 & & & $1.04 \square$ & 1.0081 & 1.029 & 0.252 & 0.25 \\
\hline & 40000 & 200 & & & $1.02 \quad 105$ & 0.9885 & 1.009 & 0.2471 & 0.25 \\
\hline $\mathrm{Ba}(\mathrm{Fe}, \mathrm{Co})_{2} \mathrm{As}_{2}$ (wire) & 500000 & & $90[108]$ & $270[108]$ & $\begin{array}{l}0.0078 \text { [109] } \\
\end{array}$ & 0.0027 & 1.201 & 1.1884 & 440.14 \\
\hline (thin film) & 6700 & 220 & & 274 1101, 307 [111] & 2.19 [112] & 0.7032 & 0.738 & 0.2723 & 0.3873 \\
\hline $\mathrm{PbMo}_{6} \mathrm{~S}_{8}$ (wire) & 160000 & & $125[113]$ & $275[113]$ & 0.0216 [114] & 0.0064 & 0.936 & 0.9309 & 145.45 \\
\hline
\end{tabular}

Table 4.1: A table of $J_{c, n}, J_{s, n}$ and $B_{s, n}$ calculated for superconductors of many different sizes and types. The self-field critical current data sets used are referenced next to the $J_{c}(0)$ value for each sample, and the literature Ginzburg-Landau parameter $\kappa$ and penetration depth $\lambda(0)$ are also listed. For samples of cylindrical geometry only the diameter $(2 a)$ is listed and the value $b / \lambda$ is now $a / 2 \lambda$ instead. This is reproduced from table 1 in [64] with Sn added in. 


\begin{tabular}{|l|l|l|l|l|l|l|l|l|l|}
\hline Material & $2 a(\mathrm{~nm})$ & $2 b(\mathrm{~nm})$ & $\kappa$ & $\lambda(0)(\mathrm{nm})$ & $J_{c}(0)\left(\mathrm{MA} / \mathrm{cm}^{2}\right)$ & $J_{c, n}$ & $J_{s, n}$ & $B_{s, n}$ & $b / \lambda_{c}(0)$ \\
\hline YBCO (thin film) & 500000 & 850 & $95[31]$ & $125[115]$ & $31.8[60,[65]$ & 0.9251 & 1.04 & 1.037 & 0.6182 \\
& 500000 & 1400 & & & $26[116,[117$ & 0.7637 & 1.011 & 1.011 & 1.0182 \\
& 50000 & 50 & & & $30[118$ & 0.8966 & 0.897 & 0.177 & 0.0364 \\
& 5000 & 150 & & & $28.9[119$ & 0.8984 & 0.902 & 0.484 & 0.1091 \\
(single crystal) & 2000000 & 30000 & & & $2.04[120]$ & 0.0473 & 1.032 & 1.032 & 21.8182 \\
& 2000000 & 30000 & & & $1.5[120$ & 0.0349 & 0.761 & 0.761 & 21.8182 \\
(STI tape) & 500000 & 4500 & & $135(22 \mathrm{~K})$ & $10.6[117$ & 0.3279 & 0.984 & 0.984 & 2.9861 \\
\hline
\end{tabular}

Table 4.2: A table of $J_{c, n}, J_{s, n}$ and $B_{s, n}$ calculated for different samples of the anisotropic YBCO cuprate superconductor. The self-field critical current data sets used are referenced next to the $J_{c}(0)$ value for each sample, and the literature Ginzburg-Landau parameter $\kappa$ and penetration depth $\lambda(0)$ are also listed. This is reproduced from table 1 in [64]. 


\section{Chapter 5}

\section{Temperature dependence of self-field critical current and penetration depth}

In chapter 4 it was shown that the ground-state self-field critical current density follows the generic relationship

$J_{c}(s f, T)=\frac{B_{c}}{\mu_{0} \lambda} g(a, b, \lambda) \quad$ (Type I), $\quad J_{c}(s f, T)=\frac{B_{c 1}}{\mu_{0} \lambda} g(a, b, \lambda) \quad$ (Type II)

with the geometric factor of

$$
g(a, b, \lambda)=\frac{\lambda}{b} \tanh \frac{b}{\lambda}
$$

for thin film rectangular samples and $g(a, b, \lambda)=\frac{\lambda}{2 a} \tanh \frac{2 a}{\lambda}$ for cylindrical wires. This relationship was however only shown for the ground state ( $T$ $=0 \mathrm{~K})$. A question can be asked as to whether this relation is sustained as a function of temperature? 


\subsection{Tin - high aspect ratio}

First we will look at the example of Sn. Experiments by Schawlow et al [121] were only able to measure $\Delta \lambda(T)=\lambda(T)-\lambda(0)$. However they used a two-fluid model to estimate a ground-state penetration depth value of $\lambda(0)=52 \mathrm{~nm}$, which is close to other measured values of $\lambda(0)$ such as 52 $\mathrm{nm}$ [122], $49 \mathrm{~nm}$ [123] and $50 \mathrm{~nm}$ [124].

The directly measured normalized $\lambda(T)$ data [121] is plotted in figure 5.1 (blue points). Also plotted here is $\lambda(T)$ calculated from a $J_{c}(s f, T)$ dataset from Hunt [78] using equation 5.1 (red points). The film here has a high aspect ratio with the width being $1.9 \mu \mathrm{m}$ and the thickness being $50 \mathrm{~nm}$. The thickness is near the penetration depth and so in the scaling graph from chapter 4 the data would sit near the knee. The solid curve is the theoretical BCS calculation [125].

The temperature dependence of $\lambda(T)$ calculated from the $J_{c}(s f, T)$ data matches remarkably well with the $\lambda(T)$ data measured by Schawlow et al as well as with the theoretical BCS temperature dependence. The exponentially flat temperature dependence of $\Delta \lambda$ at low temperature is evident in all cases and is characteristic of $s$-wave superconductivity.

\subsection{Tin - small aspect ratio}

The $J_{c}(s f, T)$ data measured by Hunt [78] was for a thin film with a high aspect ratio allowing us to ignore the width. A range of thin film Sn samples that have aspect ratios closer to 1 have been measured by Song et al [126], [127]. They found that there was a crossover in the $J_{c}(s f, T)$ temperature dependence as the thickness changed.

To examine this data we now use an approximation for the geometric factor $g(a, b, \lambda)$ more appropriate to equiaxed samples where $b$ in equation 5.1 is replaced by $b^{*}=a b /(a+b)$. The theoretical background of this is explored further in chapter 9 . For the Song data there are 4 samples where 


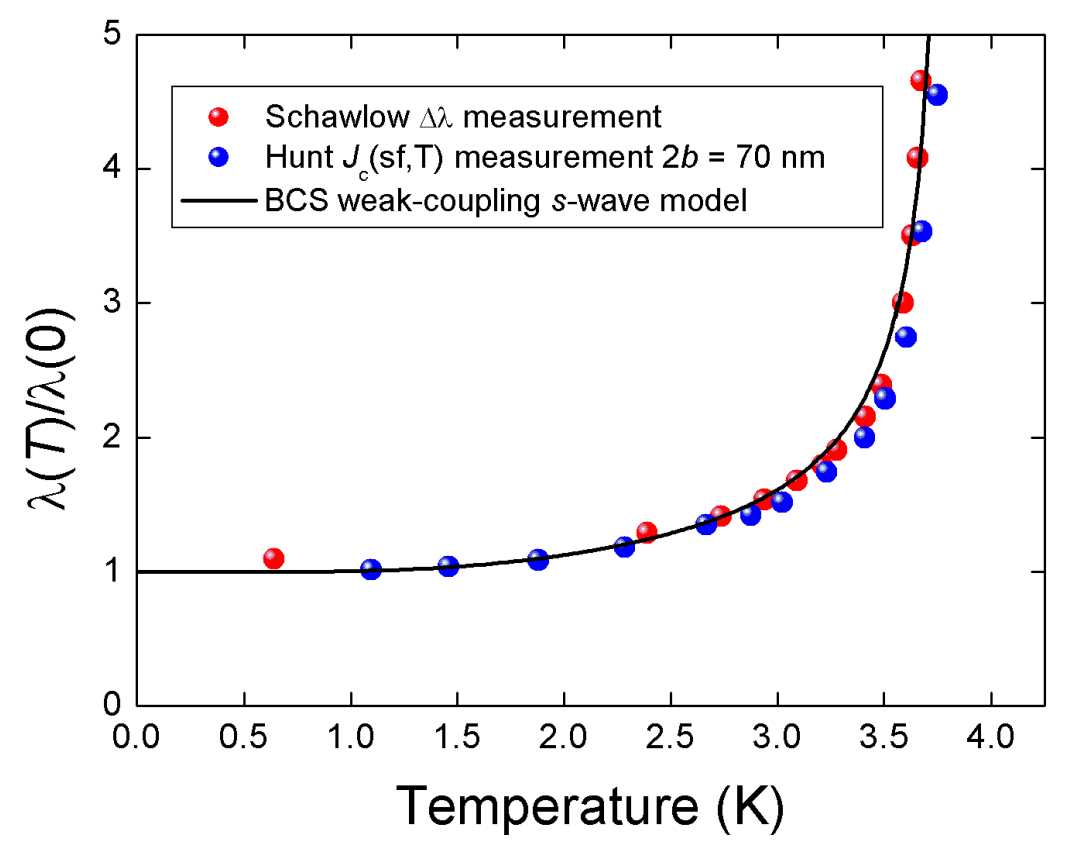

Figure 5.1: Comparison of the temperature dependence of normalised $\lambda(T)$ calculated from $J_{c}(s f, T)$ with independent measurements of $\lambda(T)$ for Sn. The blue $J_{c}(s f, T)$-derived data is from [78] and has been converted into $\lambda(T)$ data using equation 5.1. The red $\lambda(T)$ data is from [121]. The black line is calculated using the BCS theory [125].

$b^{*}$ crosses from $b^{*}>1$ to $b^{*}<1$.

Plotted in figure 5.2 is the normalized $\lambda(T)$ derived from the $J_{c}(s f, T)$ data of Song [126], [127] along with the previous directly measured normalized $\lambda(T)$ data [121] and the theoretical BCS calculation [125].

It can be seen that despite the crossover in temperature dependence that occurs when $b^{*} \simeq \lambda$, the derived $\lambda(T)$ matches well with the independently measured data, confirming that equation 5.1 along with the modified $b^{*}$ in 5.2 describes the temperature dependence of $J_{c}(s f, T)$ quite well. The only significant differences here are largely attributable to the absolute 


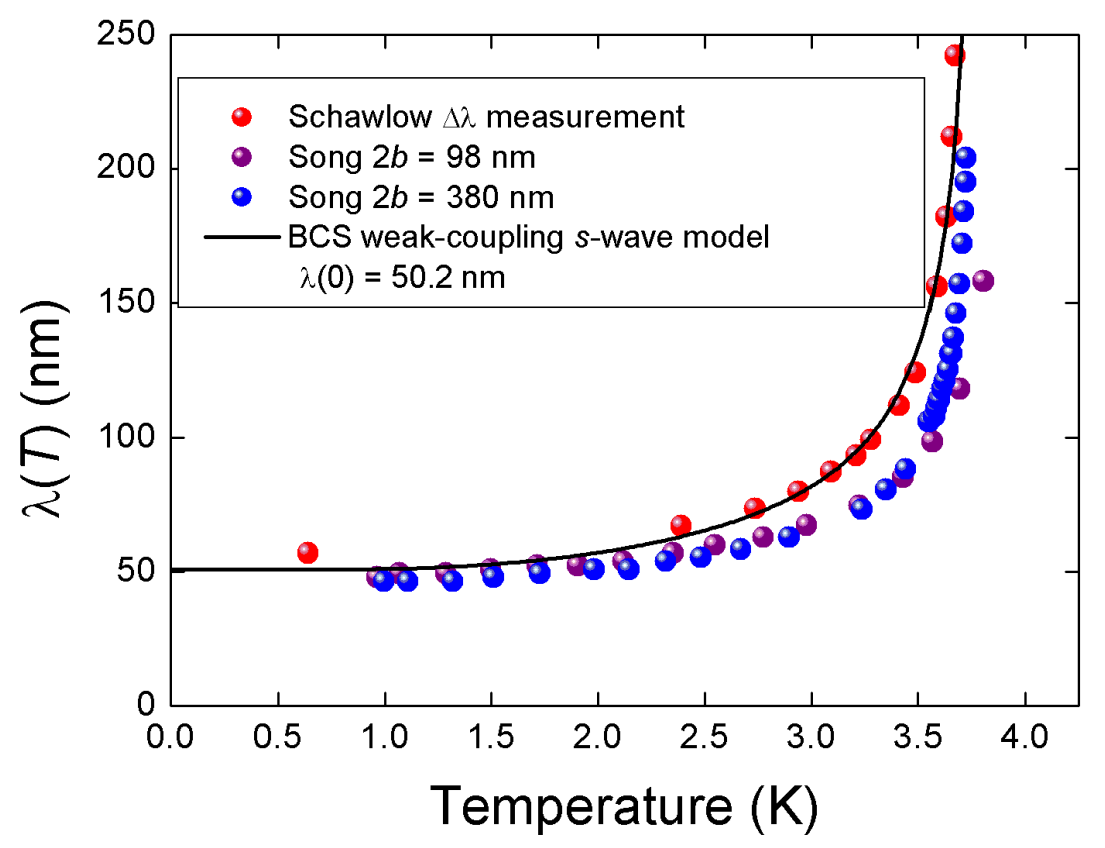

Figure 5.2: Comparison of the temperature dependence of normalised $\lambda(T)$ calculated from $J_{c}(s f, T)$ with independent measurements of $\lambda(T)$ of $\mathrm{Sn}$. The blue and purple $J_{c}(s f, T)$-derived data is from [126], [127] and has been converted into $\lambda(T)$ data using equation 5.1. The red $\lambda(T)$ data is from [121]. The black line is calculated using the BCS theory [125].

value and the evidently higher $T_{c}$ in the Song samples.

\section{3 $\mathrm{BaKBiO}_{3}$}

An example of a type II superconductor $\mathrm{Ba}_{0.6} \mathrm{~K}_{0.4} \mathrm{BiO}_{3}$ is shown in figure 5.3. Here the $J_{c}(s f, T)$ data from [128] is compared with $B_{c 1}$ data measured by [129].

The comparison is made as follows. The $J_{c}(s f, T)$ dataset is used to calculate $\lambda(T)$ using equation 5.1 from which $B_{c 1}$ is then calculated from 


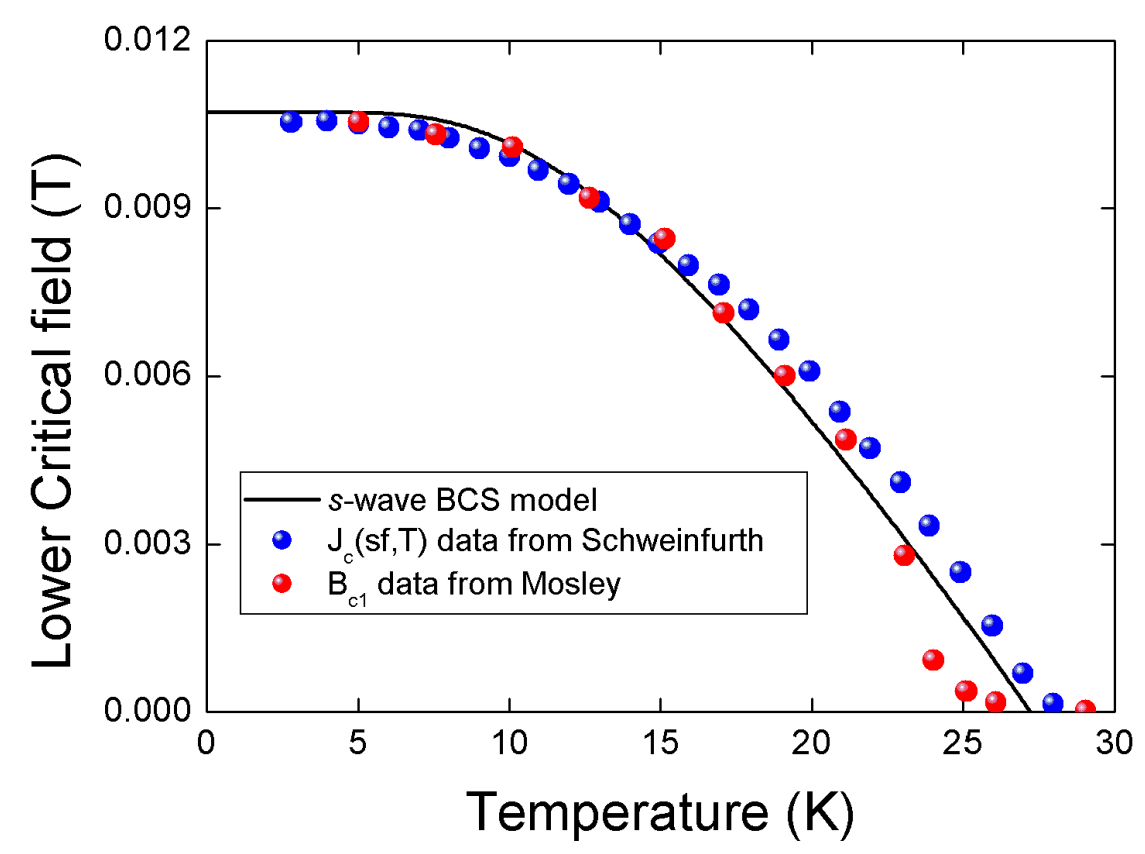

Figure 5.3: Comparison of the temperature dependence of $B_{c 1}$ derived from $J_{c}(s f, T)$ data (red) [128] with independently measured $B_{c 1}$ data (blue) from [129] for $\mathrm{BaKBiO}_{3}$. For comparison the theoretical BCS result is plotted using $\lambda(0)=270 \mathrm{~nm}$.

equation 2.10. The value of the Ginzburg-Landau parameter was taking to be $\kappa=70$ [130]. The film thickness was $150 \mathrm{~nm}$ [128]. The $B_{c 1}$ values calculated in this way are compared in the figure with direct measurements of $B_{c 1}$ [129].

The resulting plot shows that the temperature dependence of the two data sets matches well, apart from the region near $T_{c}$ where differences can largely be attributed to differences in $T_{c}$. The superconducting properties near $T_{c}$ can be highly affected by the exact composition and crystallinity and the sample reported by Mosley [129] is clearly more inhomogeneous than the sample used by Schweinfurth for critical current measurements 
[128].

An example of the temperature dependence for $d$-wave superconductivity is given previously for $\mathrm{YBa}_{2} \mathrm{Cu}_{3} \mathrm{O}_{y}$ [65] (see figure 3(a)). In contrast to the $s$-wave case the low-temperature behaviour of $\Delta \lambda(T)$ is linear in temperature. The absolute values of $\lambda(T)$ determined from $J_{c}(s f, T)$ were shown to be in excellent agreement with the independently measured $\lambda(T)$ by the group of Hardy et al [131].

In conclusion the temperature dependence of self-field critical current density data when analysed using equation 5.1 gives $\lambda(T)$ values which match both the theoretical BCS behaviour and the independent direct measurements of $\lambda(T)$. This was shown by comparing with measurements of $\Delta \lambda(T)$ in the case of $\mathrm{Sn}$ and measurements of $B_{c 1}$ in the case of $\mathrm{Ba}_{0.6} \mathrm{~K}_{0.4} \mathrm{BiO}_{3}$.

The number of other data sets for which direct comparisons can be made is rather limited. More importantly in some cases the behaviour is beyond the weak coupling limit. In fact the $\mathrm{T}$ dependence can be used as a probe of the coupling strength and the magnitude of the order parameter. This is addressed in the next chapter. 


\section{Chapter 6}

\section{Deriving thermodynamic parameters from self-field critical current}

In the previous chapter it was found that the self-field critical current was dependent almost exclusively on the penetration depth, $\lambda$. By considering how $\lambda$ depends on the temperature which can be determined from the temperature dependence of the superconducting gap, $\Delta(T)$, the self-field critical current data can be fit to determine the key thermodynamic superconducting parameters. In this chapter we explore these ideas.

A new model for extracting the superconducting gap and London penetration depth from the temperature-dependent self-field critical current, $J_{c}(s f, T)$, is presented and discussed. The model is applied to over 90 data sets taken from the literature. Importantly, the data is not selected but represents an exhaustive list of available data sets. The work presented has been published in [132]. 


\subsection{Model for temperature dependent self-field critical currents}

In the previous chapter it was found that the global self-field critical current density of both rectangular thin films and round wires could be described using

$$
J_{c}(s f, T)=\frac{B_{c}(T)}{\mu_{0} \lambda(T)}
$$

for type I superconductors and

$$
J_{c}(s f, T)=\frac{B_{c 1}(T)}{\mu_{0} \lambda(T)}
$$

for type II superconductors where $\mu_{0}$ is the permeability of free space. For samples of dimension large compared with $\lambda$ these equations are multiplied by a geometric factor which is $\frac{\lambda}{b} \tanh \left(\frac{b}{\lambda}\right)$ for rectangular thin films $(b$ is the half thickness) and $\frac{2 \lambda}{b} \frac{I_{1}(b / \lambda)}{I_{0}(b / \lambda)}$ for round wires ( $b$ is the radius). In the case of rectangular thin films where the aspect ratio, $b / a$, is not large the geometric factor can be replaced by a size-dependent factor $\frac{\lambda}{a} \tanh \left(\frac{a}{\lambda}\right)+$ $\frac{\lambda}{b} \tanh \left(\frac{b}{\lambda}\right)$ where $a$ is the half width. This geometric factor is introduced because it is expected that the geometric factor should take a form similar to that for a wire when the sample is reduced to a square cross-section. It is noted that the original paper [132] used this geometric factor however it has recently been found that it should probably take the form $\frac{\lambda}{b^{*}} \tanh \left(\frac{b^{*}}{\lambda}\right)$ where $b^{*}=\frac{a b}{a+b}$. This is discussed in chapter 9 .

By using the expressions for both $B_{c}$ and $B_{c 1}$ (see equation 10 from the background chapter) this can be seen as a direct relation between $J_{c}(s f, T)$ and $\lambda(T)$ where the Ginzburg-Landau parameter $\kappa$ can be taken as a constant, especially for the type II case where it is under a logarithm. Now all is needed is an expression for the $\lambda(T)$ to be able to fit temperaturedependent self-field critical current data. 


\subsubsection{Semiclassical BCS expression for the superfluid den- sity}

An expression for the normalized superfluid density $\rho_{s}(T)=(\lambda(0) / \lambda(T))^{2}$ can be derived using the semi classical approach outlined in [32]. The London approximation $\mathbf{J}=-\mathbb{R} \mathbf{A}$ where $\mathbf{J}$ is the current density and $\mathbf{A}$ is the vector potential, contains the symmetric response tensor $\mathbb{R}_{i i}(T)=$ $1 / \mu_{0} \lambda_{i i}(T)^{2}$ which is proportional to the superfluid density. The form of the symmetric tensor is

$$
\mathbb{R}_{i j}=\frac{e^{2}}{4 \pi^{3} \hbar c} \oint_{\text {FermiSurf }} d S_{k}\left[\frac{v_{F}^{i} v_{F}^{j}}{\left|v_{f}\right|}\left(1+2 \int_{\Delta(k)}^{\infty} \frac{\partial f(E)}{\partial E} \frac{N(E)}{N(0)} d E\right)\right]
$$

where $v_{F}^{i}$ is the Fermi velocity in the direction $i, N(E)$ is the density of states at the energy $E=\sqrt{\varepsilon^{2}+\Delta(\mathbf{k}, T)}, f(E)$ is the Fermi function and $\Delta(\mathbf{k}, T)$ is the superconducting gap. The normalized superfluid density can then be calculated as $\rho_{s}(T)=\mathbb{R}_{i i}(T) / \mathbb{R}_{i i}(0)$.

Expressions can be derived for the two common cases of gap symmetry. For an s-wave superconductor with a spherical Fermi surface the integral can be simplified giving the superfluid density as

$$
\rho_{s}(T)=1-\frac{1}{2 \pi k_{B} T} \int_{0}^{\infty} \cosh ^{-2}\left(\frac{\sqrt{\varepsilon^{2}+\Delta^{2}(T)}}{2 k_{B} T}\right) d \varepsilon
$$

Also for a $d$-wave superconductor with a cylindrical Fermi surface the superfluid density can be simplified to be

$$
\rho_{s}(T)=1-\frac{1}{2 \pi k_{B} T} \int_{0}^{2 \pi} \cos ^{2}(\varphi) \int_{0}^{\infty} \cosh ^{-2}\left(\frac{\sqrt{\varepsilon^{2}+\Delta^{2}(T, \varphi)}}{2 k_{B} T}\right) d \varepsilon d \varphi .
$$

In the $d$-wave case the gap is expressed as $\Delta(T, \varphi)=\Delta(T) \cos (2 \varphi)$ where the angular part is due to the variation of the gap around the Fermi surface. 


\subsubsection{Energy gap temperature dependence}

In order to calculate the superfluid density the energy gap, $\Delta(T)$, must be determined first. In general $\Delta(T)$ must be calculated self-consistently using BCS theory or the more complicated Eliashberg theory [133]. Here we use a phenomenological gap equation found in [134] and it takes the form

$$
\Delta(T)=\Delta(0) \tanh \left(\frac{\pi k_{b} T_{c}}{\Delta(0)} \sqrt{\eta \frac{\Delta C}{C}\left[\frac{T_{c}}{T}-1\right]}\right)
$$

where $\eta$ is a constant that depends on the pairing symmetry $(2 / 3$ for $s-$ wave and $7 / 5$ for $d$-wave) and $\Delta C / C$ is the specific heat jump at $T_{c}$. The alpha-Padamsee model [135] can be used to derive the $d$-wave $\eta$ value. This solution is able to replicate the gap temperature dependence for both weak- and strong-coupling superconductors.

With these equations we can now rather generally fit $J_{c}(s f, T)$ for superconductors with the geometry of a round wire or rectangular thin film. The superconductor type can be either type I or type II and the pairing symmetry can be $s$-wave or $d$-wave.

For a reliable fit the $J_{c}(s f, T)$ data must have enough data points that span much of the temperature range and reach close enough to $T=0 \mathrm{~K}$. This is necessary to be able to reliably determine $\Delta(0)$ and $\lambda(0)$. Another important point is that the sample must have minimal weak-links as these reduce the magnitude of the current, changing the value of $\lambda(0)$ resulting from the analysis. As stated at the beginning of chapter 3, much of the data is has been digitized from the literature. This has been referenced in the tables at the end of the chapter.

\section{$6.2 s$-wave superconductors}

First we show the results from fitting $s$-wave datasets using equations 6.1 . 6.2. 6.4 and 6.6. The references for the datasets fitted here can be found in 
[132] using the references from table 3 in the appendix of that publication which is also reproduced here. Nine different superconductors ( $\mathrm{Al}$, In, $\mathrm{Sn}, \mathrm{NbN}, \mathrm{MoGe}, \mathrm{Ba}_{0.6} \mathrm{~K}_{0.4} \mathrm{BiO}_{3}, \mathrm{HoNi}_{2} \mathrm{~B}_{2} \mathrm{C}, \mathrm{YNi}_{2} \mathrm{~B}_{2} \mathrm{C}, \mathrm{H}_{2} \mathrm{~S}$ (155 GPa)) with their derived fitting parameters are shown in table 6.1 along with the references of the datasets. Eight of the superconductors are shown with the raw $J_{c}(s f, T)$ data and fitted curve in figure 6.1 which is reproduced from figure 2 in [132].

The red curves and data points are the calculated values of $\lambda(T)$ using the $J_{c}(s f, T)$ raw data and fitted curve which are blue. For this calculation equations 6.1 for type I and 6.2 for type II are used. The green data point with its error bars shows the literature value of $\lambda(0)$ together with its uncertainty. It can be seen that the fits give $\lambda(0)$ values close to the literature value in most cases.

It should be noted here however that an absolute experimental determination of $\lambda(0)$ is in general quite difficult and using $B_{c 1}$ measurements to determine it has its problems [65]. This is because $B_{c 1}$ is determined by looking at magnetisation data to find the point of non-linear behaviour. $B_{c 1}(0)$ is then found by extrapotating to $T=0 \mathrm{~K}$, however a true extrapolation of $B_{c 1}$ needs information of the gap function, which is not usually known or used.

An example where usage of $B_{c 1}$ to find the penetration depth lead to an incorrect result is the case of Ren et al [136], who found from their measurements of the penetration depth that the pnictide superconductor $\mathrm{Ba}_{x} \mathrm{~K}_{1-x} \mathrm{Fe}_{2} \mathrm{As}_{2}$ did not sit on the Uemura universal curve [137]. However a later measurement by a senior author of the same paper found using optical measurements the agreed upon value of $\lambda(0)$ which put the superconductor back on the Uemura universal curve [138].

For the superconductors where multiple data sets were available only a representative fit is shown as is the case for $\mathrm{In}, \mathrm{Sn}, \mathrm{NbN}$ and MoGe. The derived parameters for all the datasets fitted can be found in table 6.1. The ability to estimate values of $\lambda(0)$ that are in good agreement with literature 


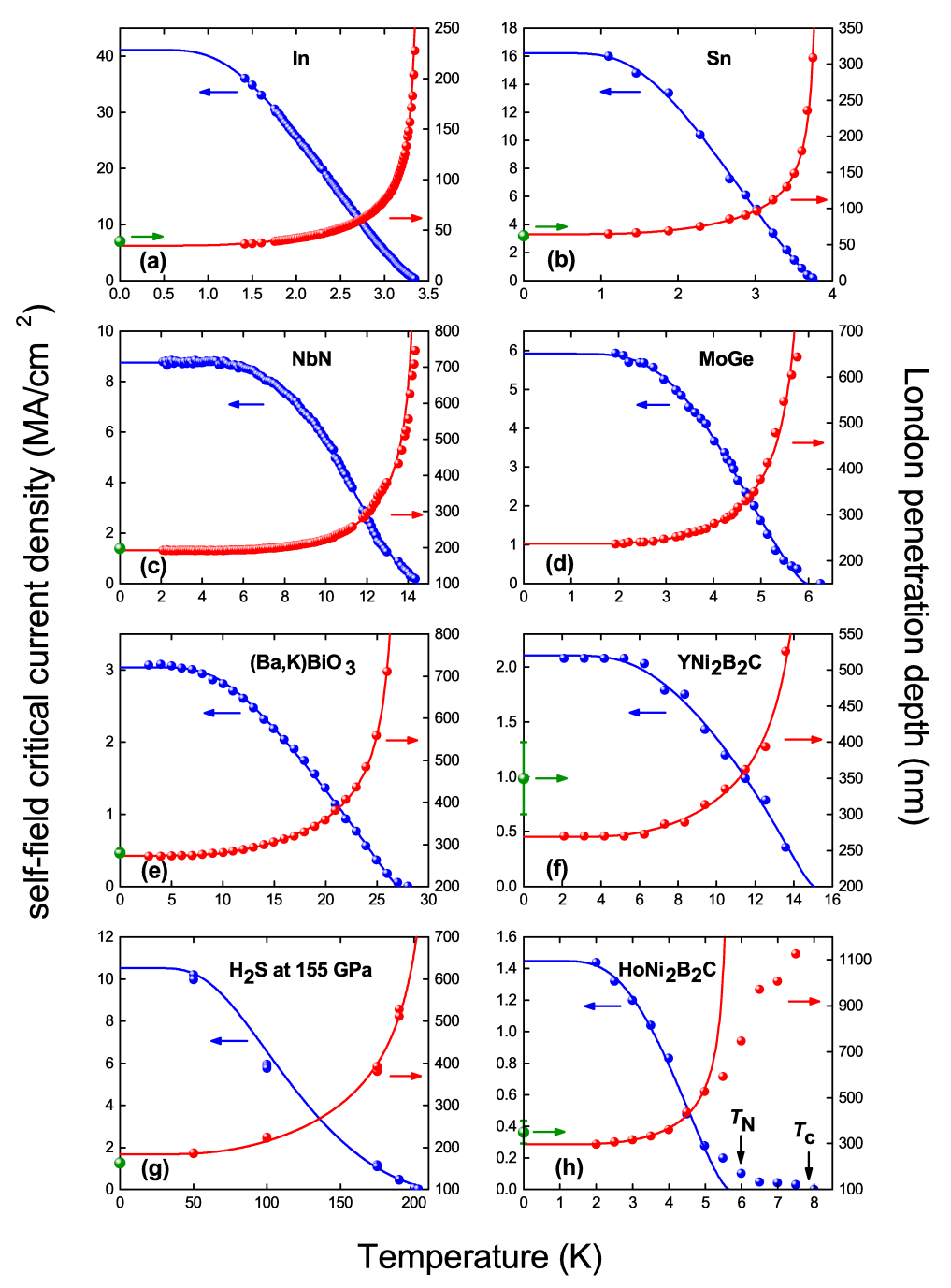

Figure 6.1: Reproduced from figure 2 in [132]. Experimental self-field $J_{c}(T)$ data for $s$-wave superconductors as annotated (left axis, blue) together with values of $\lambda$ (right axis, red) derived from equations 6.1, 6.2, 6.4 and 6.6. The solid curves are the fits using the model described. Note the variable offset of the $\lambda(T)$ axis. The single green data points at $T=0$ are, where available, reported ground-state values of $\lambda_{0}$ found in table 6.1. Fit parameters: $\lambda(0), \Delta(0), \Delta C / C$ and $T_{c}$ are also summarised in table 6.1. 
values certainly reinforces the validity of equations 6.1 and 6.2 .

The shape of the fits matches well with the data points in all cases except one $\left(\mathrm{HoNi}_{2} \mathrm{~B}_{2} \mathrm{C}\right)$ which is expanded upon below. The exponentiallyflat temperature dependence of $\lambda(T)$ at low temperature, characteristic of $s$-wave symmetry, can be seen in all of the fits. Also the effect of strong coupling can be seen as the flat region extends to higher relative temperatures and the drop-off is steeper.

Special mention must be made of both $\mathrm{HoNi}_{2} \mathrm{~B}_{2} \mathrm{C}$ and $\mathrm{H}_{2} \mathrm{~S}$ (155 GPa). $\mathrm{HoNi}_{2} \mathrm{~B}_{2} \mathrm{C}$ shows a weak increase in $J_{c}(s f, T)$ down to the Néel temperature whereupon $J_{c}(s f, T)$ rises similar to other superconductors. This is a two-step behaviour of $J_{c}(s f, T)$ indicative of the opening of two distinct gaps, and a similar behaviour is seen in measurements of $\Delta(T)$ [139].

As for $\mathrm{H}_{2} \mathrm{~S}$ compressed at $155 \mathrm{GPa}$ the $J_{c}(s f, T)$ data is based on magnetisation measurements which are discussed in detail here [140]. Compressed $\mathrm{H}_{2} \mathrm{~S}$ is a newly discovered superconductor that has been able to reach the highest ever recorded critical temperature of $203 \mathrm{~K}$ [20] and so is of great interest currently.

This model provides a first estimate of the energy gap in this system (around $27.3 \mathrm{meV}$ ). The result indicates that the system may be a weaklycoupled rather than a strongly-coupled superconductor due to the low BCS ratio (around 3.2) however the amount of data points was very low and so the result must be considered with caution. Due to the extremely high pressures present in this system, conventional methods would make a measurement of the energy-gap quite difficult.

Figure 6.2 compares the values of the ground-state superconducting gap magnitude, $\Delta(0)$, and the specific heat jump at critical temperature, $\frac{\Delta C}{C}$, found by fitting the $J_{c}(s f, T)$ to the extended BCS model presented above (denoted by the word derived), with independently measured values from the literature (denoted by the word measured). The data points for the graph are found in tables 6.1, 6.2 and 6.3 along with the references for the $J_{c}(s f, T)$ datasets that have been fitted and literature values of $\Delta(0)$ 
and $\frac{\Delta C}{C}$.

On the left are plots for s-wave superconductors and on the right are plots for $d$-wave superconductors. Looking in particular at the top left of figure 6.2 it can be seen that the model is good at estimating $\Delta(0)$ values close to what is found in literature for s-wave superconductors.
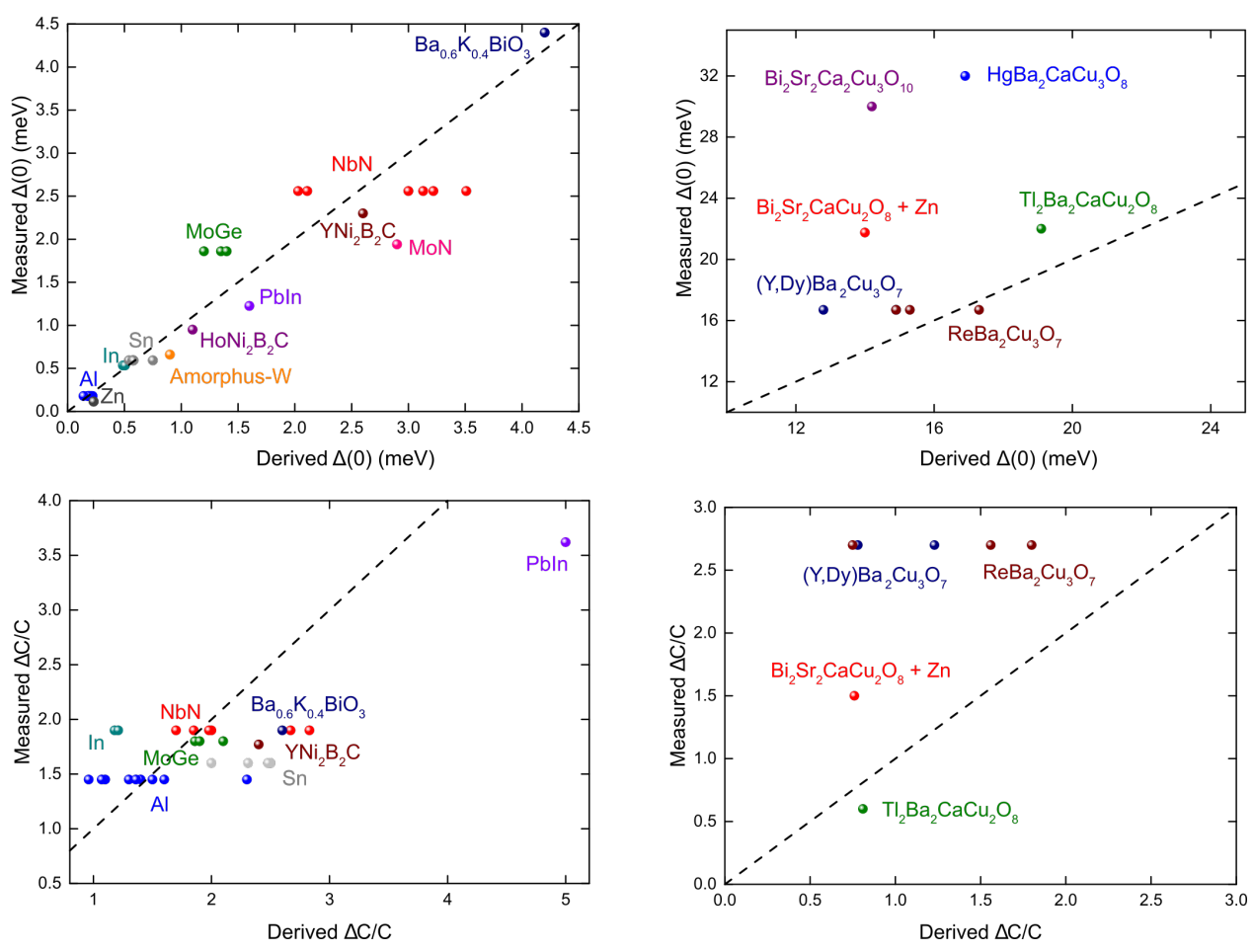

Figure 6.2: Comparison between values of the gap magnitude (top) and specific heat jump (bottom) at $T_{c}$ derived from self-field ciritcal current measurements (derived) vs experimentally measured values (measured). The plots on the left are for s-wave superconductors and the plots on the right are for $d$-wave superconductors.

The specific heat jump at critical temperature, $\frac{\Delta C}{C}$, does not see as good a correlation with independent experimental data as $\Delta(0)$ but it can still be estimated reasonably in some cases. This may be due to the fact that 
$\frac{\Delta C}{C}$ is largely determined by the shape of the $J_{c}(s f, T)$ curve close to $T_{c}$ whilst $\Delta(0)$ is largely determined by where the $J_{c}(s f, T)$ curve begins its downturn with respect to temperature. For measurements close to $T_{c}$, a determination of $I_{c}$ will be less accurate due to noise and inhomogeneities in the sample. A comparison between the dervied $\frac{\Delta C}{C}$ from this study and literature values is also shown in the bottom left of figure 6.2

Another reason for the lesser success in estimating $\frac{\Delta C}{C}$ could be related to the use of a phenomenological model. The model captures the general shape of the $\Delta(T)$ curve but it may not be completely accurate.

This finding however does show that $\Delta(0)$ may be estimated using measurements of $J_{c}(s f, T)$. All that is needed for this estimate is an accurate determination of the shape of the $J_{c}(s f, T)$ curve as $\Delta(0)$ does not depend on the absolute magnitude.

\section{$6.3 d$-wave superconductors}

Table 6.2 shows the parameters dervied from fitting the $J_{c}(s f, T)$ data to the extended BCS model for several $d$-wave cuprate superconductors. It can also be found as table 4 in the appendix of [132] along with references for all the datasets used. The data is fitted using equations 6.1, 6.2, 6.5 and 6.6 .

Figure 6.3 (reproduced from figure 3 in [132]) shows the resulting fits for eight of the nine cuprate datasets. Again the red lines and data points are the calculated $\lambda(T)$ using equation 6.2 and the $J_{c}(s f, T)$ raw data and fitted curves shown in blue. The green data points and error bars show the independent literature values of $\lambda(0)$.

The fitted lines again match well with the datasets and the derived $\lambda(0)$ are very close to those measured in the literature. The low-temperature behaviour is linear as expected for $d$-wave superconductors, contrasting the $s$-wave case.

Shown in the top right of figure 6.2 is a comparison between the values 

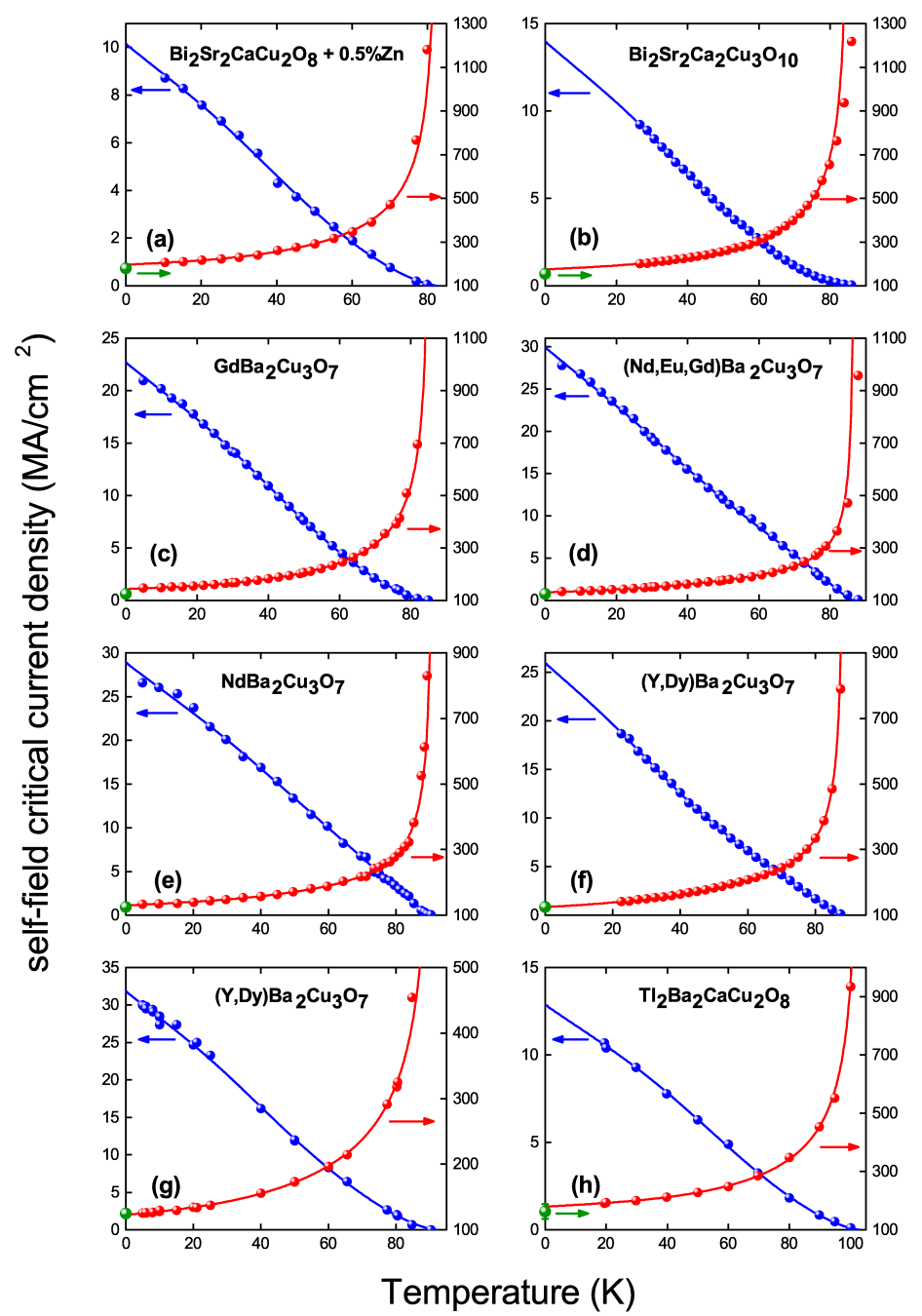

Figure 6.3: Reproduced from figure 3 in [132]. Experimental self-field $J_{c}(T)$ data for $s$-wave superconductors as annotated (left axis, blue) together with values of $\lambda$ (right axis, red) derived from equations 6.2, 6.5 and 6.6. The solid curves are the fits using the model described. Note the variable offset of the $\lambda(T)$ axis. The single green data points at $T=0$ are, where available, reported ground-state values of $\lambda_{0}$ found in table 6.2. Fit parameters: $\lambda(0), \Delta(0), \Delta C / C$ and $T_{c}$ are also summarised in table 6.2 . 
of $\Delta(0)$ derived here and values from literature. The derived $\Delta(0)$ values match well enough for the $\mathrm{RBa}_{2} \mathrm{Cu}_{3} \mathrm{O}_{y}$ samples but not so well for the Bismuth, Mercury and Thallium cuprates. The reason for this is uncertain however we do note that the measurement of the gap in the cuprates is complicated by presence of an addition energy gap known as the pseudogap [141].

The bottom right of figure 6.2 compares the $\frac{\Delta C}{C}$ values and it can be seen that this parameter is not estimated well in the case of cuprates. As this fit parameter is largely determined by the $J_{c}(s f, T)$ data close to $T_{c}$ it is not surprising as the cuprates are well known to have $T_{c}$ suppressed from its mean-field value by fluctuations [142]. Extensive measurements by Loram et al [143] have shown in great detail how the electronic specific heat in the cuprates behaves differently from conventional BCS superconductors. $\Delta C / C$ is suppressed by the presence of the pseudogap. It is also reduced by fluctuations below its mean-field value [142], as is the $T_{c}$ value.

The superconductor $\mathrm{GdBa}_{2} \mathrm{Cu}_{3} \mathrm{O}_{y}$ has a large Curie term in the magnetic susceptibility arising from the $\mathrm{Gd}^{3+}$ ions [144]. This fact may complicate measurements of $\lambda(T)$ that use magnetic techniques, however the results presented here have shown $J_{c}(s f, T) \propto \rho_{s}^{3 / 2}$ and so the extracted $\lambda(T)$ should not be affected by the magnetic properties. This is an important bonus of the technique.

\subsection{Nanowire superconductors}

Table 6.3 and figure 6.4 (table 5 from the appendix and figure 6 from [132])) show the derived parameters and fits for nanowire samples. Again the fitted lines match well with the datasets. The derived $\lambda(0)$ match well for the $\mathrm{Al}, \mathrm{MoN}$ and $\mathrm{YBCO}$ samples but for the $\mathrm{Zn}$ and PbIn samples the derived $\lambda(0)$ values are higher. This may be due to weak-links in the samples.

For the example of $\mathrm{PbIn}$, an examination of the voltage-current curves [145] show that there are 3 different peaks in the $d I / d V$ data. This step- 
wise nature of the voltage-current curves indicate that different regions of the superconductor are transitioning to the normal state at different times. This behaviour is indicative of the presence of weak-links that cause a reduction of the critical current.

In the case of $\mathrm{Zn}$, the original papers [146], [147] show a distinct plateau in the voltage-current curves during the transition. This strange behaviour was described as a dissipative superconducting state. The state seems to be directly affect the critical current causing our derived $\lambda(0)$ to be significantly higher then expected.

The nanowire datasets show a slightly different $T$-dependence in $J_{c}(s f, T)$ due to their small size. This is because the term $\frac{\lambda}{b} \tanh \left(\frac{b}{\lambda}\right)$ changes the $\lambda$ dependence as the size is changed.

One last point to note about this analysis is the likely effect of weaklinks. If these are present, the sample is expected to have a reduced critical current, raising the value of $\lambda(0)$ extracted from the analysis. If the number of weak-links are small with the current having a mostly unimpeded path, the analysis will most likely give a $\lambda(0)$ that is close to the proper value. In any case the analysis should always be able to give an upper limit for $\lambda(0)$.

Also if the number of weak-links is small, the temperature dependence is unlikely to change. However as weak-links start affecting the current path, the temperature dependence is likely to transition to something like that predicted by Ambegaokar and Baratoff [148],[149] where $I_{c}(T)=$ $\frac{\pi \Delta(T)}{2 e R_{n}} \tanh \left(\frac{\Delta(T)}{2 k_{B} T}\right)$ and $R_{n}$ is the normal state resistance. This might be an interesting transition to study in the future.

In summary, the model proposed here has been successful in extracting key thermodynamic parameters by fitting the temperature dependence of the self-field critical current. This method is relatively simple to implement making it an attractive option for estimating thermodynamic parameters compared with much more complex experiments conventionally used to extract these parameters e.g. muon spin relaxation, tunneling mea- 

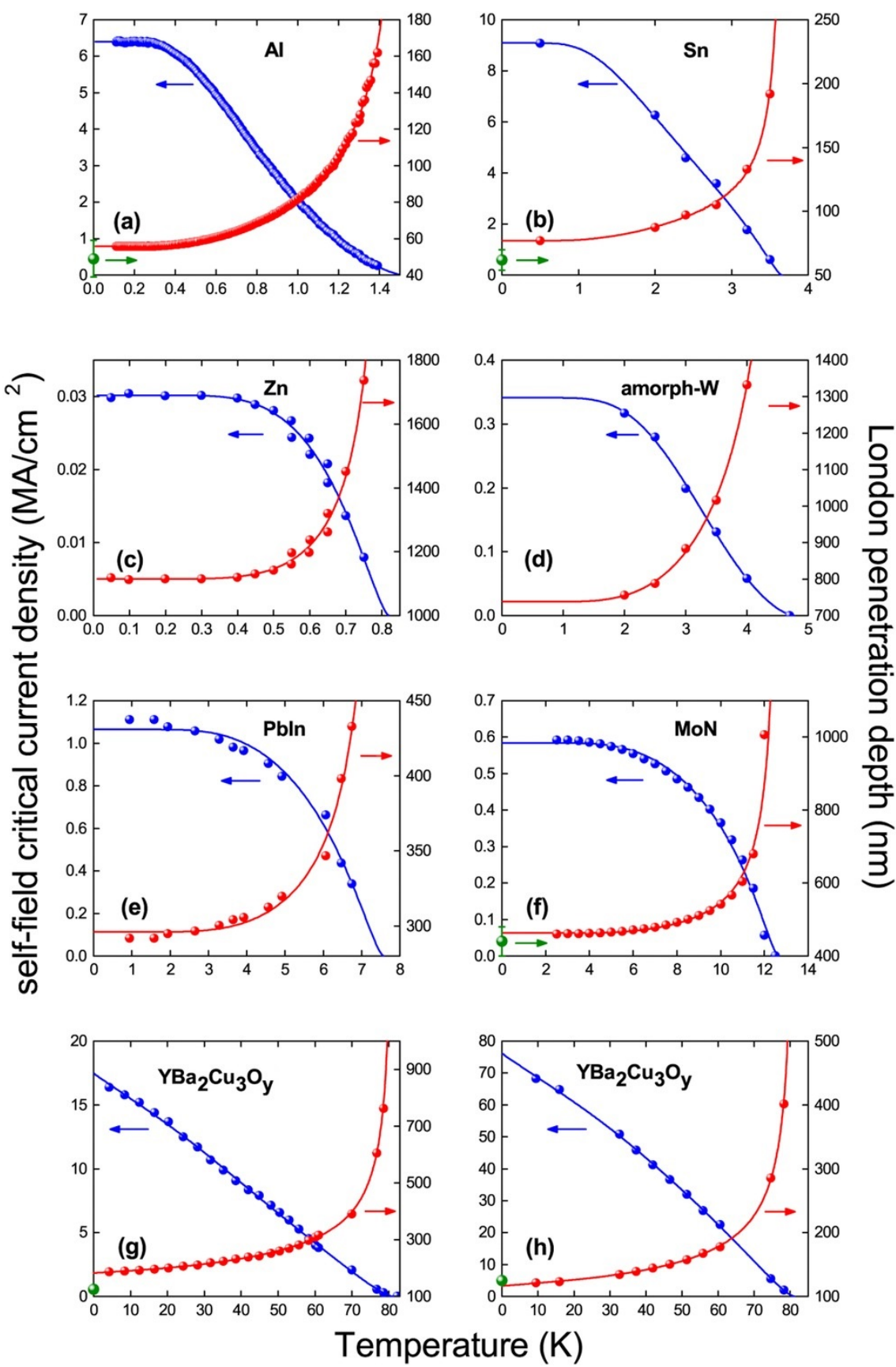

Figure 6.4: Reproduced from figure 6 in [132]. Experimental self-field $J_{c}(T)$ data for various topologies of superconductors as annotated (left axis, blue) together with values of $\lambda$ (right axis, red) derived from equations 6.1 6.2, 6.4 and 6.6. The solid curves are the fits using the model described. Note the variable offset of the $\lambda(T)$ axis. The single green data points at $T=0$ are, where available, reported ground-state values of $\lambda_{0}$ found in table 6.3. Fit parameters: $\lambda(0), \Delta(0), \Delta C / C$ and $T_{c}$ are also summarised in table 6.3 
surements, ARPES and specific heat measurements. Most importantly the analysis confirms our fundamental hypothesis given by equations 6.1 and 6.2 


\begin{tabular}{|c|c|c|c|c|c|c|c|c|c|c|c|}
\hline $\begin{array}{l}\text { Material/ } \\
\text { geometry }\end{array}$ & $\begin{array}{l}2 a \\
\mathrm{~nm}\end{array}$ & $\begin{array}{c}2 b \\
\mathrm{~nm}\end{array}$ & $\kappa$ & $\begin{array}{c}J_{c}(0) \\
\mathrm{MA} / \mathrm{cm}^{2}\end{array}$ & $\begin{array}{l}T_{c} \\
(\mathrm{~K})\end{array}$ & $\begin{array}{c}\begin{array}{c}\Delta_{0} \\
(\mathrm{meV})\end{array} \\
\end{array}$ & $\Delta C / C$ & $\begin{array}{c}\lambda_{0} \\
(\mathrm{~nm})\end{array}$ & $2 \Delta_{0} / k_{B} T_{c}$ & $\begin{array}{l}B_{c 1} \\
(\mathrm{mT})\end{array}$ & $\begin{array}{c}B_{c} \\
(\mathrm{mT})\end{array}$ \\
\hline \multicolumn{12}{|l|}{$s$-wave } \\
\hline \multirow[t]{5}{*}{$\mathrm{Al}$} & 610 & 89 & \multirow[t]{6}{*}{$0.03[31]$} & 4.44 [70] & $1.2 \pm 0.01$ & $0.211 \pm 0.005$ & $1.1 \pm 0.07$ & $49.3 \pm 0.09$ & $4.1 \pm 0.1$ & & 2.9 \\
\hline & 680 & 98 & & 3.38 [70] & $1.18 \pm 0.01$ & $0.22 \pm 0.01$ & $1.6 \pm 0.2$ & $53.8 \pm 0.2$ & $4.3 \pm 0.2$ & & 2.4 \\
\hline & 880 & 99 & & $4.06[70]$ & $1.18 \pm 0.01$ & $0.22 \pm 0.01$ & $1.6 \pm 0.2$ & $49.2 \pm 0.2$ & $4.3 \pm 0.2$ & & 2.9 \\
\hline & 500 & 34 & & 3.82 70] & $1.25 \pm 0.01$ & $0.175 \pm 0.002$ & $1.5 \pm 0.1$ & $55.8 \pm 0.08$ & $3.25 \pm 0.06$ & & 2.2 \\
\hline & 300 & 20 & & 3.68 70] & $1.34 \pm 0.01$ & $0.186 \pm 0.002$ & $1.4 \pm 0.1$ & $59.3 \pm 0.09$ & $3.22 \pm 0.06$ & & 2.0 \\
\hline experiment & & & & & & $0 . 1 7 9 \longdiv { 1 5 0 1 }$ & 1.45 & 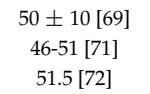 & & & \\
\hline In & 360 & 100 & \multirow[t]{2}{*}{0.11 [31] } & 41.1 [74] & $3.378 \pm 0.002$ & $0.487 \pm 0.003$ & $1.18 \pm 0.01$ & $34.14 \pm 0.06$ & $3.35 \pm 0.01$ & & 22.0 \\
\hline experiment & 320 & 100 & & 20.9774 & $3.489 \pm 0.006$ & $\begin{array}{c}0.505 \pm 0.006 \\
0.525[151] \\
0.541[150]\end{array}$ & $\begin{array}{c}1.21 \pm 0.02 \\
1.9[152]\end{array}$ & $\begin{array}{c}46.4 \pm 0.1 \\
40[31]\end{array}$ & $3.36 \pm 0.01$ & & 11.9 \\
\hline Sn nanowire & 70 & 70 & \multirow[t]{5}{*}{0.23 [31] } & 9.1 [77] & $3.70 \pm 0.04$ & $0.54 \pm 0.05$ & $2.5 \pm 0.6$ & $77.0 \pm 0.3$ & $3.4 \pm 0.3$ & & 9.0 \\
\hline Sn film & 1,900 & 50 & & 16.27 & $3.78 \pm 0.03$ & $0.58 \pm 0.03$ & $2.0 \pm 0.2$ & $64.1 \pm 0.8$ & $3.5 \pm 0.1$ & & 13.0 \\
\hline annular film & 718800 & 170 & & 0.0136 [153] & $3.78 \pm 0.01$ & $0.75 \pm 0.12$ & $2.5 \pm 0.3$ & $41.8 \pm 0.9$ & $4.6 \pm 0.7$ & & 30.7 \\
\hline Sn film & 500 & 98 & & 30.4 126] 127] & $3.89 \pm 0.03$ & $0.57 \pm 0.01$ & $2.31 \pm 0.26$ & $51.7 \pm 0.2$ & $3.4 \pm 0.1$ & & 20.0 \\
\hline $\begin{array}{l}\text { Sn film } \\
\text { experiment }\end{array}$ & 500 & 380 & & $18.3 \quad 126]$ 127] & $3.76 \pm 0.01$ & $\begin{array}{c}0.58 \pm 0.01 \\
0.593[150]\end{array}$ & $\begin{array}{c}2.48 \pm 0.09 \\
1.6[152]\end{array}$ & $\begin{array}{l}46.5 \pm 0.1 \\
56-68 \quad 76]\end{array}$ & $3.59 \pm 0.06$ & & 24.8 \\
\hline \multirow[b]{6}{*}{ experiment } & 8,900 & 8 & \multirow[t]{6}{*}{40 [31] } & 7.9 98] & $14.37 \pm 0.03$ & $3.00 \pm 0.03$ & $2.83 \pm 0.08$ & $193.5 \pm 0.1$ & $4.83 \pm 0.05$ & 18.4 & 249 \\
\hline & 4,900 & 8 & & 8.659 & $13.62 \pm 0.04$ & $3.13 \pm 0.04$ & $2.67 \pm 0.09$ & $189.7 \pm 0.1$ & $5.33 \pm 0.07$ & 19.2 & 259 \\
\hline & 2,900 & 8 & & 8.76 98] & $14.50 \pm 0.05$ & $3.51 \pm 0.08$ & $1.85 \pm 0.07$ & $192.1 \pm 0.1$ & $5.6 \pm 0.2$ & 18.7 & 253 \\
\hline & 1,900 & 8 & & 8.02 & $13.79 \pm 0.06$ & $3.22 \pm 0.08$ & $2.0 \pm 0.1$ & $197.8 \pm 0.1$ & $5.4 \pm 0.2$ & 17.6 & 238 \\
\hline & 300 & 8 & & 14.398 & $13.85 \pm 0.07$ & $2.03 \pm 0.03$ & $1.7 \pm 0.1$ & $191.5 \pm 0.3$ & $3.46 \pm 0.05$ & 18.8 & 254 \\
\hline & 6,000 & 22.5 & & 7.47 & $11.81 \pm 0.01$ & $\begin{array}{c}2.11 \pm 0.08 \\
2.56[154]\end{array}$ & $\begin{array}{c}1.98 \pm 0.04 \\
1.9 \pm 0.1[155]\end{array}$ & $\begin{array}{c}198.6 \pm 1.3 \\
200[31] \\
194[99]\end{array}$ & $4.15 \pm 0.15$ & 17.5 & 236 \\
\hline \multirow[t]{3}{*}{ MoGe } & 2,000 & 64 & \multirow[t]{3}{*}{80 [106] } & 5.8 [156] & $5.90 \pm 0.03$ & $1.35 \pm 0.12$ & $1.86 \pm 0.14$ & $239 \pm 2$ & $5.3 \pm 0.5$ & 9.2 & 214 \\
\hline & 5,000 & 64 & & 2.9156 & $6.28 \pm 0.07$ & $1.4 \pm 0.3$ & $2.1 \pm 0.4$ & $291 \pm 7$ & $5.2 \pm 1.1$ & 7.1 & 164 \\
\hline & 7,000 & 64 & & 2.9156 & $6.30 \pm 0.09$ & $\begin{array}{c}1.2 \pm 0.1 \\
1.49-2.23|157|\end{array}$ & $\begin{array}{l}1.9 \pm 0.3 \\
1 . 8 \longdiv { 1 5 8 | }\end{array}$ & $\begin{array}{l}292 \pm 3 \\
4 0 0 \longdiv { 1 0 7 1 }\end{array}$ & $5.7 \pm 1.0$ & 7.1 & 165 \\
\hline $\begin{array}{l}\mathrm{Ba}_{.6 .} \mathrm{K}_{0.4} \mathrm{BiO}_{3} \\
\text { experiment }\end{array}$ & 25,000 & 150 & $70[130]$ & $\begin{array}{ll}3.04[128] \\
\end{array}$ & $27.2 \pm 0.2$ & $\begin{array}{c}4.20 \pm 0.05 \\
4.5[159] \\
4.3[161]\end{array}$ & $\begin{array}{l}2.6 \pm 0.2 \\
2.0\left[\begin{array}{l}1601 \\
1.8[1621\end{array}\right.\end{array}$ & $\begin{array}{c}273.3 \pm 0.4 \\
289[129] \\
270[163] \\
340[164]\end{array}$ & $3.58 \pm 0.05$ & 10.5 & 218 \\
\hline $\begin{array}{l}\mathrm{HoNi}_{2} \mathrm{~B}_{2} \mathrm{C} \\
\text { experiment }\end{array}$ & 20,000 & 300 & $\begin{array}{l}12.5[165] \\
\text { from } \xi_{0}\end{array}$ & 1.45 166] & $5.7 \pm 0.4$ & $\begin{array}{l}1.1 \pm 0.2 \\
0.95 \lcm{167]}\end{array}$ & $2.0 \pm 1.4$ & $297 \pm 5$ & $4.4 \pm 0.9$ & 5.7 & 33.0 \\
\hline $\begin{array}{l}\mathrm{YNi}_{2} \mathrm{~B}_{2} \mathrm{C} \\
\text { experiment }\end{array}$ & 20,000 & 600 & $\begin{array}{l}39[168] \\
\text { from } \xi_{0}\end{array}$ & 2.1 [166] & $15.1 \pm 0.7$ & 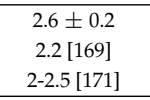 & $\begin{array}{c}2.4 \pm 1 \\
1.77 \llbracket 169]\end{array}$ & $\begin{array}{c}268 \pm 2 \\
350 \pm 50 \text { 170] }\end{array}$ & $3.9 \pm 0.3$ & 9.6 & 126 \\
\hline $\begin{array}{l}\mathrm{H}_{2} \mathrm{~S}(155 \mathrm{GPa}) \\
4 \text { param fit } \\
2 \text { param fit } \\
\text { experiment }\end{array}$ & 25,000 & 100 & $\begin{array}{l}88[20] \\
88[201 \\
\text { from } \xi_{0}\end{array}$ & $\begin{array}{c}9.8[20] \\
10.5[20]\end{array}$ & $204.6 \pm 0.1$ & $\begin{array}{c}26 \pm 3 \\
27.8 \pm 0.2\end{array}$ & $1.3 \pm 0.1$ & $\begin{array}{l}188 \pm 7 \\
189 \pm 2 \\
163[201\end{array}$ & $\begin{array}{c}2.95 \pm 0.3 \\
3.17 \pm 0.03\end{array}$ & $\begin{array}{l}23.2 \\
23.0\end{array}$ & $\begin{array}{l}580 \\
574\end{array}$ \\
\hline
\end{tabular}

Table 6.1: Fit parameters derived from fitting $J_{c}(s f, T)$ for $s$-wave superconductors (with selected examples shown in figure 6.1). $B_{c 1}$ and $B_{c}$ are calculated from $\lambda_{0}$. Reproduced from table 3 in the appendix of [132] without the $B_{x}$ and $B_{y}$ values. References next to the $J_{c}(0)$ values are where the $J_{c}(s f, T)$ data was obtained. 


\begin{tabular}{|c|c|c|c|c|c|c|c|c|c|c|}
\hline $\begin{array}{l}\text { Material/ } \\
\text { geometry }\end{array}$ & $\begin{array}{r}2 a \\
\mathrm{~nm}\end{array}$ & $\begin{array}{r}2 b \\
\mathrm{~nm}\end{array}$ & $\kappa$ & $\begin{array}{c}J_{c}(0) \\
\mathrm{MA} / \mathrm{cm}^{2} \\
\end{array}$ & $\begin{array}{l}T_{c} \\
(\mathrm{~K}) \\
\end{array}$ & $\begin{array}{c}\Delta_{0} \\
(\mathrm{meV})\end{array}$ & $\Delta C / C$ & $\begin{array}{c}\lambda_{0} \\
(\mathrm{~nm})\end{array}$ & $2 \Delta_{0} / k_{B} T_{c}$ & $\begin{array}{l}B_{c 1} \\
(\mathrm{mT})\end{array}$ \\
\hline \multicolumn{11}{|l|}{$d$-wave } \\
\hline $\begin{array}{l}\mathrm{HgBa}_{2} \mathrm{CaCu}_{2} \mathrm{O}_{8} \\
\text { experiment }\end{array}$ & 450,000 & 250 & $123[172]$ & 10.4 [173] & $120 \pm 1$ & $\begin{array}{c}16.9 \pm 0.3 \\
32[174]\end{array}$ & $0.96 \pm 0.09$ & $\begin{array}{c}188.3 \pm 0.6 \\
145,188[175]\end{array}$ & 3.27 & 24.8 \\
\hline $\begin{array}{l}\mathrm{Bi}_{2} \mathrm{Sr}_{2} \mathrm{CaCu}_{2} \mathrm{O}_{8}+\mathrm{Zn} \\
\text { experiment }\end{array}$ & 10,000 & 360 & $170 \lcm{176]}$ & 10.1 [177] & $82.7 \pm 2.4$ & $\begin{array}{c}14.0 \pm 0.6 \\
2 0 . 5 \longdiv { 1 7 8 1 } \\
23 \sqrt{1801}\end{array}$ & $\begin{array}{c}0.76 \pm 0.17 \\
1.5 \square 143]\end{array}$ & $\begin{array}{c}196 \pm 1 \\
180\lfloor 179]\end{array}$ & $3.93 \pm 0.17$ & 24.2 \\
\hline $\begin{array}{l}\mathrm{Bi}_{2} \mathrm{Sr}_{2} \mathrm{Ca}_{2} \mathrm{Cu}_{3} \mathrm{O}_{10} \\
\text { experiment }\end{array}$ & 20,000 & 100 & $170[181]$ & 14 [182] & $85.3 \pm 0.6$ & $\begin{array}{c}14.2 \pm 0.3 \\
30[180]\end{array}$ & $0.69 \pm 0.04$ & $\begin{array}{c}175.2 \pm 0.7 \\
151-155[183]\end{array}$ & $3.86 \pm 0.08$ & 30.2 \\
\hline $\begin{array}{l}\mathrm{Tl}_{2} \mathrm{Ba}_{2} \mathrm{CaCu}_{2} \mathrm{O}_{8} \\
\text { experiment }\end{array}$ & 12,000 & 650 & $150[184]$ & 12.9 & $103 \pm 1.9$ & $\begin{array}{l}19.1 \pm 0.8 \\
16-28[185]\end{array}$ & $\begin{array}{c}0.81 \pm 0.13 \\
0.6 \pm 0.1[186]\end{array}$ & $\begin{array}{c}179.4 \pm 0.8 \\
139,1 8 8 \longdiv { 1 8 7 1 }\end{array}$ & $4.30 \pm 0.18$ & 28.2 \\
\hline $\begin{array}{l}(\mathrm{Y}, \mathrm{Dy}) \mathrm{Ba}_{2} \mathrm{Cu}_{3} \mathrm{O}_{7} \\
\text { experiment }\end{array}$ & 500,000 & 850 & $95[31]$ & 31.8 [601 65] & $92.0 \pm 2.1$ & $\begin{array}{l}14.9 \pm 0.3 \\
16.7[188]\end{array}$ & $\begin{array}{c}0.78 \pm 0.14 \\
2.7[189]\end{array}$ & $\begin{array}{r}122.6 \pm 0.2 \\
125[115]\end{array}$ & $3.80 \pm 0.1$ & 55.4 \\
\hline $\begin{array}{l}(\mathrm{Y}, \mathrm{Dy}) \mathrm{Ba}_{2} \mathrm{Cu}_{3} \mathrm{O}_{7} \\
\text { experiment }\end{array}$ & 500,000 & 1400 & $95[31]$ & 26 [116] [117] & $87.5 \pm 0.4$ & $\begin{array}{l}12.8 \pm 0.1 \\
16.7 \overline{188]}\end{array}$ & $\begin{array}{c}1.23 \pm 0.06 \\
2.7[189]\end{array}$ & $\begin{array}{r}124.3 \pm 0.3 \\
125[115]\end{array}$ & $3.4 \pm 0.05$ & 53.9 \\
\hline $\begin{array}{l}\text { (Nd,Eu,Gd) } \mathrm{Ba}_{2} \mathrm{Cu}_{3} \mathrm{O}_{7} \\
\text { experiment }\end{array}$ & 50,000 & 50 & $95[31]$ & 30 [118] & $86.7 \pm 0.6$ & $\begin{array}{l}15.3 \pm 0.1 \\
16.7 \overline{188]}\end{array}$ & $\begin{array}{l}1.8 \pm 0.2 \\
2 . 7 \longdiv { 1 8 9 ] }\end{array}$ & $\begin{array}{c}130.5 \pm 0.2 \\
118[190]\end{array}$ & $4.1 \pm 0.04$ & 48.9 \\
\hline $\begin{array}{l}\mathrm{GdBa}_{2} \mathrm{Cu}_{3} \mathrm{O}_{7} \\
\text { experiment }\end{array}$ & 50,000 & 50 & $95[31]$ & 22.7 [118] & $85.4 \pm 0.8$ & $\begin{array}{l}14.9 \pm 0.2 \\
16.7[188]\end{array}$ & $\begin{array}{c}0.75 \pm 0.05 \\
2.7[189]\end{array}$ & $\begin{array}{c}143.1 \pm 0.2 \\
118[190]\end{array}$ & $4.05 \pm 0.07$ & 40.7 \\
\hline $\begin{array}{l}\mathrm{NdBa}_{2} \mathrm{Cu}_{3} \mathrm{O}_{7} \\
\text { experiment }\end{array}$ & 5,000 & 150 & $95 \lcm{311}$ & 28.9 [119] & $90.9 \pm 0.8$ & $\begin{array}{r}17.3 \pm 0.4 \\
16.7[188]\end{array}$ & $\begin{array}{c}1.56 \pm 0.16 \\
2.7[189]\end{array}$ & $\begin{array}{l}134 \pm 0.4 \\
118[190]\end{array}$ & $4.4 \pm 0.1$ & 46.4 \\
\hline
\end{tabular}

Table 6.2: Fit parameters derived from $J_{c}(s f, T)$ for $s$-wave superconductors (as shown in figure 6.3). $B_{c 1}$ and $B_{c}$ are calculated from $\lambda_{0}$. Reproduced from table 3 in the appendix of [132] without the $B_{x}$ and $B_{y}$ values. References next to the $J_{c}(0)$ values are where the $J_{c}(s f, T)$ data was obtained. 


\begin{tabular}{|c|c|c|c|c|c|c|c|c|c|c|c|}
\hline $\begin{array}{l}\text { Material/ } \\
\text { geometry }\end{array}$ & $\begin{array}{l}2 a \\
\mathrm{~nm}\end{array}$ & $\begin{array}{c}2 b \\
\mathrm{~nm}\end{array}$ & $\kappa$ & $\begin{array}{c}J_{c}(0) \\
\mathrm{MA} / \mathrm{cm}^{2}\end{array}$ & $\begin{array}{l}T_{c} \\
(\mathrm{~K})\end{array}$ & $\begin{array}{c}\begin{array}{c}\Delta_{0} \\
(\mathrm{meV})\end{array} \\
\text { (n) }\end{array}$ & $\Delta C / C$ & $\begin{array}{c}\lambda_{0} \\
(\mathrm{~nm})\end{array}$ & $2 \Delta_{0} / k_{B} T_{c}$ & $\begin{array}{l}B_{c 1} \\
(\mathrm{mT})\end{array}$ & $\begin{array}{c}B_{c} \\
(\mathrm{mT})\end{array}$ \\
\hline \multicolumn{12}{|l|}{ Nanowire } \\
\hline \multirow[t]{5}{*}{$\mathrm{Al}$} & 10 & 5 & \multirow{6}{*}{$0.03[31]$} & $9.23[73]$ & $1.48 \pm 0.01$ & $0.193 \pm 0.002$ & $1.3 \pm 0.1$ & $49.33 \pm 0.05$ & $3.03 \pm 0.05$ & - & 2.87 \\
\hline & 9.3 & 5 & & 8.68773 & $1.386 \pm 0.007$ & $0.195 \pm 0.002$ & $1.36 \pm 0.07$ & $50.4 \pm 0.09$ & $3.27 \pm 0.05$ & - & 2.75 \\
\hline & 8.4 & 5 & & $7.94 \quad 73]$ & $1.4 \pm 0.01$ & $0.205 \pm 0.002$ & $1.07 \pm 0.05$ & $51.89 \pm 0.04$ & $3.4 \pm 0.06$ & . & 2.60 \\
\hline & 7 & 5 & & $6.473]$ & $1.518 \pm 0.003$ & $0.182 \pm 0.001$ & $0.96 \pm 0.01$ & $55.77 \pm 0.01$ & $2.78 \pm 0.02$ & - & 2.25 \\
\hline & 5.4 & 5 & & 4.94 73] & $1.13 \pm 0.01$ & $0.14 \pm 0.001$ & $2.3 \pm 0.3$ & $60.79 \pm 0.06$ & $2.88 \pm 0.05$ & - & 1.89 \\
\hline experiment & & & & & & 0.179 [150] & $1.45191]$ & $\begin{array}{c}50 \pm 10 \text { [69] } \\
46-51[71] \\
51.5[72]\end{array}$ & & & \\
\hline \multirow[b]{2}{*}{. } & 100 & 65 & $3[192]$ & 0.0302 [147] & $0.82 \pm 0.03$ & $0.23 \pm 0.04$ & $4.3 \pm 1.7$ & $1114 \pm 5$ & $6.5 \pm 1.1$ & 0.21 & 0.56 \\
\hline & 100 & 65 & from $H_{c 0}$ & 0.0800 & $0.836 \pm 0.02$ & $\begin{array}{c}0.228 \pm 0.006 \\
0.115[151]\end{array}$ & $4.6 \pm 0.7$ & $\begin{array}{c}805.9 \pm 0.3 \\
30-60.5[1931\end{array}$ & $6.3 \pm 0.2$ & 0.41 & 1.08 \\
\hline $\begin{array}{l}\text { Amorph-W } \\
\text { experiment }\end{array}$ & 250 & 50 & $\begin{array}{c}117 \\
\text { from } \xi_{0}\left[\begin{array}{ll}194]\end{array}\right.\end{array}$ & 0.34 [194] & $4.7 \pm 0.2$ & $\begin{array}{c}0.9 \pm 0.2 \\
0.66\end{array}$ & $1.2 \pm 0.5$ & $738 \pm 13$ & $4.6 \pm 1.1$ & 1.59 & 50.0 \\
\hline $\begin{array}{l}\text { PbIn cylinder } \\
\text { experiment }\end{array}$ & 27.5 & - & $5[31]$ & 1.06 [145] & $7.6 \pm 0.6$ & $\begin{array}{c}1.6 \pm 0.1 \\
1.2-1.25[195]\end{array}$ & $\begin{array}{c}5 \pm 4 \\
3.53-3.71[135]\end{array}$ & $\begin{array}{l}295 \pm 2 \\
1 5 0 \longdiv { 3 1 ] }\end{array}$ & $4.8 \pm 0.4$ & 4.0 & 13.4 \\
\hline $\begin{array}{l}\text { MoN cylinder } \\
\text { experiment }\end{array}$ & 320 & - & $\begin{array}{c}54 \\
\text { from } \xi_{0}[101]\end{array}$ & $0.58[103]$ & $12.6 \pm 0.1$ & $\begin{array}{l}2.9 \pm 0.1 \\
1.94[196]\end{array}$ & $5 \pm 0.7$ & $\begin{array}{c}463 \pm 1 \\
440 \pm 40[102]\end{array}$ & $5.3 \pm 0.2$ & 3.45 & 58.7 \\
\hline $\mathrm{YBa}_{2} \mathrm{Cu}_{3} \mathrm{O}_{7}$ & 136 & 50 & $95[31]$ & 17.5 [197] & $81 \pm 1$ & $15.3 \pm 0.3$ & $1.4 \pm 0.2$ & $160.7 \pm 0.3$ & $4.38 \pm 0.01$ & 32.2 & 857 \\
\hline experiment & 50 & 48 & & 76.1198 & $81 \pm 1$ & $\begin{array}{l}16.9 \pm 0.4 \\
1 6 . 7 \longdiv { 1 8 8 }\end{array}$ & $\begin{array}{l}1.6 \pm 0.2 \\
2.7 \sqrt{189]}\end{array}$ & $\begin{array}{c}116.6 \pm 0.3 \\
125[115 !\end{array}$ & $4.8 \pm 0.1$ & 61.2 & 1626 \\
\hline
\end{tabular}

Table 6.3: Fit parameters derived from $J_{c}(s f, T)$ for $s$-wave superconductors (as shown in figure 6.4). $B_{c 1}$ and $B_{c}$ are calculated from $\lambda_{0}$. Reproduced from table 3 in the appendix of [132] without the $B_{x}$ and $B_{y}$ values. References next to the $J_{c}(0)$ values are where the $J_{c}(s f, T)$ data was obtained. 


\section{Chapter 7}

\section{Perpendicular Field Distribution measurements on superconducting tapes}

In chapter 4 we saw that the current distribution across a thin superconducting film is expected to be highly non-uniform across the width while in the Meissner state when the effective aspect ratio is larger than 1 . It was found however that at the self-field critical current, the thin films satisfy equation 3.1 over a large range of aspect ratios. This means that the local critical current must be uniformly distributed across the width. This predicted behaviour is tested in this chapter.

The current distribution in a conductor cannot be directly tested and so must be inferred by measuring the spatial variation of the magnetic field. Here measurements are shown which examine the profile of the magnetic field perpendicular to the tape, generated by a transport current in a rectangular superconductor. These measurements can be used to infer how the current distributes across the width of the superconductor.

This experiment is carried out on several commercial high temperature superconductor tapes with each producing similar results. The measurements confirm the inference in chapter 4 stating that, at the onset of dis- 
sipation, the current distribution across the width of a superconductor is uniform. The measured field data was first published in [199] and much of the chapter is outlined in [200] .

\subsection{Experimental setup}

A schematic diagram of the experimental setup is shown in figure 7.1. The perpendicular field above the sample is measured using an array of 7 Hall sensors (Arepoc THV-MOD). The active area of each Hall sensor is 0.01 $\mathrm{mm}^{2}$ with each positioned $1.5 \mathrm{~mm}$ from each other along a line. This means that the total width the sensor array spans is $9 \mathrm{~mm}$. The sensors are encased in plastic with a gap of $350 \mu \mathrm{m}$ between the line of the sensors and the bottom of the sensor casing.

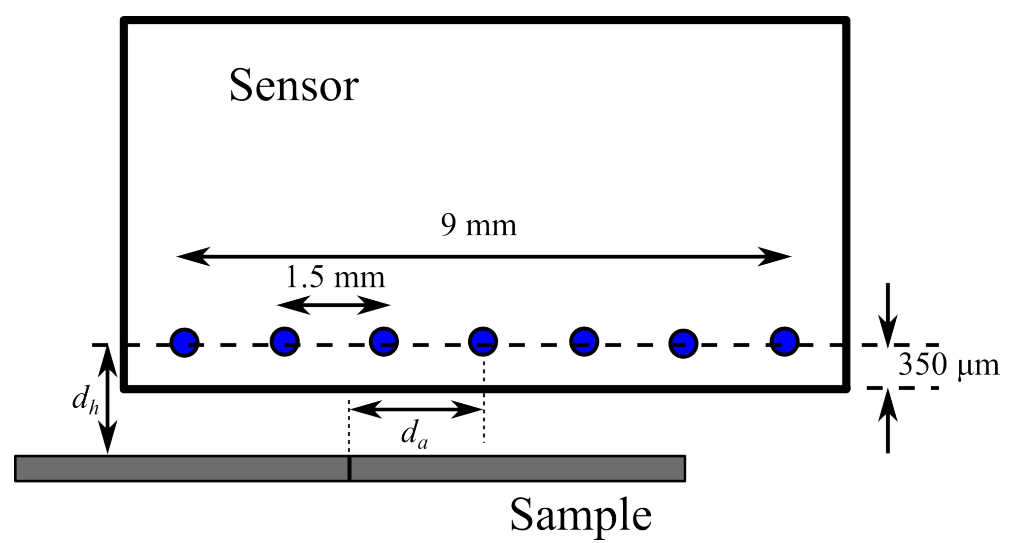

Figure 7.1: A diagram of the experimental setup used to measure the perpendicular component of the self-field generated by transporting electric current through a thin film superconductor tape. The Hall sensors used to measure the field are shown in blue and the thin film superconducting tape is shown in grey. The distances, $d_{h}$ and $d_{a}$, relate the position of the superconductor to the hall sensors (not to scale).

The sample that is to be studied is fastened to the bottom of the sensor casing. In figure 7.1 a distance, $d_{h}$, is shown as there is a small gap between 
Table 7.1: Manufacturers and dimensions of HTS commercial tapes used for measuring the field due to the transport current.

\begin{tabular}{|l|l|l|l|}
\hline Wire Manufacturer & Product Number & Width $(\mathrm{mm})$ & Thickness $(\mu \mathrm{m})$ \\
\hline Fujikura Ltd & FYSC-S10 10-0025-01 & 10 & 2.3 \\
\hline SuperPower Inc & SCS12050-AP M4-382-5 & 12 & 1.5 \\
\hline THEVA & TPL1100, ID 170468 & 12 & 2.6 \\
\hline
\end{tabular}

the bottom of the sensor casing and the superconductor tape. The alignment of the sample relative to the sensors, $d_{a}$, can be changed to measure the field directly above the sample or beyond the edge (in the figure it can be seen that some of the field measured is beyond the edge of the sample).

The sample is connected in a circuit to a power supply where the transport current applied to the sample can be controlled. For a typical measurement the current is increased in steps and the field is measured at every step. The whole sensor and sample was placed in a liquid nitrogen bath for cooling to ensure that the sample was in the superconducting state. The critical current was also measured in the usual way (see chapter 3).

\subsubsection{Superconducting tapes used}

Several commercial high temperature superconducting (HTS) tapes from three different wire manufacturers were used in this study. They were all $\mathrm{ReBa}_{2} \mathrm{Cu}_{3} \mathrm{O}_{y} 2 \mathrm{G}$ wires with the companies and their active superconductor dimensions shown in table 7.1 .

\subsection{Measurement data}

The left panel of figure 7.2 shows how the field strength changes as the current is increased. This is a SuperPower wire sample and it has been aligned such that the sensor array is close to its centre. The dotted line 
marks a change from non-linear behaviour to linear behaviour and the dashed line marks the measured critical current using an electric field criterion of $100 \mu \mathrm{V} / \mathrm{m}$. The field profile as a function of sensor position is shown in the right panels of figure 7.2 for a low current measurement of $10 \mathrm{~A}$ (top right) and for the critical current of $516 \mathrm{~A}$ (bottom right).
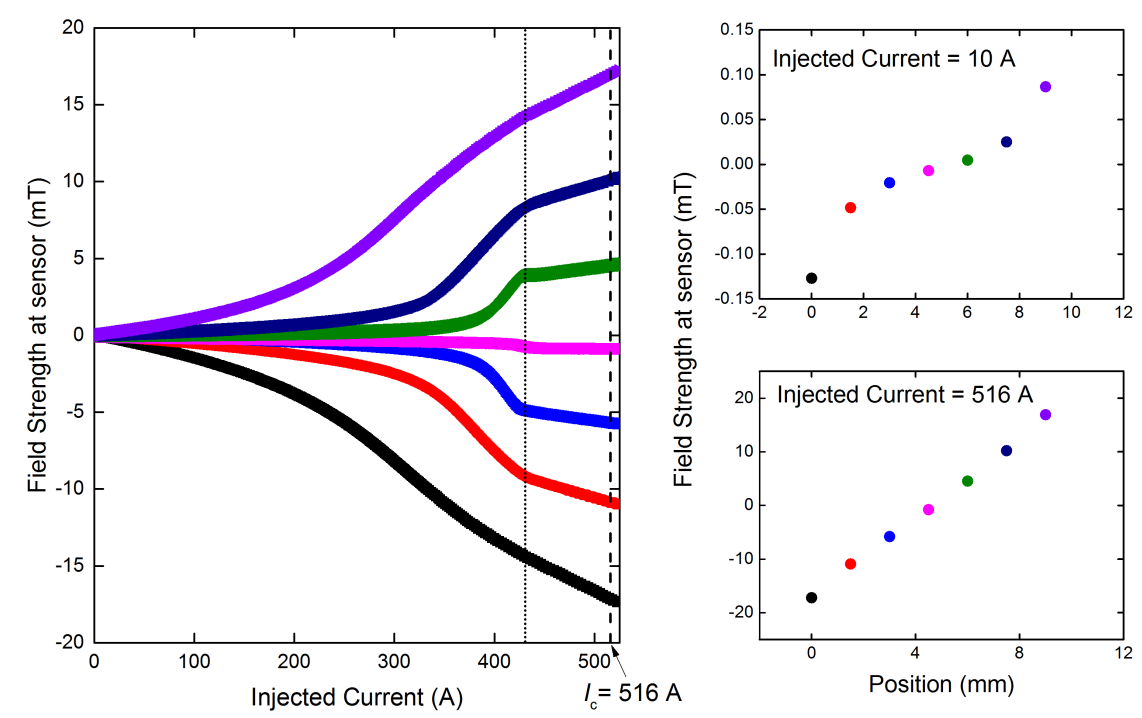

Figure 7.2: (Left) Perpendicular magnetic field strength at each sensor as a function of injected current in the sample. The sample is a $2 \mathrm{G}$ wire from SuperPower Inc and the sample has been positioned so its centre is aligned with the centre sensor. The data was first published in [199]. (Right top) Field strength vs position for an injected current of 10 A. (Right bottom) Field strength vs position for an injected current of $516 \mathrm{~A}$.

It can be seen quite clearly that the field profile changes in behaviour between the low and high current situations. The non-linear behaviour of the field vs current plot indicates that there is a redistribution of current as the injected current is increased. Also the linear behaviour at high currents indicates that the current is no longer redistributing and, as we will see, that the distribution of current in the sample has indeed become uniform. The other samples show similar results to that seen here. 
The angular dependence of the field can be seen in the data. The perpendicular field is maximal at the edge as expected, and goes to zero at the centre after which it flips to point in the other direction as one heads towards the other edge of the superconductor. This is expected for rectangular geometry.

The appearance of linear behaviour in the field before the measured critical current indicates that the critical state is reached at an earlier transport current value. This is to be expected as a voltage criterion was used in measuring the critical current (see chapter 3). This also gives a more fundamental way to determine what the critical current should be.

\subsection{Field distribution from current distribution}

For a given current distribution the resulting field can be calculated using the Biot-Savart law. For a rectangular sample of width, $2 a$, and thickness, $2 b$, the perpendicular magnetic field above the sample will follow the expression

$$
B_{\text {perp }}=\frac{\mu_{0}}{2 \pi} \int_{0}^{2 b} d y \int_{-a}^{a} J(x, y) \frac{\left(x-x_{0}\right)}{\left(x-x_{0}\right)^{2}+\left(y+y_{0}\right)^{2}} d x
$$

where $J(x, y)$ is the current density, and $x_{0}$ and $y_{0}$ are the coordinates of the sensor measured from the centre of the top edge of the sample.

At low currents the superconductor is expected to be in the LondonMeissner state where the current distribution across the width, averaged across the thickness will be that given by Rhoderick and Wilson [68] as

$$
J(x)=\frac{I}{\pi \sqrt{a^{2}-x^{2}}} .
$$

A uniform current distribution $(J(x)=$ constant $)$ can also be considered.

Plotted in figure 7.3 is the field profiles for both the Rhoderick and uniform current distributions with different values of $d_{h}$. When comparing them to the data in figure 7.2 it can be seen that at low current the data 
matches the field profile due to the Rhoderick current distribution (compare top right of figure 7.2) as there is an upturn and downturn at the edges. At the critical current the field profile matches the uniform current distribution (compare bottom right of figure 7.2). This behaviour was observed in the other superconducting tape samples as well which can be seen in a later plot.

The effect of changing $d_{h}$ sharpens or softens the peaks in the perpendicular field at the sample edges, depending on if you move closer or further away. If one was to change $d_{a}$ the whole distribution would just be translated along on the $x$ axis the amount $d_{a}$.

The linear behaviour of the field at high currents implies that a uniform current distribution will persist in this region. A uniform distribution of current is also expected for a normal conductor.

To plot the theoretical field profile against the measured data, the distance between the sensors and the sample is needed. Their positions relative to each other are characterized by two variables, $d_{h}$ and $d_{a}$ which are illustrated in figure 7.1. These were not explicitly measured in this experiment and so will need to be estimated in some way.

It has already been observed by comparing figures 7.2 and 7.3 the superconductor has a uniform current distribution at high currents which persist down to the current where the magnetic field behaviour is no longer linear. This gives us one way of finding the parameters $d_{a}$ and $d_{h}$, by fitting the field profile in this high current region as we know that it should have a uniform current distribution.

Also the superconductor will be in the Meissner state at low currents, and therefore should have a Rhoderick current distribution. This means that we could also fit the magnetic field profile at low currents (a limit of less than $0.1 I_{c}$ is used) to extract $d_{a}$ and $d_{h}$. An inspection of figure 7.3 shows that using the low-current data may be better for estimating $d_{h}$. If there are no sensors measuring the field profile in the region close to the edge of the sample the field profile due to a uniform current distribution 


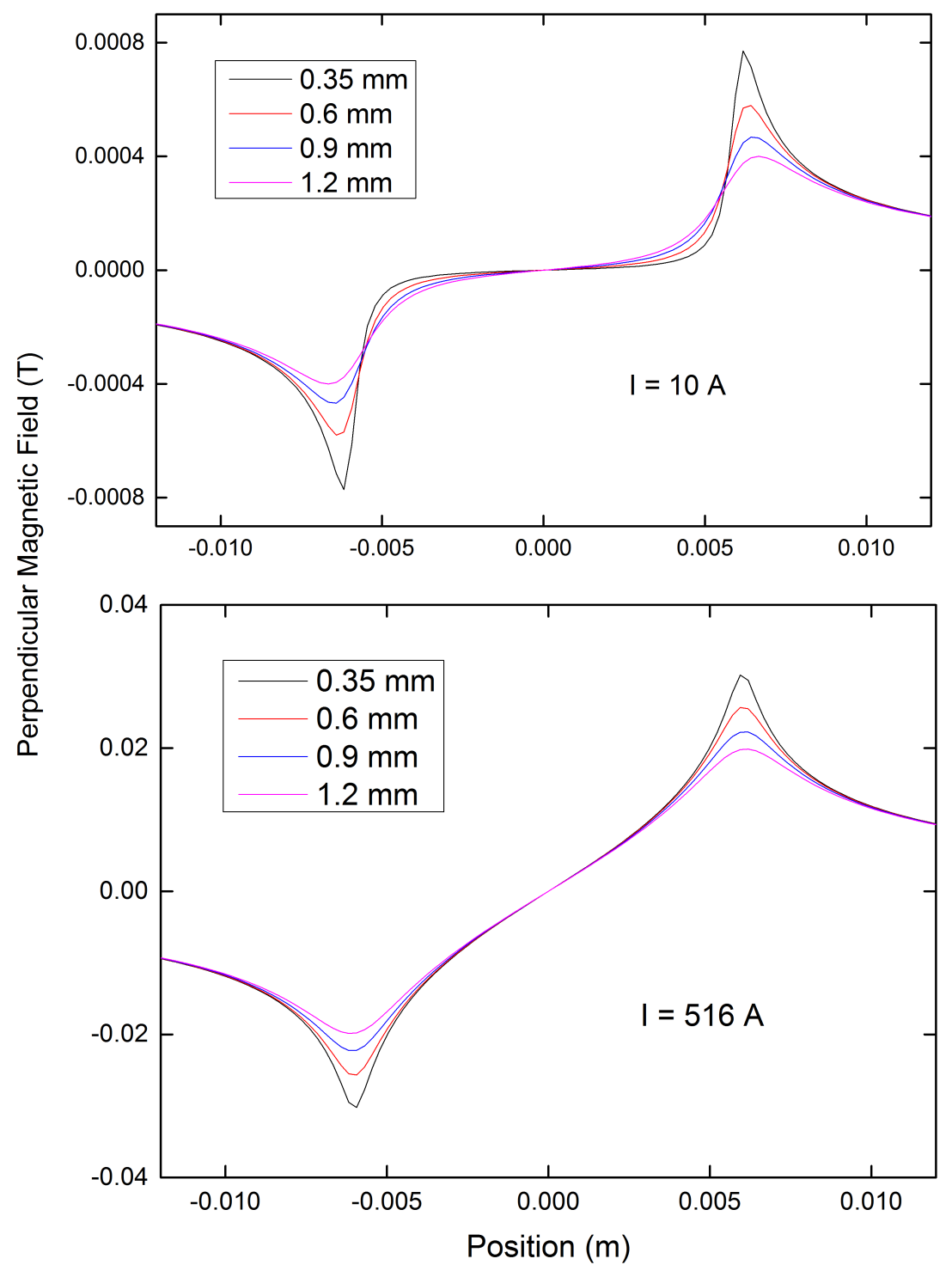

Figure 7.3: (Top) Perpendicular magnetic field distribution due to the Rhoderick current distribution where the injected current is $10 \mathrm{~A}$. (Bottom) Perpendicular magnetic field distribution due to the uniform current distribution where the injected current is $516 \mathrm{~A}$. The multiple lines are for different values of $d_{h}$. The width of the superconductor is $12 \mathrm{~mm}$, and the thickness is $1.5 \mu \mathrm{m}$. 
Table 7.2: The estimated values of $d_{a}$ and $d_{h}$ found by fitting the high current (uniform current distribution) and low current (Rhoderick current distribution) magnetic field distributions.

\begin{tabular}{|l|l|l|l|l|l|}
\hline \multirow{2}{*}{ Wire Manufacturer } & \multirow{2}{*}{ Position } & \multicolumn{2}{l|}{ Low Current Fit } & \multicolumn{2}{l|}{ High Current Fit } \\
\cline { 3 - 6 } & & $d_{a}(\mathrm{~mm})$ & $d_{h}(\mathrm{~mm})$ & $d_{a}(\mathrm{~mm})$ & $d_{h}(\mathrm{~mm})$ \\
\hline Fujikura Ltd & Centre & $4.71 \pm 0.01$ & $0.39 \pm 0.03$ & $4.8 \pm 0.1$ & $0.6 \pm 0.1$ \\
\hline SuperPower Inc & Centre & $4.74 \pm 0.06$ & $0.90 \pm 0.07$ & $4.59 \pm 0.09$ & $0.4 \pm 0.6$ \\
\hline SuperPower Inc & Centre & $3.29 \pm 0.03$ & $0.6 \pm 0.1$ & $3.6 \pm 0.1$ & $0.4 \pm 0.2$ \\
\hline SuperPower Inc & Off Centre & $1.48 \pm 0.06$ & $0.91 \pm 0.05$ & $1.6 \pm 0.2$ & $0.90 \pm 0.2$ \\
\hline SuperPower Inc & Off Centre & $1.60 \pm 0.08$ & $0.94 \pm 0.09$ & $1.7 \pm 0.2$ & $1 \pm 0.2$ \\
\hline THEVA & Off Centre & $0.89 \pm 0.09$ & $0.89 \pm 0.06$ & $1 \pm 0.3$ & $0.9 \pm 0.3$ \\
\hline
\end{tabular}

does not have much variation with $d_{h}$. In contrast the field profile due to the Rhoderick current distribution does have this variation and hence should give a better estimate of $d_{h}$.

But it should also be noted that fits of the high current region will better estimate $d_{a}$. This is because the field depends linearly on position with a good sized slope across the centre of the conductor unlike the low current field distribution which is close to flat making it harder to spot where the field changes direction.

Table 7.2 shows the result of fitting the magnetic field profile of both the high- and low-current regions to estimate $d_{a}$ and $d_{h}$. Equation 7.1 is used along with a uniform current distribution for the high-current region, and a Rhoderick current distribution for the low-current region.

It can be seen that the point made previously is confirmed. For samples that were off-centre the derived high-current $d_{h}$ position was roughly the same as that derived by using the low-current region, but for samples which were roughly centered, and the sample was wider than the span of the sensors, the derived high-current $d_{h}$ position did not match well with that derived from the low-current measurements. The $d_{a}$ position was matched within error for both cases. 
Figure 7.4 shows plots of the magnetic field profile data against the calculated field profile as described before for all the samples. The shape of the data clearly does follow that of the theory for both the low-current and high-current (in this case critical current) cases. This confirms the hypothesis of chapter 4 , that the distribution of current across the width of a rectangular superconductor, will be uniform in the critical state.

\subsection{Brandt and Indenbom fit to data}

There is still a need to address the current redistribution as the current is increased and the distribution goes from Rhoderick to uniform. One model to consider is that of Brandt and Indenbom [38].

Brandt and Indenbom considered a rectangular superconductor under self-field using the boundary condition that the current density cannot exceed some arbitrary critical current density, $j_{c}$ [38]. As current is injected into the superconductor it first reaches $j_{c}$ at the edges, and then must redistribute due to the condition that it may not exceed $j_{c}$ at any point.

This results in the formation of a region, $d$, at the edges saturating at the critical current. Outside of this region the current density falls away. The region expands as the total current increases causing the saturation of critical current to creep inwards until the whole width is at $j_{c}$, and this is the true critical current, $I_{c}$, of the sample. This is illustrated in figure 7.5 and is calculated using the equation

$$
\begin{aligned}
J(x) & =2 J_{c} \pi \arctan \left(\sqrt{\frac{a^{2}-d^{2}}{d^{2}-x^{2}}}\right),|x|<d \\
& =J_{c}, d<|x|<a
\end{aligned}
$$

where $d$ is the penetration of the flux into the superconductor and is given as $d=a\left(1-\left(I^{2} / I_{\max }^{2}\right)^{2}\right)$ and $I_{\max }=2 a J_{c}$.

The model is looking at the integrated current density, where the thickness has already been integrated across. They assumed that the mech- 

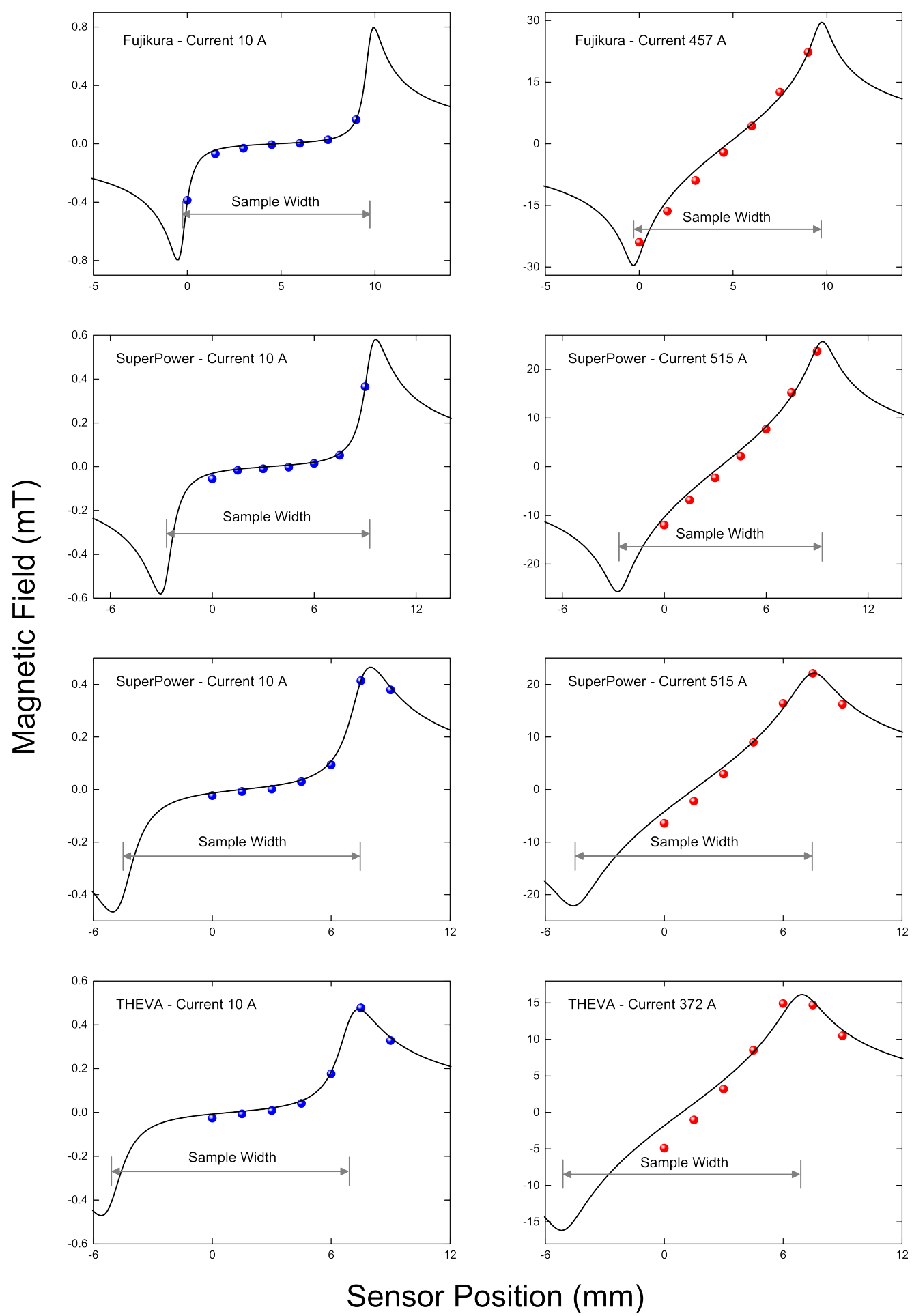

Figure 7.4: The field profiles both measured and calculated for all the samples. Two SuperPower Samples were excluded for the sake of space. The lines are calculated using equation 7.1 and a Rhoderick current distribution for low currents and a uniform current distribution for the critical currents. 


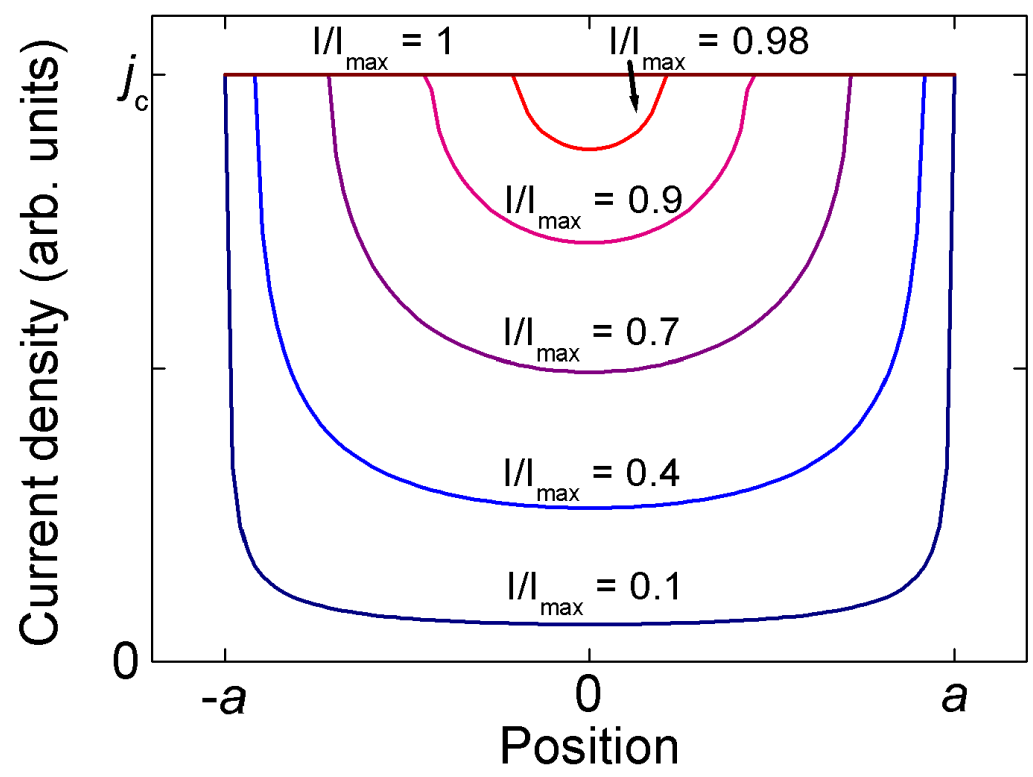

Figure 7.5: The Brandt and Indenbom current density distribution calculated for different injected currents [38]. It was calculated using equation 7.3

anism causing this critical current $j_{c}$ was due to the ingress of vortices, however the model is not derived with a specific mechanism in mind.

Figure 7.5 shows that in the Brandt and Indenbom model, the current distribution takes the form of the Rhoderick and Wilson distribution for low currents, and for critical currents the current distributes uniformly.

Figure 7.6 shows the magnetic field calculated using equation 7.3 and 7.1 against the data for the centered SuperPower sample. Here $I_{\max }$ has been set to the current at which the field changes from non-linear to linear behaviour.

It can be seen that the shape of the curve matches well and the absolute values are also a reasonable match, however the absolute value of the upper two lines do not match so well as the lower lines. The edge magnitude 


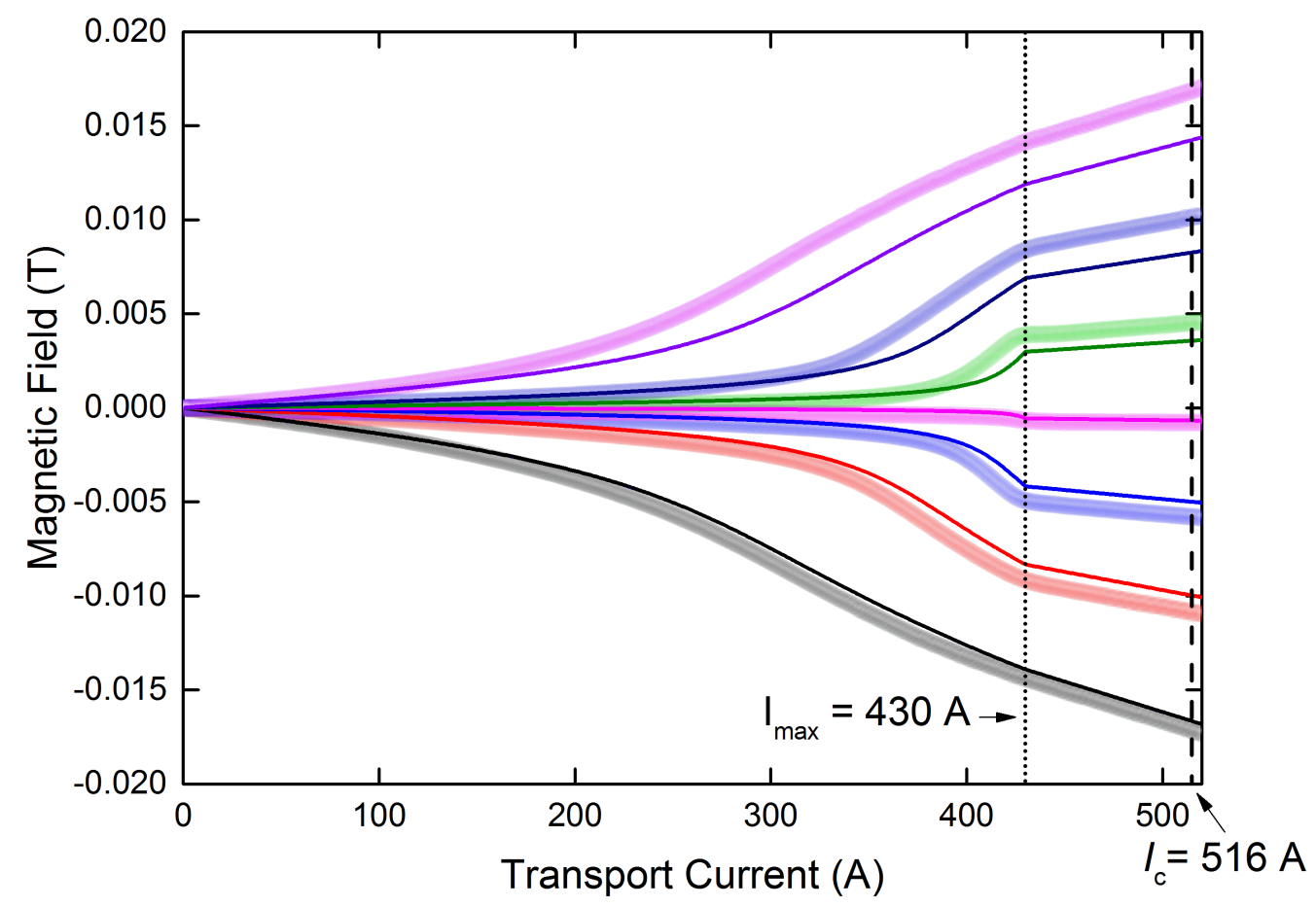

Figure 7.6: Measured data points (transparent points) and calculated values (lines) using the Brandt and Indenbom current distribution for the magnetic field profile of one of the SuperPower superconducting tapes.

of the magnetic field is around $12 \mathrm{mT}$ which is close to the value of $B_{c 1}(\| c)$ at $77 \mathrm{~K}$ [201] [202].

This can be interpreted as follows. The critical state starts at the edges of the film and penetrates further into the superconductor as the current increases until the critical current is reached. Here this critical state penetrates the whole width giving a uniform distribution.

As noted above Brandt and Indenbom presumed the mechanism limiting the current is the ingress of vortices, however their model is not based on any particular mechanism and so any critical state model will give the same results. In particular figure 4.2 specifically excludes a vortex model as the superconductor is still in the Meissner state. This new critical state has not been previously described and its origins remain unclear at this 
time.

In conclusion the measurements of the magnetic field profile for a rectangular superconductor showed that at low transport currents the current distribution follows that predicted by Rhoderick and Wilson [68]. When the critical state is reached the current distribution has become unifrom, confirming the hypothesis from chapter 4 . The redistribution of the current distribution also changes similarly to a model proposed by Brandt and Indenbom where the critical state starts at the edges of the film and penetrates furthur into the superconductor. 


\section{Chapter 8}

\section{Effects of impurity scattering on critical current}

In this chapter the self-field critical current is used as a probe to analyze impurity effects in the $\mathrm{YBCO}$ cuprate superconductor. When impurities are present in a $d$-wave superconductor, the superfluid density, $\rho$, is suppressed in a distinctive manner and in particular the superfluid density is suppressed much more rapidly than $T_{c}$ [203]. This should therefore show up distinctively in self-field critical current data and also will provide a good test of the ideas developed in previous chapters. Several YBCO+Zn thin film superconductors are used for this test and their preparation is described in chapter 3

\subsection{Impurity scattering in $d$-wave superconduc- tors: Theory}

The effects of impurities upon the superconducting state have been studied both theoretically and experimentally by a number of people [204] [203] [205]. Impurities work to weaken the superconducting state and this can be seen in the reduction of the superfluid density and of the critical 
temperature, $T_{c}$. Magnetic and non-magnetic impurities have different effects that depend on the symmetry of the order parameter and density of states [203] [206].

Early work by Loram [189] on YBCO doped with Zn impurities found evidence of strong pair breaking using specific heat measurements. Other work [207] was able to measure the superfluid density dependence on impurity concentration, and found that the relation closely follows that expected for a $d$-wave superconductor.

Here we examine the case of a $d$-wave superconductor with nonmagnetic impurities. In a $d$-wave superconductor impurity scattering causes a non-zero density of states near the Fermi energy which would otherwise be gapped. This causes a deviation from the expected linear $T$ dependence of the penetration depth, $\lambda(T)$, to a $T^{2}$ dependence at low temperatures [208] [209].

In the case of a $d$-wave superconductor with unitary scattering with a constant density of states around the Fermi surface Arberg and Carbotte [210] have given the strong-coupling expressions for the renormalized gap and renormalized Matsubara frequencies as

$$
\begin{aligned}
\tilde{\Delta}_{n \mathbf{k}} & =\eta_{\mathbf{k}} \pi T \sum_{m} \lambda(n-m)\left\langle\eta_{\mathbf{k}^{\prime}} \frac{\tilde{\Delta}_{m \mathbf{k}^{\prime}}}{\sqrt{\tilde{\omega}_{m}^{2}+\tilde{\Delta}_{m \mathbf{k}^{\prime}}^{2}}}\right\rangle \\
\tilde{\omega}_{n} & =\omega_{n}+\pi T \sum_{m} \lambda(n-m)\left\langle\frac{\tilde{\omega}_{m}}{\sqrt{\tilde{\omega}_{m}^{2}+\tilde{\Delta}_{m \mathbf{k}^{\prime}}^{2}}}\right\rangle+\pi \Gamma\left\langle\frac{\tilde{\omega}_{n}}{\sqrt{\tilde{\omega}_{n}^{2}+\tilde{\Delta}_{n \mathbf{k}^{\prime}}^{2}}}\right\rangle^{-1}
\end{aligned}
$$

where $\omega=(2 n-1) \pi T(n \in \mathbb{I})$ is the usual Matsubara frequency, $\eta_{\mathbf{k}}$ is the gap symmetry, $\Gamma$ is the impurity scattering constant linearly related to the density of impurities, $\langle\ldots\rangle$ is a normalized integration over the Fermi surface and

$$
\lambda(n-m)=2 N(0) \int \frac{\omega \alpha^{2} F(\omega)}{\omega^{2}+\left(\omega_{n}-\omega_{m}\right)^{2}} d \omega
$$

where $N(0)$ is the density of states at the Fermi energy and $\alpha^{2} F(\omega)$ is the electron boson spectral density. The superfluid density can then be com- 
puted using

$$
\rho_{i j}(T)=\frac{\pi T N(0)}{2 e^{2} \mu_{0}} \sum_{n}\left\langle v_{\mathbf{k}_{i}} v_{\mathbf{k}_{j}} \frac{\tilde{\Delta}_{n \mathbf{k}}^{2}}{\left(\tilde{\omega}_{n \mathbf{k}}^{2}+\tilde{\Delta}_{n \mathbf{k}}^{2}\right)^{3 / 2}}\right\rangle
$$

where $v_{F}$ is the Fermi velocity. To reduce to a BCS expression $\lambda(n-m)$ is assumed to be constant up to a cutoff frequency after which it will be zero as well as setting the $\lambda(n-m)$ term in the renormalized Matsubara frequency to be zero. This means that the renormalized gap will no longer depend on frequency.

As noted, based on our previous results we can use self-field critical currents as a tool to study the effects of impurities on the superfluid density in superconductors.

In particular the $\mathrm{YBa}_{2} \mathrm{Cu}_{3} \mathrm{O}_{y}$ superconductor will be studied here and $\mathrm{Zn}$ impurities will be added

\subsection{YBCO+Zn critical current data}

Several $\mathrm{YBCO}+\mathrm{Zn}$ thin film samples with various concentrations of $\mathrm{Zn}$ were made as specified in the methods section. For some of the samples, their temperature-dependent self-field critical current measurements were carried out on the device outlined in the methods section, and for the 3 samples which were measured down to $4.2 \mathrm{~K}$ the measurements were performed by Dr. Michael Eisterer and his group in Vienna.

Figure 8.1 shows the results for the samples measured at Robinson Research Institute (RRI). The graph looks similar to superfluid density measurements reported in [205] which would be expected as $J_{c}(s f, T) \propto \rho^{3 / 2}$. It can be seen that there is the expected drop in $T_{c}$ as the impurity concentration is increased. There is also a drop in the magnitude of the self-field critical current density, $J_{c}(s f, T)$, which is to be expected if it follows the relationship $J_{c}(s f, T) \propto \rho^{3 / 2}$. The spread of $J_{c}(s f, T)$ values for samples of the same impurity concentration reflects the presence of weak-links which 


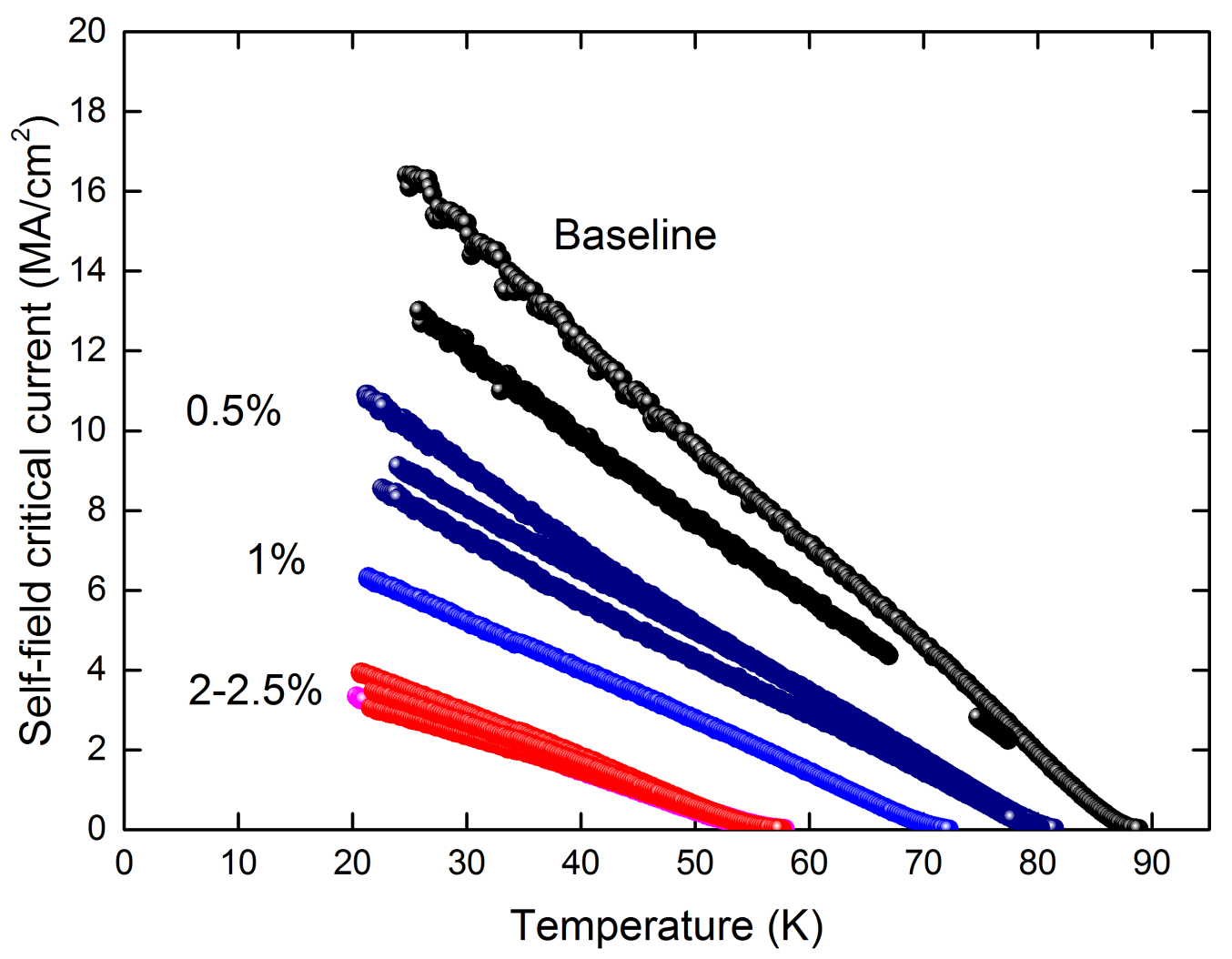

Figure 8.1: The self-field critical current densities of several different YBCO thin films with various concentrations of $\mathrm{Zn}$ impurities are shown plotted as a function of temperature here. Black data points are the "baseline" samples which have no added impurities present. The dark blue has a $0.5 \%$ concentration of $\mathrm{Zn}$ impurities added to the film, the blue has a $1 \%$ concentration of $\mathrm{Zn}$ impurities added to the film, the red has a $2-2.5 \%$ concentration of $\mathrm{Zn}$ impurities added to the film. The data here was measured in house at the Robin Research Institute. 
work to reduce the critical current. It can be seen however that $J_{c}(s f, T)$ (and by extension the superfluid density) reduces faster than $T_{c}$ which is the expected behaviour for impurity scattering.

It was found that after adding $\mathrm{Zn}$ past a concentration of $2-2.5 \%, T_{c}$ did not reduce anymore. This is probably a solubility limit, where the $\mathrm{Zn}$ atoms are no longer distributing throughout the $\mathrm{Cu} 0$ planes in a random fashion but instead would be forming concentrated regions. Tweaks of the sample synthesis method would be needed to get to higher concentrations of $\mathrm{Zn}$.

It is important to note that the concentrations specified refer to percentages of the total $\mathrm{Cu}$ content, as in $\mathrm{YBa}_{2}\left(\mathrm{Cu}_{1-x} \mathrm{Zn}_{x}\right)_{3} \mathrm{O}_{y}$. However $\mathrm{Zn}$ predominantly substitutes only for $\mathrm{Cu}$ on the $\mathrm{CuO}_{2}$ planes but not in the $\mathrm{CuO}$ chains. Because each formula unit has two of the former and only one of the latter the fraction on the $\mathrm{CuO}_{2}$ plane sites is $(3 / 2) x$. Thus the effective percentages in figure 8.1 are $0 \%, 0.75 \%, 1.5 \%$ and $3-3.75 \%$.

These data sets do not go to a low enough temperature to see the effect of impurity scattering on the temperature dependence of the superfluid density near $T=0$. In particular we desire to observe the predicted crossover from linear to quadratic behaviour. To explore the low temperature data several samples were sent to Vienna for testing.

Figure 8.2 shows the $J_{c}(s f, T)$ data measured at Vienna. The two samples with $\mathrm{Zn}$ impurities both show a leveling out of $J_{c}(s f, T)$ consistent with a transition from $\Delta \lambda(T) \propto T$ to $\Delta \lambda(T) \propto T^{2}(\Delta \lambda(T)=\lambda(T)-\lambda(0))$. Surprisingly there also is a leveling off in the self-field critical current of the "pure" sample where one was not expected. This might suggest intrinsic scattering is present even in pure samples.

Again the absolute magnitude of the self-field critical current is suppressed as before, however there seems to be little change in the temperature dependence from one impurity concentration to another. If all the curves are normalized they sit almost on top of each other especially the two $\mathrm{Zn}$ samples. This is consistent however with the weak coupling the- 

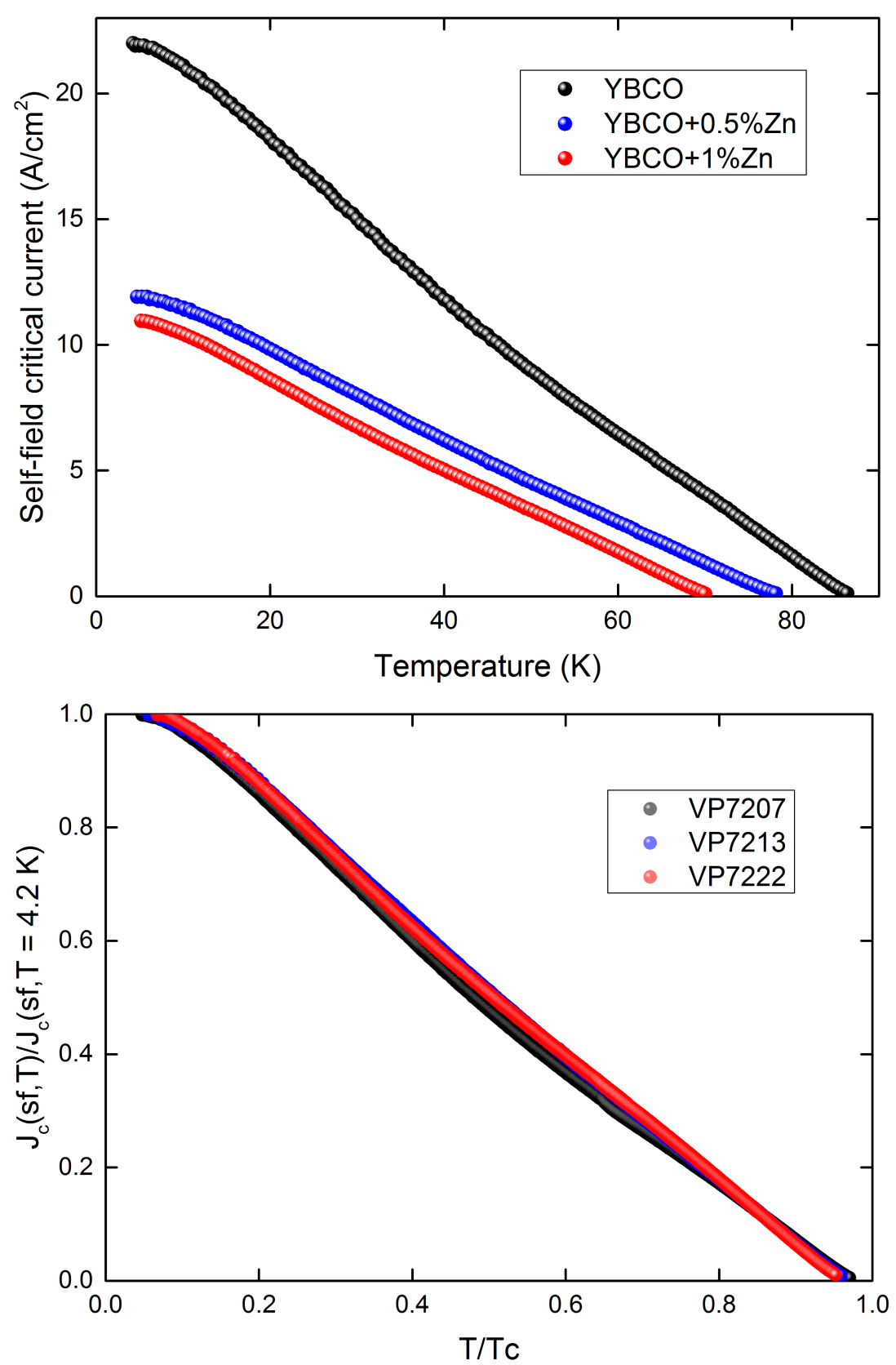

Figure 8.2: The self-field critical current densities of three different YBCO thin films with various concentrations of $\mathrm{Zn}$ impurities are shown in the top panel. The black is the "baseline" sample which has no impurities present. The blue has a $0.5 \%$ concentration of $\mathrm{Zn}$ impurities added to the film, and the red has a $1 \%$ concentration of $\mathrm{Zn}$ impurities added to the film. The data here was measured in Vienna by a group led by Dr. Michael Eisterer. The bottom panel shows the same data but normalized to compare the shape of the $T$-dependence. 
ory as can be seen in Sun and Maki [203]. In figure 6 in their paper they plot the superfluid density for different scattering constant values and it can be seen that the temperature dependence is quite similar apart from the pure case. This indicates that for superconductors of this kind the scattering constant might not be able to be extracted just from one temperature dependent measurement of $J_{c}(s f, T)$ or $\rho_{s}(T)$. The $T_{c}$ and $\lambda(0)$ may need to be known of both a superconductor with and without the added impurities.

Nonlocal electrodynamics can cause a transtion from a $T$ to $T^{2}$ dependence in the penetration depth [211]. This is expected to happen below a cross-over temperature $k_{B} T^{*}=(\xi(0) / \lambda(0)) \Delta(0)$. For YBCO this temperature is around $2 \mathrm{~K}$ and so the leveling out of $J_{c}(s f, T)$ in the pure samples, occuring as it does near $5 \mathrm{~K}$, is probably not due to nonlocal effects.

There is evidence however that surface bound Andreev states can cause an upturn in the penetration depth [212]. Experiments measuring the penetration depth in YBCO crystals cut along different crystallographic axes showed an upturn for several of those geometries. The variation from a linear $T$ dependence started at around 6-7 $\mathrm{K}$ in most cases and the penetration depth became flat as early as $4 \mathrm{~K}$ for the most extreme case turning upwards after. This effect was seen to be suppressed by a small magnetic field of about $10 \mathrm{mT}$ [212].

Using the equation for the surface field $B_{s}=\mu_{0} b J_{c}$ the field along the width at the surface will be around $100 \mathrm{mT}$. This will decay into the sample as $\sinh (y / \lambda)$ and using the current distribution from chapter 4 the field will drop to $10 \mathrm{mT}$ at around $0.3 b$.

According to the fields calculated here this Andreev effect is expected to be suppressed at the edges, however within the superconductor the field quickly drops away to zero, and the effect may still be present within the greater bulk of the sample. Field dependent studies of the low-temperature $J_{c}(s f, T)$ would help to identify the mechanism here. Because a magnetic field was shown to suppress the effect experiments applying small fields 
up to $10 \mathrm{mT}$ may show an increase in $J_{c}(s f, T)$ at very low temperatures.

YBCO with $\mathrm{Zn}$ is generally considered to exhibit unitary scattering and previous experiments measuring the ground state superfluid density compare their results to the theory of a weak-coupling d-wave superconductor with unitary scattering [207].

The same is done in figure 8.3 where the three samples presented earlier in figure 8.2 are plotted. The data points are normalized using the baseline $T_{c}$ and $J_{c}(s f, T)$ values from the pure sample. The normalized superfluid density is calculated by using the previously described theory where $J_{c} \propto \rho^{3 / 2}$. The lowest temperature points are used as an approximation to the ground state (they occur around $4.2 \mathrm{~K}$ ).

Also plotted is data from the literature for $\mathrm{YBCO}$ where $\mathrm{Ca}$ has been used to reach the overdoped state [207] as well as data from [205]. These samples have varying amounts of $\mathrm{Zn}$ which suppress the superfluid density and critical temperature. Moun spin relaxation was used to measure the superfluid density here.

It can be seen that the data compares reasonably well. The $1 \%$ sample sits on the theory line and the $0.5 \%$ sample sits above. It is possible that there are more weak links present in the $0.5 \%$ sample resulting in a lower $J_{c}(s f, T)$. However, as is apparent in figure 8.3, it is notable that directly measured values of the superfluid density[207] also tend to reveal a larger than expected reduction in superfluid density due to the fact that, even at low concentrations, $\mathrm{Zn}$ atoms will substitute at nearest neighbour positions [213]

This super-supression can also be seen in the data of figure 8.1 which is plotted on figure 8.3 where the values of $J_{c}(s f, T)$ at $25 \mathrm{~K}$ have now been used as an approximation for the ground-state values. This gives the purple data points and it can be seen they follow more closely the muon spin relaxation data. 


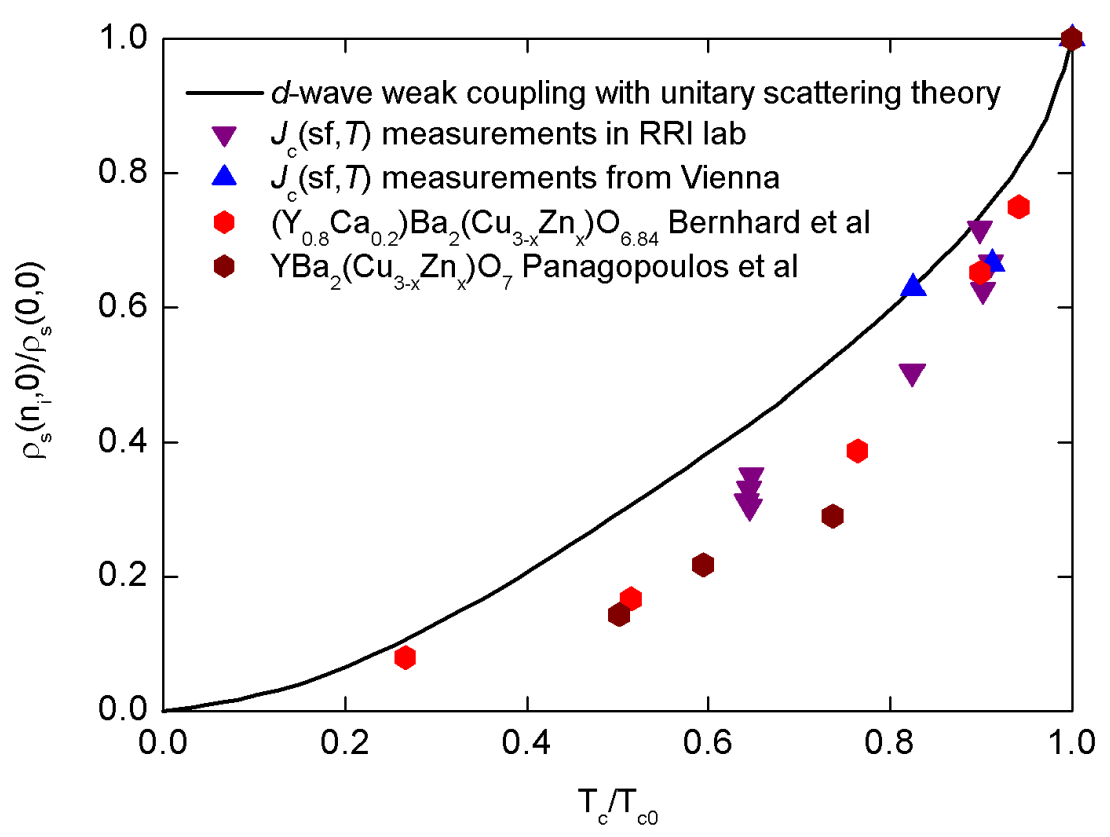

Figure 8.3: The normalized critical temperature is plotted vs the impurity dependent ground state superfluid density normalized by the impurity free ground state superfluid density. The line represents the theoretical curve for a d-wave superconductor with a cylindrical fermi surface in the unitary scattering limit. The red points are YBCO overdoped with calcium and added $\mathrm{Zn}$ impurities from [207] and the brown points are YBCO with added $\mathrm{Zn}$ impurities from [205]. The purple and blue triangles are from $J_{c}(s f, T)$ measurements of our $\mathrm{YBCO}+\mathrm{Zn}$ thin films undertaken at the (purple) Robinson Research Institute (RRI) and in (blue) Vienna by Dr. Michael Eisterer and his group. The normalized superfluid density was calculated using the relation between the critcal current density and the superfluid density: $J_{c} \rho^{3 / 2}$ 


\subsection{Conclusions}

Several thin-film samples of YBCO doped with added Zn impurities were synthesized and their self-field critical currents were measured. This selffield critical current was an analysed using the ideas from our previous chapters to extract the superfluid density. The superfluid density amplitude was suppressed by impurities in a way consistent with previous measurements from the literature using muon spin relaxation and it was suppressed in a way that is expected of a $d$-wave superconductor.

The temperature dependence in all cases followed a quadratic like saturation at low temperature. This was not expected for the pure sample and it is likely due to intrinsic scattering but possibly could be accounted for by Andreev surface bound states. The presence of Andreev bound states could be ruled out by studying the field dependence of the self-field critical current at very low temperatures and low fields. If the bound states are present a small increase of $J_{c}(s f, T)$ would be expected with increasing field. 


\section{Chapter 9}

\section{Deriving the geometric factors using the London equations}

The results in chapter 4 show that the total self-field critical current density can be divided into two multiplicative terms, $J_{c}(s f, T)=p f \cdot g f$. The pre-factor, $p f$, is the surface current density which is the universal condition giving the critical state and the geometric factor, $g f$, is given by the distribution of the current across the thickness (and more generally across the width).

At this critical state the superconductor is in the Meissner state and so the London equations can be used to determine the geometric factor. Some other theory describing the free energy would have to be used to calculate the pre-factor, which is not the concern here. In this chapter the London theory is used to derive the geometric factor, first for the case of the anisotropic superconductor with a large aspect ratio, and then for superconductors of arbitrary aspect ratios. 


\subsection{Anisotropic London superconductors}

It was previously stated without derivation that the self-field critical current of the anisotropic cuprate superconductors followed the relation

$$
J_{c}(s f)=\frac{B_{c 1}(c)}{\mu_{0} \lambda_{a b}} \frac{\lambda_{c}}{b} \tanh \left(\frac{b}{\lambda_{c}}\right) .
$$

If the origins of the prefactor were associated merely with nucleation of vortices on the wide flat surfaces then a more complex result might be anticipated, so it is important to see the justification for this result. Equation 9.1 can be derived by taking the anisotropic London equation from [42],

$$
\mathbf{B}+\lambda_{\text {mean }}^{2} \nabla \times(\hat{m}(\nabla \times \mathbf{B}))=0
$$

where $\hat{m}$ is a 3 by 3 normalized mass tensor with diagonal components $m_{i i}=M_{i} / \bar{M}$. Here $M_{i}$ are the effective masses in each principal direction and $\bar{M}=\left(M_{x} M_{y} M_{z}\right)^{1 / 3}$. The penetration depth in each direction is given by $\lambda_{i}=\lambda_{\text {mean }} m_{i}^{1 / 2}$ where $\lambda_{\text {mean }}$ is a mean penetration depth.

By taking the curl the equation can be rearranged in terms of current density to give

$$
\mathbf{J}+\lambda_{\text {mean }}^{2} \nabla \times(\nabla \times(\hat{m} \mathbf{J}))=0 .
$$

Consider an anisotropic thin film superconductor with width $2 a$ in the $\hat{x}$ direction and thickness $2 b$ in the $\hat{y}$ direction. Let the crystalline $c$-axis be along the $\hat{y}$ direction and the transport current flowing in the $\hat{z}$ direction. If we consider that the superconductor has a penetration depth $\lambda_{a b}$ along the $\hat{x}-\hat{z}$ plane and a penetration depth $\lambda_{c}$ along the $\hat{y}$ axis, the resulting partial differential equation for the current density will then be

$$
J(x, y)=\lambda_{a b}^{2} \frac{\partial^{2} J}{\partial x^{2}}+\lambda_{c}^{2} \frac{\partial^{2} J}{\partial y^{2}}
$$

which has the solution

$$
J(x, y)=C_{x} \cosh \left(x / \lambda_{a b}\right)+C_{y} \cosh \left(y / \lambda_{c}\right) .
$$


The Cosh functions appear because of the condition $J(x, y)=J(-x, y)=$ $J(x,-y)=J(-x,-y)$. The constants will need to be determined by some boundary conditions.

At the critical state the constant $C_{x}$ can be set to zero because, as was found previously, the surface current distribution across the width of a rectangular superconductor is uniform at the critical state. It can be seen now that the relevant length scale is just $\lambda_{c}$. The constant $C_{y}$ can be found by setting the surface current to $\frac{B_{c 1}(c)}{\mu_{0} \lambda_{a b}}$, as was shown before. This results in the solution

$$
J(x, y)=\frac{B_{c 1}(c)}{\mu_{0} \lambda_{a b}} \frac{\cosh \left(y / \lambda_{c}\right)}{\cosh \left(b / \lambda_{c}\right)} .
$$

and integrating to get the total current gives the previously stated equation 9.1.

\subsection{Rectangular superconductor with arbitrary as- pect ratios}

In our published paper [132] a geometric factor was introduced to better describe the critical current behaviour when the aspect ratio of a rectangular sample was near unity. It was given as

$$
g(a, b, \lambda)=\frac{\lambda}{a} \tanh \left(\frac{a}{\lambda}\right)+\frac{\lambda}{b} \tanh \left(\frac{b}{\lambda}\right)
$$

and the particular limits are shown in table 9.1 .

\begin{tabular}{|l|l|l|}
\cline { 2 - 3 } \multicolumn{1}{c|}{} & $b>>\lambda$ & $b<<\lambda$ \\
\hline$a>>\lambda, a>>b$ & $\frac{\lambda}{b}$ & 1 \\
\hline$a \simeq b$ & $\frac{\lambda}{a}+\frac{\lambda}{b} \simeq \frac{2 \lambda}{a}$ & 2 \\
\hline
\end{tabular}

Table 9.1: The limits of the geometric factor $\frac{\lambda}{a} \tanh \left(\frac{a}{\lambda}\right)+\frac{\lambda}{b} \tanh \left(\frac{b}{\lambda}\right)$ introduced in [132]. 
The expected limits appear for three of the cases however for the fourth (where $a \simeq b$ and $b<<\lambda$ ) the limit is 2. This is not expected as it implies there is an unphysical doubling of the critical current in this limit. Rather, the geometric factor for $a \simeq b$ and $b<<\lambda$ should instead follow something like the expression $\frac{2 \lambda}{a} \tanh \left(\frac{a}{2 \lambda}\right)$ similar to the cylindrical case, which must be a reasonable approximation for a square cross-section and certainly will not differ by a factor of 2 .

This can be seen by considering the case of a square superconductor where $a>>$. The current flows in the edges, and it can be seen that there is a contribution to the current from both the surface regions of the width and thickness, resulting in a factor of 2 showing up as $\frac{2 \lambda}{a}$. However for the case $a<<\lambda$ there is only one contribution filling the whole cross-section resulting in a factor of 1 .

An intuitive approximation for the geometric factor is that given by the expression

$$
g_{1}(a, b, \lambda)=\frac{\lambda(a+b)}{a b} \tanh \left(\frac{a b}{\lambda(a+b)}\right)
$$

where it can be seen to reduce to the expected expression $\frac{\lambda}{b} \tanh \left(\frac{b}{\lambda}\right)$ when $a>>b$, or to the expression $\frac{2 \lambda}{a} \tanh \left(\frac{a}{2 \lambda}\right)$ when $a \simeq b$. It turns out that London theory can be used to derive this geometric factor.

Again consider the London equations for a rectangular superconductor with current flowing in the $\hat{z}$ direction and usual half width, $a$, and half thickness, $b$. The result is the partial differential equation

$$
J(x, y)=\lambda^{2} \frac{\partial^{2} J}{\partial x^{2}}+\lambda^{2} \frac{\partial^{2} J}{\partial y^{2}}
$$

which when using the conditions $J(x, y)=J(-x, y)=J(x,-y)=J(-x,-y)$ has the solution

$$
J(x, y)=C_{1} \cosh \left(\frac{x}{\lambda}\right)+C_{2} \cosh \left(\frac{y}{\lambda}\right) .
$$

Considering the previous results, it is expected that the current at the edges reaches the critical value $J_{c, s}$, on both the $\hat{y}-\hat{z}$ plane and the $\hat{z}-\hat{x}$ 
plane, and so the constants can be set by using the boundary conditions $J(a, 0)=J(0, b)=J_{c, s}$. This results in the equation

$$
J(x, y)=J_{c, s}\left(\frac{\cosh \left(\frac{b}{\lambda}\right)-1}{\cosh \left(\frac{a}{\lambda}\right) \cosh \left(\frac{b}{\lambda}\right)-1} \cosh \left(\frac{x}{\lambda}\right)+\frac{\cosh \left(\frac{a}{\lambda}\right)-1}{\cosh \left(\frac{a}{\lambda}\right) \cosh \left(\frac{b}{\lambda}\right)-1} \cosh \left(\frac{y}{\lambda}\right)\right)
$$

for the current distribution. This can be integrated to get the total current density with a final resulting geometric factor of

$$
\begin{aligned}
g_{2}(a, b, \lambda)= & \frac{\lambda}{a b}\left(\frac{\cosh \left(\frac{b}{\lambda}\right)-1}{\cosh \left(\frac{a}{\lambda}\right) \cosh \left(\frac{b}{\lambda}\right)-1} b \sinh \left(\frac{a}{\lambda}\right)\right. \\
& \left.+\frac{\cosh \left(\frac{a}{\lambda}\right)-1}{\cosh \left(\frac{a}{\lambda}\right) \cosh \left(\frac{b}{\lambda}\right)-1} a \sinh \left(\frac{b}{\lambda}\right)\right) .
\end{aligned}
$$

It should be noted that if the width and height are equal, the expression reduces to $\frac{2 \lambda}{a} \tanh \left(\frac{a}{2 \lambda}\right)$, as found for cylindrical geometry.

The two geometric factors, equations 9.8 and 9.12, can be compared with each other by plotting against $b^{*}=\lambda(a+b) / a b$. This is shown in figure 9.1. In the left-hand plot the functions are seen to sit on top of each other, whilst the right-hand plot shows the percentage difference between the two when the values of $a, b$, and $\lambda$ are randomly selected values in the range $10^{-9}$ to $10^{-1}$. This percentage difference is only significant in the range $b^{*}=0.1-5$ where the difference reaches a maximum of around $1.5 \%$. This fully justifies the use of the simpler expression (9.8) for all practical applications.

We recall that in section 5.2 we used the compound parameter $b^{*}$ defined in this way to deduce $\lambda(T)$ in the case of rectangular Sn samples of aspect ratio close to unity. The analysis reproduced a magnitude and temperature dependence that almost exactly matched independent direct experimental measurements.

A further investigation can be made into what happens when anisotropy is involved in the calculation. In this case we have the anisotropic London equation as before with the resulting partial differential equation 9.4 with 

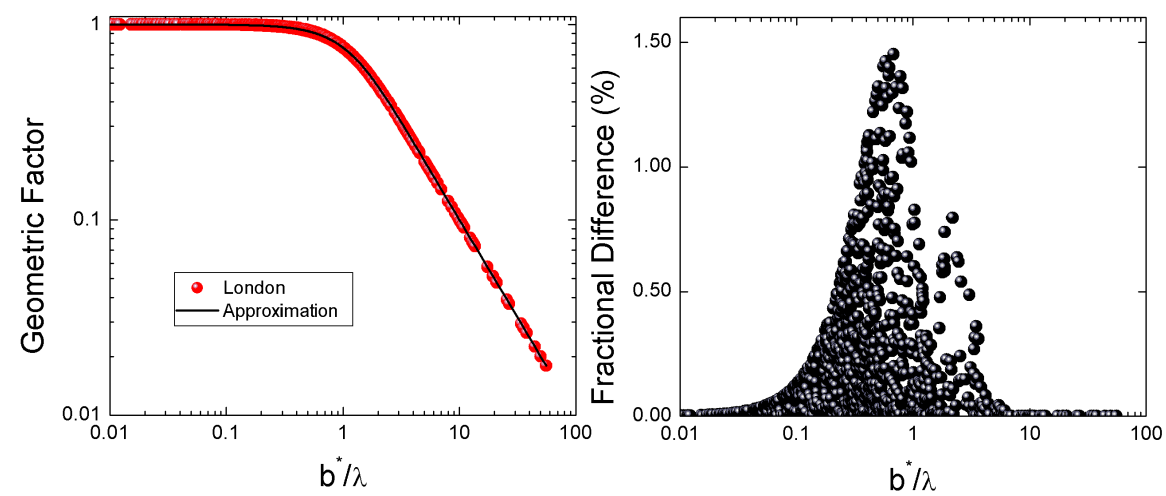

Figure 9.1: Comparison between the approximated geometric factor (equation 9.8) and that calculated using London theory (equation 9.12). The function values were calculated by taking random values of $a, b$, and $\lambda$ between $10^{-9}$ and $10^{-1}$. The left panel shows the London expression (red points) plotted on top of the approxmation (black line). The right panel shows the percentage difference between the two functions $\left(\left(g_{1}-g_{2}\right) / g_{1}\right)$.

a solution given by equation 9.5. In this case it would seem that the surface currents might be different due to the anisotropy of $\lambda$ and so the initial conditions will be taken to be $J(a, 0)=J_{s, a}, J(0, b)=J_{s, b}$. This results in the solution

$$
\begin{aligned}
J(x, y)= & \frac{J_{s, a} \cosh \left(\frac{b}{\lambda_{c}}\right)-J_{s, b}}{\cosh \left(\frac{a}{\lambda_{a b}}\right) \cosh \left(\frac{b}{\lambda_{c}}\right)-1} \cosh \left(\frac{x}{\lambda_{a b}}\right) \\
& +\frac{J_{s, b} \cosh \left(\frac{a}{\lambda_{a b}}\right)-J_{s, a}}{\cosh \left(\frac{a}{\lambda_{a b}}\right) \cosh \left(\frac{b}{\lambda_{c}}\right)-1} \cosh \left(\frac{y}{\lambda_{c}}\right)
\end{aligned}
$$


and it can be integrated to get the total current density as

$$
\begin{aligned}
J_{\text {total }}= & \frac{1}{4 a b} \int_{-a}^{a} \int_{-b}^{b} J(x, y) d x d y \\
= & \frac{1}{a b}\left[\left(J_{s, a} \cosh \left(\frac{b}{\lambda_{c}}\right)-J_{s, b}\right) b \lambda_{a b} \sinh \left(\frac{a}{\lambda_{a b}}\right)\right. \\
& \left.+\left(J_{s, b} \cosh \left(\frac{a}{\lambda_{a b}}\right)-J_{s, a}\right) a \lambda_{c} \sinh \left(\frac{b}{\lambda_{c}}\right)\right] /\left(\cosh \left(\frac{a}{\lambda_{a b}}\right) \cosh \left(\frac{b}{\lambda_{c}}\right)-1\right)
\end{aligned}
$$

The interesting, and as yet unresolved, question is whether in the Meissner critical state $J_{s, a}$ and $J_{s, b}$ differ or are equal. That will depend on the detailed origins of the critical surface current density. If they are equal then $J_{s}$ (which is equal to $J_{s, a}=J_{s, b}$ ) can be factored out and the equation, albeit complex, then defines the relevant anisotropic geometric form factor.

Here the geometric factors governing the total self-field critical current dependence on sample width and thickness have been derived. An interesting test of the current distributions derived here would be positiondependent magnetic field measurements of superconductors of varying aspect ratios in the self-field critical state. Such measurements would also confirm if the critical state surface current density for isotropic superconductors is the same across all surfaces, and for anisotropic superconductors differs across the different surfaces. 


\section{Chapter 10}

\section{Conclusions and prospects}

This chapter summarizes the work done in previous chapters. Some possible ideas are also put forward to explain the results.

\subsection{Summary of results}

The work presented here seeks to show that the self-field critical current in type II superconductors is a fundamental parameter of thermodynamic origins and therefore cannot be enhanced using engineering techniques. This knowledge also opens a new and straightforward way to estimate the relevant thermodynamic superconducting parameters using self-field critical current measurements.

The scaling behaviour revealed in chapter 4 shows that, at the critical state, the surface current density will reach the value $B_{c} / \mu_{0} \lambda$ for type I and $B_{c 1} / \mu_{0} \lambda$ for type II superconductors. This happens for both rectangular and cylindrical symmetries of many sizes as well as for different material types. It was also found for cases where the penetration depth was smaller than half the thickness of the superconductor, that the surface field was at or less than the critical field with a drop off for increasingly thinner samples. This fact implies that vortices are not involved in a determination of the self-field critical current. It also implies there is a fundamental current 
limit rather than a field limit.

The result here also implied that the current distribution across the width in rectangular geometries was uniform, something which was further explored in chapter 7 .

Chapter 6 explored the use of using measurements of the self-field critical current to determine the ground-state penetration depth, ground-state energy gap and specific heat jump at the critical temperature. These thermodynamic parameters were found by fitting the temperature-dependent critical current data to an extended BCS model.

In most cases the ground-state penetration depth was estimated with great accuracy. However because this estimation relied only on the magnitude of the self-field critical current, any presence of weak-links (which work to reduce the critical current) will cause the penetration depth to be over estimated. Therefore the most accurate analysis of the penetration depth requires weak-link free samples.

Estimates of the ground-state energy gap were also accurate in many cases. They tended to be better for the more conventional s-wave superconductors however many estimates for $d$-wave superconductors were still reasonable. And further, we note that many measurements of the superconducting gap in the cuprates are complicated by the presence of an often much larger pseudogap. For an accurate determination of the energy gap, only the temperature dependence of the critical current data is important (more specifically the low temperature data), and so the presence of weak-links is not so much of a factor.

The specific heat jump at the critical temperature was also estimated. Again the more conventional s-wave superconductors tended to fare better here. Estimating this parameter accurately was the least successful out of the three. The temperature dependent data near the critical temperature is the most important here to determine this. This data can often be the least accurate part of the whole temperature range due to the low critical currents being measured and often a statistical variation of the local 
critical temperature value due to defects and impurities.

In general however the temperature dependence of the critical current data matched well with that predicted by the model.

Recently we used this method to determine the thermodynamic parameters of the compressed $\mathrm{H}_{2} \mathrm{~S}$ system [140] where a determination of the energy gap by conventional methods would prove quite difficult.

This method can also be used to find multiple energy gaps in superconductors. This was carried out in a study of critical currents in the ultra-thin systems of Ga, FeSe, and 2H-Ta [214]. It is known that these materials often undergo an enhancement in their critical temperatures as they become thinner in dimension. By studying the self-field critical currents, it was shown that a second superconducting gap opens up as the critical temperature is enhanced. This seems to be correlated with the dimension of the film thickness falling below the coherence length.

Chapter7 7 reported measurements of the field perpendicular to the long surface of various thin-film superconducting tapes. The results confirmed the prediction that the current distribution along the width at the critical state would be uniform. In fact it crosses over from the highly nonuniform distribution expected in the Meissner state at low current to uniform at the critical current.

Chapter 8 explored the effects of non-magnetic impurities on the critical current in the $d$-wave superconducting cuprate system YBCO. A $d$ wave superconductor is expected to show a canonical suppression of the superfluid density as the density of impurities is increased. Since the selffield critical current can be used as a proxy to measure the superfluid density it should also show a similar suppression, but modified as in our case $J_{c}(s f) \propto \rho^{3 / 2}$. The measurements showed a suppression in the self-field critical current density consistent with conventional measurements of the superfluid density using techniques such as muon spin relaxation.

Overall the work here was able to establish that there is a fundamental limit to the self-field critical current and that it can be used as a probe of 
the superfluild density. There are some remaining challenges however.

As discussed in chapter 9 it would be interesting to test more thoroughly how anisotropy affects the self-field critical current density. The cuprate superconductor Bi-2223 with its very large anisotropy [215] could provide a rigorous test of equation 9.1. The difficulty perhaps is that samples with thickness well exceeding $\lambda_{c}$ would be needed. It must be noted that the doping dependence of $\lambda_{c}$ is quite extreme [215], but this may also provide another test. It may be possible with $1 \mathrm{G}$ wires where the overall thickness is around $200 \mu \mathrm{m}$.

The first challenge is to determine what is the origin behind this universal behaviour of the self-field critical current density. For type I superconductors it is clear that at the critical state a surface current density of $J_{s, \mathrm{I}}=B_{c} / \mu_{0} \lambda$ coincides with the depairing current density, however the surface current density of $J_{s, \mathrm{II}}=B_{c 1} / \mu_{0} \lambda$ for type II superconductors does not. It is generally far smaller. They do, however, exhibit the same scaling behaviour implying that $B_{c 1} / \mu_{0} \lambda$ may be some sort of type II depairing limit.

Because the measurement of the self-field critical current is straightforward, our approach here could be applied to many different problems, for example, critical current measurements could be carried out at the same time as other measurements to gain a better understanding of the superconductor under investigation. One particular example could be ionic liquid gating experiments. Ionic liquid gating has been used to change the carrier density (essentially doping) of various superconducting systems such as the transition metal dichalcogenides [216, 217, 218] and cuprates [219, 220]. Self-field critical current measurements could be used in conjunction with these experiments to better map out the phase diagrams of these materials including changes in the superfluid density.

Self-field critical current analysis could also be used to probe materials in which conventional experiments measuring properties like the gap or superfluid are more difficult. This was already seen in the case of $\mathrm{H}_{2} \mathrm{~S}$ 
under pressure [140], but could also be used in other high pressure experiments. The technique could also be used as a first probe of the superfluid density as it is a more accessible technique.

As discussed in chapter 8 it would be interesting to see if high quality thin films or crystals of YBCO showed linear behaviour of the self-field critical current as the temperature goes to zero. One might expect there to be a downturn due to the effect of Andreev bound states and this will have a characteristic field dependence.

To conclude we have presented a simple but powerful technique to probe the superconducting condensate. It shows, unexpectedly, that the self-field critical current is a fundamental parameter and this will be used in a wide range of experiments in the future to routinely characterize type II superconductors. Crucially this work establishes universal behaviour for all superconductors irrespective of their type, material or symmetry. 


\section{Bibliography}

[1] F. B. Silsbee, "A note on electrical conduction in metals at low temperatures," Journal of the Washington Academy of Sciences, vol. 6, no. 17, p. 597, 1916.

[2] M. Tinkham, Introduction to Superconductivity. United States of America: McGraw-Hill, Inc, second edition ed., 1996.

[3] G. E. Volovik, "Superconductivity with lines of gap nodes: density of states in the vortex," Journal of Experimental and Theoretical Physics, vol. 58, p. $469,1993$.

[4] X. Xu, "A review and prospects for $\mathrm{Nb}_{3} \mathrm{Sn}$ superconductor development," Superconductor Science and Technology, vol. 30, p. 093001, 2017.

[5] D. C. Larbalestier and P. J. Lee, "New developments in Niobium Titanium superconductors," IEEE Transactions Applied Superconductors, vol. 12, p. 1276, 1996.

[6] F. Lu, F. Kametani, and E. E. Hellstrom, "Optimization of a fluorine-free metal-organic deposition to fabricate $\mathrm{BaZrO}_{3}$-doped $\mathrm{YBa}_{2} \mathrm{Cu}_{3} \mathrm{O}_{7 \delta}$ film on RABiTS substrates," Superconductor Science and Technology, vol. 26, p. 045016, 2013.

[7] A. Xu, L. Delgado, N. Khatri, Y. Liu, V. Selvamanickam, D. Abraimov, J. Jaroszynski, F. Kametani, and D. C. Larbalestier, 
"Strongly enhanced vortex pinning from 4 to $77 \mathrm{~K}$ in magnetic fields up to $31 \mathrm{~T}$ in 15 mol.\% Zr-added (Gd, Y)-Ba-Cu-O superconducting tapes," Applied Physics Letters Materials, vol. 2, p. 046111, 2014.

[8] M. Leroux, K. J. Kihlstrom, S. Holleis, M. W. Rupich, S. Sathyamurthy, S. Fleshler, H. P. Sheng, D. J. Miller, S. Eley, L. Civale, A. Kayani, P. M. Niraula, U. Welp, , and W.-K. Kwok, "Rapid doubling of the critical current of $\mathrm{YBa}_{2} \mathrm{Cu}_{3} \mathrm{O}_{7 \delta}$ coated conductors for viable high-speed industrial processing," Applied Physics Letters Materials, vol. 107, p. 192601, 2015.

[9] F. Lu, F. Kametani, and E. E. Hellstrom, "Electric currents in REBaCuO superconducting tapes," Superconductor Science and Technology, vol. 30, p. 045010, 2017.

[10] J. Bardeen, L. N. Cooper, and J. R. Schrieffer, "Theory of superconductivity," Physical Review, vol. 108, no. 5, p. 1175, 1957.

[11] W. Meissner and R. Ochsenfeld, "Ein neuer effekt bei eintritt der supraleitfähigkeit," Naturwissenschaften, vol. 21, pp. 787-788, 1933.

[12] Introduction to Superconductivity. United States of America: McGrawHill, Inc, second edition ed., 1996.

[13] C. P. Poole, H. A. Farach, R. J. Creswick, and R. Prozorov, Superconductivity. London: Academic Press, second edition ed., 2007.

[14] H. K. Onnes, "Further experiments with liquid helium. D. on the change of electric resistance of pure metals at very low temperatures, etc. V. the disappearance of the resistance of mercury," Communications of the Physical Laboratory of Leiden University, vol. 122b, 1911.

[15] J. N. Rjabinin and L. W. Shubnikow, "Magnetic properties and critical currents of supra-conducting alloys," Nature, vol. 135, pp. 581$582,1935$. 
[16] F. Steglich, J. Aarts, C. D. Bredl, W. Lieke, D. Meschede, W. Franz, and H. Schäfer, "Superconductivity in the presence of strong pauli paramagnetism: $\mathrm{CeCu}_{2} \mathrm{Si}_{2}$," Physical Review Letters, vol. 43, p. 1892, 1979.

[17] D. Jérome, A. Mazaud, M. Ribault, and K. Bechgaard, "Superconductivity in a synthetic organic conductor (TMTSF)2PF 6," Journal de Physique Lettres, vol. 41, no. 4, pp. 95-98, 1980.

[18] J. G. Bednorz and K. A. Müller, "Possible high $t_{c}$ superconductivity in the Ba-La-Cu-O system," Zeitschrift für Physik B Condensed Matter, vol. 64, pp. 189-193, 1986.

[19] Y. Kamihara, H. Hiramatsu, M. Hirano, R. Kawamura, H. Yanagi, T. Kamiya, and H. Hosono, "Iron-based layered superconductor: LaOFeP," Journal of the American Chemical Society, vol. 128, no. 31, pp. 10012-10013, 2006.

[20] A. P. Drozdov, M. I. Eremets, I. A. Troyan, V. Ksenofontov, and S. I. Shylin, "Conventional superconductivity at 203 kelvin at high pressures in the sulfur hydride system," Nature, vol. 525, pp. 73-76, 2015.

[21] J. R. Schrieffer, "The microscopic theory of superconductivity," Journal of Superconductivity, vol. 4, no. 5, pp. 317-320, 1991.

[22] M. R. Schafroth, "Coulomb interaction and the Meissner-Ochsenfeld effect," Il Nuovo Cimento, vol. 9, no. 3, pp. 317-320, 1952.

[23] L. N. Cooper, "Bound electron pairs in a degenerate fermi gas," Physical Review, vol. 104, no. 4, p. 1189, 1956.

[24] M. Tinkham, Introduction to Superconductivity. United States of America: McGraw-Hill, Inc, second edition ed., 1996. 
[25] H. Fröhlich, "Theory of the superconducting state. I. The ground state at the absolute zero of temperature," Physical Review, vol. 79, no. 5 , p. $845,1950$.

[26] J. Bardeen and D. Pines, "Electron-phonon interaction in metals," Physical Review, vol. 99, no. 4, p. 1140, 1955.

[27] C. C. Tsuei and J. R. Kirtley, "d-Wave pairing symmetry in cuprate superconductors-fundamental implications and potential applications," Physica C: Superconductivity, vol. 367, pp. 1-8, 2000.

[28] H. Won and K. Maki, " $d$-wave superconductor as a model of high- $t_{c}$ superconductors," Physical Review B, vol. 49, no. 2, p. 1397, 2017.

[29] N. R. Lee-Hone, J. S. Dodge, and D. M. Broun, "Disorder and superfluid density in overdoped cuprate superconductors," Physical Review B, vol. 96, no. 2, p. 024501, 2017.

[30] J. B. Ketterson and S. N. Song, Superconductivity. Cambridge, United Kingdom: Cambridge University Press, 1999.

[31] C. P. Poole, H. A. Farach, R. J. Creswick, and R. Prozorov, Superconductivity. London: Academic Press, second edition ed., 2007.

[32] B. S. Chandrasekhar and D. Einzel, "The superconducting penetration depth from the semiclassical model," Annalen der Physik, vol. 505, p. 535546, 1993.

[33] H. K. Onnes, "Threshhold current in mercury," Communications of the Physical Laboratory of Leiden University, vol. 133a, p. 3, 1911.

[34] D. Dew-Hughes, "The critical current of superconductors: an historical review," Low Temperature Physics, vol. 27, no. 9-10, p. 713, 2001.

[35] A. M. Campbell and J. E. Evetts, "Flux vortices and transport currents in type II superconductors," Advances in Physics, vol. 50, pp. 1249-1449, 2001. 
[36] G. Blatter, M. V. Feigel'man, V. B. Geshkenbein, A. I. Larkin, and V. M. Vinokur, "Vortices in high-temperature superconductors," Reviews of Modern Physics, vol. 66, no. 4, p. 1125, 1994.

[37] L. London, "The electromagnetic equations of the supraconductor," Proceedings of the Royal Society A, vol. 149, pp. 71-88, 1935.

[38] E. H. Brandt and M. Indenbom, "Type-II-superconductor strip with current in a perpendicular magnetic field," Physical Review B, vol. 48, no. 17, pp. 12893-12906, 1993.

[39] V. L. Ginzburg and L. D. Landau, "On the theory of superconductivity," Zhurnal Éksperimental'noi i Teoreticheskoi Fiziki, vol. 20, pp. 1064$1082,1950$.

[40] L. P. Gor'kov, "Microscopic derivataion of the ginzburg-landau equations in the theory of superconductivity," Journal of Experimental and Theoretical Physics, vol. 9, no. 6, p. 1364, 1959.

[41] C. P. Poole, H. A. Farach, R. J. Creswick, and R. Prozorov, Superconductivity. London: Academic Press, second edition ed., 2007.

[42] J. B. Ketterson and S. N. Song, Superconductivity. Cambridge, United Kingdom: Cambridge University Press, 1999.

[43] A. A. Abrikosov, "The magnetic properties of superconducting alloys," Journal of Physics and Chemistry of Solids, vol. 2, pp. 199-208, 1957.

[44] K. K. Likharev, "Superconducting weak links," Reviews of Modern Physics, vol. 51, no. 1, p. 101, 1979.

[45] G. Wang, M. J. Raine, and D. P. Hampshire, "How resistive must grain boundaries in polycrystalline superconductors be, to limit $j_{c}$ ?," Superconductor Science and Technology, vol. 30, no. 10, p. 104001, 2017. 
[46] G. Wang, M. J. Raine, and D. P. Hampshire, "The cause of weaklink grain boundary behaviour in polycrystalline $\mathrm{Bi}_{2} \mathrm{Sr}_{2} \mathrm{CaCu}_{2} \mathrm{O}_{8}$ and $\mathrm{Bi}_{2} \mathrm{Sr}_{2} \mathrm{Ca}_{2} \mathrm{Cu}_{3} \mathrm{O}_{1} 0$ superconductors," Superconductor Science and Technology, vol. 31, no. 2, p. 024001, 2018.

[47] J.-Y. Lin, M. Gurvitch, S. K. Tolpygo, A. Bourdillon, S. Y. Hou, and J. M. Phillips, "Flux pinning in $\mathrm{YaBa}_{2} \mathrm{Cu}_{3} \mathrm{O}_{7 \delta}$ thin films with ordered arrays of columnar defects," Physical Review B, vol. 54, no. 18, p. R12717, 1996.

[48] A. Vostner, Y. Sun, H. Weber, Y. Cheng, A. Kuršumović, and J. Evetts, "Neutron irradiation studies on Y-123 thick films deposited by liquid phase epitaxy on single crystalline substrates," Physica C: Superconductivity, vol. 399, pp. 120-128, 2002.

[49] M. Eisterer, R. Fuger, M. Chudy, F. Hengstberger, and H. W. Weber, "Neutron irradiation of coated conductors," Superconductor Science and Technology, vol. 23, no. 1, p. 014009, 2009.

[50] M. Haruta, K. Saura, N. Fujita, Y. Ogura, A. Ichinose, T. Maeda, and $\mathrm{S}$. Horii, "Relationship between vortex pinning properties and $\mathrm{mi}$ crostructure in BaNbO-doped $\mathrm{YBa}_{2} \mathrm{Cu}_{3} \mathrm{O}_{y}$ and $\mathrm{ErBa}_{2} \mathrm{Cu}_{3} \mathrm{O}_{y}$ films," Physica C: Superconductivity, vol. 494, pp. 158-162, 2013.

[51] T. D. Withnell, K. R. Sch'oppl, J. H. Durrell, and H. W. Weber, "Effects of irradiation on vicinal YBCO thin films," IEEE Transactions on Applied Superconductivity, vol. 19, pp. 2925-2928, 2009.

[52] J. L. MacManus-Driscoll, S. R. Foltyn, Q. X. Jia, H. Wang, A. Serquis, L. Civale, B. Maiorov, M. E. Hawley, M. P. Maley, and D. E. Peterson, "Strongly enhanced current densities in superconducting coated conductors of $\mathrm{YBa}-2 \mathrm{Cu}_{3} \mathrm{O}_{7-x}+\mathrm{BaZrO}_{3}$," Nature Materials, vol. 3, pp. 439-443, 2004. 
[53] N. M. Strickland, E. F. Talantsev, J. A. Xia, N. J. Long, M. W. Rupich, $\mathrm{X}$. Li, and W. Zhang, "Flux pinning by barium stannate nanoparticles in MOD YBCO coated conductors," IEEE Transactions on Applied Superconductivity, vol. 19, no. 3, pp. 3140-3143, 2009.

[54] A. Rohatgi, "Webplotdigitizer." https://automeris.io/ WebPlotDigitizer, 2018.

[55] W.-H. Zhang, Y. Sun, J.-S. Zhang, F.-S. Li, M.-H. Guo, Y.-F. Zhao, H.-M. Zhang, J.-P. Peng, Y. Xing, H.-C. Wang, T. Fujita, A. Hirata, Z. Li, H. Ding, C.-J. Tang, M. Wang, Q.-Y. Wang, K. He, S.-H. Ji, X. Chen, J.-F. Wang, Z.-C. Xia, L. Li, Y.-Y. Wang, J. Wang, L.-L. Wang, M.-W. Chen, Q.-K. Xue, and X.-C. Ma, "Direct observation of hightemperature superconductivity in one-unit-cell FeSe films," Chinese Physics Letters, vol. 31, no. 1, p. 017401, 2014.

[56] B. P. P. Mallet, Ion-size effects on cuprate High Temperature Superconductors. PhD thesis, Victoria University of Wellington, 2013.

[57] A.-H. Puichaud, Electron Microscopy Imaging of Flux Pinning Defects in YBCO Superconductors. PhD thesis, Victoria University of Wellington, 2016.

[58] S. D. Obertelli, J. R. Cooper, and J. L. Tallon, "Systematics in the thermoelectric power of high- $t_{c}$ oxides," Physical Review B, vol. 46, no. 22, p. 14928(R), 1992.

[59] T. Matsushita, Flux Pinning in Superconductors. Berlin, Germany: Springer, 2007.

[60] N. M. Strickland, C. Hoffmann, and S. C. Wimbush, "A 1 kA-class cryogen-free critical current characterization system for superconducting coated conductors," Review of Scientific Instruments, vol. 85, p. 113907, 2014. 
[61] W. H. Warnes and D. C. Larbalestier, "Critical current distributions in superconducting composites," Cryogenics, vol. 26, pp. 643-653, 1986.

[62] T. F. Coleman and Y. Li, "On the convergence of reflective newton methods for large-scale nonlinear minimization subject to bounds," Mathematical Programming, vol. 67, no. 2, pp. 189-224, 1994.

[63] T. F. Coleman and Y. Li, "An interior trust region approach for nonlinear minimization subject to bounds," SIAM Journal on Optimization, vol. 6, pp. 418-445, 1996.

[64] E. F. Talantsev, W. P. Crump, and J. L. Tallon, “Universal scaling of the self-field critical current in superconductors: from subnanometre to millimetre size," Scientific Reports, vol. 7, no. 10010, 2017.

[65] E. F. Talantsev and J. L. Tallon, "Universal self-field critical current for thin-film superconductors," Nature Communications, vol. 6, no. $7820,2015$.

[66] C. P. Poole, H. A. Farach, R. J. Creswick, and R. Prozorov, Superconductivity. London: Academic Press, second edition ed., 2007.

[67] J. Pearl, "Current distribution in superconducting films carrying quantized fluxoids," Applied Physics Letters, vol. 5, no. 4, p. 65, 1964.

[68] E. H. Rhoderick and E. M. Wilson, "Current distribution in thin superconducting films.," Nature, vol. 194, pp. 1167-1168, 1962.

[69] R. Prozorov, R. W. Giannetta, A. Carrington, P. Fournier, R. L. Greene, P. Guptasarma, D. G. Hinks, and A. R. Banks, "Measurements of the absolute value of the penetration depth in high- $t_{c}$ superconductors using a low- $t_{c}$ superconductive coating," Applied Physics Letters, vol. 77, no. 25, p. 4202, 2000. 
[70] J. Romijn, T. M. Klapwijk, M. J. Renne, and J. E. Mooij, "Critical pairbreaking current in superconducting aluminum strips far below $t_{c}$," Physical Review B, vol. 26, p. 3648, 1982.

[71] R. E. Doezema, S. C. Whitmore, and J. N. Huffaker, "Quasiparticle magnetospectroscopy and penetration-depth anisotropy in superconducting aluminum," Physical Review B, vol. 34, no. 7, p. 4614, 1986.

[72] M. A. Biondi and M. P. Garfunkel, "Millimeter wave absorption in superconducting aluminum. II. calculation of the skin depth," Physical Review, vol. 116, no. 4, p. 862, 1959.

[73] P. Li, P. M. Wu, Y. Bomze, I. V. Borzenets, G. Finkelstein, and A. M. Chang, "Switching currents limited by single phase slips in onedimensional superconducting Al nanowires," Physical Review Letters, vol. 107, no. 13, p. 137004, 2011.

[74] L. G. Neumann and Y. H. Kao, “Temperature dependence of the critical current in one-dimensional indium strips," Journal of Low Temperature Physics, vol. 48, pp. 321-330, 1982.

[75] H. L. Watson and R. P. Huebener, "Current-induced resistive state in cylindrical type-I superconductors," Physical Review B, vol. 10, p. $4577,1974$.

[76] G. Peabody and R. Meservey, "Magnetic flux penetration into superconducting thin films," Physical Review B, vol. 6, no. 7, p. 2579, 1972.

[77] M. Tian, J. Wang, J. S. Kurtz, Y. Liu, M. H. W. Chan, T. S. Mayer, and T. E. Mallouk, "Dissipation in quasi-one-dimensional superconducting single-crystal Sn nanowires," Physical Review B, vol. 71, no. 10, p. 104521, 2005. 
[78] T. K. Hunt, "Critical-current behavior in narrow thin-film superconductors," Physical Review, vol. 151, no. 1, p. 325, 1966.

[79] D. H. Kim, K. E. Gray, J. D. Hettinger, J. H. Kang, and S. S. Choi, "Resistive measurement of the temperature dependence of the penetration depth of $\mathrm{Nb}$ in $\mathrm{Nb} / \mathrm{AlO}_{x} / \mathrm{Nb}$ Josephson junctions," Journal of Applied Physics, vol. 75, no. 12, p. 8163, 1994.

[80] B. W. Maxfield and W. L. McLean, "Superconducting penetration depth of niobium," Physical Review, vol. 139, p. A1515, 1965.

[81] G. P. Felcher, R. T. Kampwirth, K. E. Gray, and R. Felici, "Polarizedneutron reflections: A new technique used to measure the magnetic field penetration depth in superconducting niobium," Physical Review Letters, vol. 52, p. 1539, 1984.

[82] R. Huebener, R. Kampwirth, R. Martin, T. Barbee, and R. Zubeck, "Critical current density in superconducting niobium films," IEEE Transactions on Magnetics, vol. 11, pp. 344-346, 1975.

[83] A. Maisuradze, A. Yaouanc, R. Khasanov, A. Amato, C. Baines, D. Herlach, R. Henes, P. Keppler, and H. Keller, "Evidence for Cooper pair diffraction on the vortex lattice of superconducting niobium," Physical Review B, vol. 88, p. 140509(R), 2013.

[84] R. E. Schwall, G. R. Stewart, and T. H. Geballe, "Low-temperature specific heat of layered compounds," Journal of Low Temperature Physics, vol. 22, pp. 557-567, 1976.

[85] E. Navarro-Moratalla, J. O. Island, S. M. nas Valero, E. PinillaCienfuegos, A. Castellanos-Gomez, J. Quereda, G. Rubio-Bollinger, L. Chirolli, J. A. Silva-Guillén, N. Agraït, G. A. Steele, F. Guinea, H. S. J. van der Zant, and E. Coronado, "Enhanced superconductivity in atomically thin $\mathrm{TaS}_{2}$," Nature Communications, vol. 7, p. 11043, 2016. 
[86] S. Gygax, "Low-field magnetization measurements on small $\mathrm{TaS}_{2}$ (aniline) $)_{3 / 4}$ crystals," Journal of Low Temperature Physics, vol. 36, pp. 109-119, 1979.

[87] Y. Kashihara, A. Nishida, and H. Yoshioka, "Upper and lower critical fields of $\mathrm{TaS}_{2}$ (pyridine) $)_{1 / 2}$," Journal of the Physical Society of Japan, vol. 46, no. 4, pp. 1112-1118, 1979.

[88] P. Garoche, P. Manuel, J. J. Veyssié, and P. Molinié, “Dynamic measurements of the low-temperature specific heat of $2 \mathrm{H}-\mathrm{TaS}_{2}$ single crystals in magnetic fields," Journal of Low Temperature Physics, vol. 30, pp. 323-336, 1978.

[89] G. D. Cody, G. W. Cullen, and J. P. M. Jr, "Field and angular dependence of critical currents in $\mathrm{Nb}_{3} \mathrm{Sn}$ II," Reviews of Modern Physics, vol. 36, p. 95, 1964.

[90] M. Garber, S. Shen, J. Bussiere, and G. Morgan, "AC critical currents of commercial $\mathrm{Nb}_{3} \mathrm{Sn}$ tapes," IEEE Transactions on Magnetics, vol. 11, pp. 373-376, 1975.

[91] C. Buzea and T. Yamashita, "Review of the superconducting properties of $\mathrm{MgB}_{2}$," Superconductor Science and Technology, vol. 14, no. 11, p. R115, 2001.

[92] C. Panagopoulos, B. D. Rainford, T. Xiang, C. A. Scott, M. Kambara, and I. H. Inoue, "Penetration depth measurements in $\mathrm{MgB}_{2}$ : Evidence for unconventional superconductivity," Superconductor Science and Technology, vol. 64, p. 094514, 2001.

[93] C. Zhang, D. Wang, Z.-H. Liu, Y. Zhang, P. Ma, Q.-R. Feng, Y. Wang, and Z.-Z. Gan, "Fabrication of superconducting nanowires from ultrathin $\mathrm{MgB}_{2}$ films via focused ion beam milling," AIP Advances, vol. 5, no. 2, p. 027139, 2015. 
[94] C. Niedermayer, C. Bernhard, T. Holden, R. K. Kremer, and K. Ahn, "Muon spin relaxation study of the magnetic penetration depth in $\mathrm{MgB}_{2}$, " Superconductor Science and Technology, vol. 65, p. 094512, 2002.

[95] C. Zhang, Y. Wang, D. Wang, Y. Zhang, Q.-R. Feng, and Z.-Z. Gan, "Hybrid physicalchemical vapor deposition of ultrathin $\mathrm{MgB}_{2}$ films on $\mathrm{MgO}$ substrate with high $t_{c}$ and $j_{c}$ " IEEE Transactions on Applied Superconductivity, vol. 23, p. 7500204, 2013.

[96] C. G. Zhuang, S. Meng, C. Y. Zhang, Q. R. Feng, Z. Z. Gan, H. Yang, Y. Jia, H. H. Wen, and X. X. Xi, "Ultrahigh current-carrying capability in clean $\mathrm{MgB}_{2}$ films," Journal of Applied Physics, vol. 104, no. 1, p. 013924, 2008.

[97] S. Jin, H. Mavoori, C. Bower, and R. B. van Dover, "High critical currents in iron-clad superconducting $\mathrm{MgB}_{2}$ wires," Nature, vol. 411, p. $563565,2001$.

[98] A. Engel, H. Bartolf, A. Schilling, K. Il'in, M. Siegel, A. Semenov, and H.-W. Hübers, "Temperature- and field-dependence of critical currents in NbN microbridges," Journal of Physics: Conference Series, vol. 97, p. 012152, 2008.

[99] B. Komiyama, Z. Wang, and M. Tonouchi, "Penetration depth measurements of singlecrystal $\mathrm{NbN}$ films at millimeterwave region," Applied Physics Letters, vol. 68, p. 562, 1996.

[100] J. R. Clem, B. Bumble, S. I. Raider, W. J. Gallagher, and Y. C. Shih, "Ambegaokar-baratoffginzburg-landau crossover effects on the critical current density of granular superconductors," Physical Review B, vol. 35, no. 13, p. 6637, 1987. 
[101] D. Christen, S. Sekula, J. Ellis, J. Lewis, and J. Williams, “Formation, properties, and ion irradiation effects of hexagonal structure $\mathrm{MoN}$ thin films," IEEE Transactions on Magnetics, vol. 23, p. 1014, 1987.

[102] N. Haberkorn, Y. Y. Zhang, J. Kim, T. M. McCleskey, A. K. Burrell, R. F. Depaula, T. Tajima, Q. X. Jia, and L. Civale, “Upper critical magnetic field and vortex-free state in very thin epitaxial $\delta$-MoN films grown by polymer-assisted deposition," Superconductor Science and Technology, vol. 26, no. 10, p. 105023, 2013.

[103] J. Buh, V. Kabanov, V. Baranov, A. Mrzel, A. Kovič, and D. Mihailovic, "Control of switching between metastable superconducting states in $\delta$-MoN nanowires," Nature Communications, vol. 6, p. 10250, 2015.

[104] H. Lei, R. Hu, and C. Petrovic, "Critical fields, thermally activated transport, and critical current density of $\beta$-FeSe single crystals," Physical Review B, vol. 84, no. 1, p. 014520, 2011.

[105] B. L. T. Plourde, D. J. V. Harlingen, D. Y. Vodolazov, R. Besseling, M. B. S. Hesselberth, and P. H. Kes, "Influence of edge barriers on vortex dynamics in thin weak-pinning superconducting strips," Physical Review B, vol. 64, no. 1, p. 014503, 2001.

[106] B. L. T. Plourde, D. J. V. Harlingen, N. Saha, R. Besseling, M. B. S. Hesselberth, and P. H. Kes, "Vortex distributions near surface steps observed by scanning SQUID microscopy," Physical Review B, vol. 66, no. 5, p. 054529, 2002.

[107] M. L. Latimer, G. R. Berdiyorov, Z. L. Xiao, F. M. Peeters, and W. K. Kwok, "Realization of artificial ice systems for magnetic vortices in a superconducting MoGe thin film with patterned nanostructures," Physical Review Letters, vol. 111, no. 6, p. 067001, 2013. 
[108] R. T. Gordon, H. Kim, N. Salovich, R. W. Giannetta, R. M. Fernandes, V. G. Kogan, T. Prozorov, S. L. Bud'ko, P. C. Canfield, M. A. Tanatar, and R. Prozorov, "Doping evolution of the absolute value of the london penetration depth and superfluid density in single crystals of $\mathrm{Ba}\left(\mathrm{Fe}_{1-x} \mathrm{Co}_{x}\right)_{2} \mathrm{As}_{2}$," Physical Review B, vol. 82, no. 5, p. 054507, 2010.

[109] T. Tamegai, S. Pyon, Q. P. Ding, H. Inoue, H. Kobayashi, Y. Tsuchiya, Y. Sun, H. Kajitani, and N. Koizumi, "Comparison of $j_{c}$ characteristics in PIT wires based on $\mathrm{BaFe}_{2} \mathrm{As}_{2}$ with different substitutions," Journal of Physics: Conference Series, vol. 507, no. 2, p. 022041, 2014.

[110] L. Luan, T. M. Lippman, C. W. Hicks, J. A. Bert, O. M. Auslaender, J.-H. Chu, J. G. Analytis, I. R. Fisher, and K. A. Moler, "Local measurement of the superfluid density in the pnictide superconductor $\mathrm{Ba}\left(\mathrm{Fe}_{1-x} \mathrm{Co}_{x}\right)_{2} \mathrm{As}_{2}$ across the superconducting dome," Physical Review Letters, vol. 106, no. 6, p. 067001, 2011.

[111] T. J. Williams, A. A. Aczel, E. Baggio-Saitovitch, S. L. Bud'ko, P. C. Canfield, J. P. Carlo, T. Goko, H. Kageyama, A. Kitada, J. Munevar, N. Ni, S. R. Saha, K. Kirschenbaum, J. Paglione, D. R. SanchezCandela, Y. J. Uemura, and G. M. Luke, "Superfluid density and field-induced magnetism in $\mathrm{Ba}\left(\mathrm{Fe}_{1-x} \mathrm{Co}_{x}\right){ }_{2} \mathrm{As}_{2}$ and $\mathrm{Sr}\left(\mathrm{Fe}_{1-x} \mathrm{Co}_{x}\right.$ )$_{2} \mathrm{As}_{2}$ measured with muon spin relaxation," Physical Review B, vol. 82, no. 9, p. 094512, 2010.

[112] S. Mohan, T. Taen, H. Yagyuda, Y. Nakajima, T. Tamegai, T. Katase, $\mathrm{H}$. Hiramatsu, and $\mathrm{H}$. Hosono, "Transport and magnetic properties of Co-doped $\mathrm{BaFe}_{2} \mathrm{As}_{2}$ epitaxial thin films grown on $\mathrm{MgO}$ substrate," Superconductor Science and Technology, vol. 23, no. 10, p. 105016, 2010.

[113] H. J. Niu and D. P. Hampshire, "Critical parameters of disordered nanocrystalline superconducting Chevrel-phase $\mathrm{PbMo}_{6} \mathrm{~S}_{8}$, " Physical Review B, vol. 69, no. 17, p. 174503, 2004. 
[114] M. Decroux, O. .Fischer, and R.Chevrel, "Superconducting wires of $\mathrm{PbMo}_{6} \mathrm{~S}_{8}$, " Cryogenics, vol. 17, pp. 291-294, 1977.

[115] J. E. Sonier, S. A. Sabok-Sayr, F. D. Callaghan, C. V. Kaiser, V. Pacradouni, J. H. Brewer, S. L. Stubbs, W. N. Hardy, D. A. Bonn, R. Liang, and W. A. Atkinson, "Hole-doping dependence of the magnetic penetration depth and vortex core size in $\mathrm{YBa}_{2} \mathrm{Cu}_{3} \mathrm{O}_{y}$ : Evidence for stripe correlations near $1 / 8$ hole doping," Physical Review B, vol. 76, p. 134518, 2007.

[116] S. Fleshler, K. DeMoranville, J. G. Jr, X. Li, E. Podtburg, M. W. Rupich, S. Sathyamurthy, C. L. H. Thieme, D. Tucker, and L. Whitman, "Development status of amsc amperium wire," Journal of Physics: Conference Series, vol. 507, p. 022005, 2014.

[117] S. C. Wimbush and N. M. A. Strickland, "A high-temperature superconducting (HTS) wire critical current database," 2016.

[118] C. Cai, B. Holzapfel, J. Hänisch, L. Fernández, and L. Schultz, “High critical current density and its field dependence in mixed rare earth $(\mathrm{Nd}, \mathrm{Eu}, \mathrm{Gd}) \mathrm{Ba}_{2} \mathrm{Cu}_{3} \mathrm{O}_{7-\delta}$ thin films," Applied Physics Letters, vol. 84, no. 3, p. 377, 2004.

[119] Y. Li and K. Tanabe, "Influence of deposition parameters on superconductivity and surface morphology of $\mathrm{NdBa}_{2} \mathrm{Cu}_{3} \mathrm{O}_{7-\delta}$ films grown by pulsed laser deposition with a single crystal target," Physica C: Superconductivity, vol. 324, pp. 198-210, 1999.

[120] J. R. Thompson, Y. R. Sun, L. Civale, A. P. Malozemoff, M. W. McElfresh, A. D. Marwick, and F. Holtzberg, "Effect of flux creep on the temperature dependence of the current density in Y-Ba-Cu-O crystals," Physical Review B, vol. 47, p. 14440, 1993. 
[121] A. L. Schawlow and G. E. Devlin, "Effect of the energy gap on the penetration depth of superconductors," Physical Review B, vol. 113, no. 1, p. 120, 1959.

[122] E. Laurmann and D. Shoenberg, "Penetration of magnetic field into superconductors. II. measurements by the casimir method," Proceedings of the Royal Society A, vol. 198, p. 560, 1949.

[123] T. E. Faber and A. B. Pippard, "The penetration depth and highfrequency resistance of superconducting aluminium," Proceedings of the Royal Society A, vol. 231, p. 336, 1955.

[124] J. M. Lock and S. L. Bragg, "Penetration of magnetic fields into superconductors III. measurements on thin films of tin, lead and indium," Proceedings of the Royal Society A, vol. 208, p. 391, 1951.

[125] B. Mühlschlegel, "Die thermodynamischen funktionen des supraleiters," Zeitschrift für Physík, vol. 155, pp. 313-327, 1959.

[126] Y. d. Song and G. I. Rochlin, "Transition from bulklike behavior to josephson-junction-like behavior in superconducting microbridges," Physical Review Letters, vol. 26, no. 7, p. 416, 1972.

[127] Y. d. Song and G. I. Rochlin, Transition from bulk behavior to Josephsonjunction like behavior in superconducting microbridges. PhD thesis, U.C. Berkeley, 1971.

[128] M. T. D. v. H. R.A. Schweinfurth, C.E. Platt, "Electrical and magnetic transport properties of laserdeposited $\mathrm{Ba}_{1-x} \mathrm{~K}_{x} \mathrm{BiO}_{3}$ thin films," $A p$ plied Physics Letters, vol. 61, p. 480, 1992.

[129] W. D. Mosley, J. Z. Liu, A. Matsushita, Y. P. Lee, P. Klavins, and R. N. Shelton, "Preparation and superconductivity of $\mathrm{Ba}_{1-x} \mathrm{~K}_{x} \mathrm{BiO}_{3}$ single crystals," Journal of Crystal Growth, vol. 128, pp. 804-807, 1993. 
[130] S. N. Barilo, S. V. Shiryaev, V. I. Gatalskaya, J. W. Lynn, M. Baran, H. Szymczak, R. Szymczak, and D. Dew-Hughes, "Scaling of magnetization and some basic parameters of $\mathrm{Ba}_{1-x} \mathrm{~K}_{x} \mathrm{BiO}_{3+y}$ superconductors near $t_{c \prime}$ " Physical Review B, vol. 58, no. 18, p. 12355, 1998.

[131] W. N. Hardy, D. A. Bonn, D. C. Morgan, R. Liang, and K. Zhang, "Precision measurements of the temperature dependence of $\lambda$ in $\mathrm{YBa}_{2} \mathrm{Cu}_{3} \mathrm{O}_{6} .95$ : strong evidence for nodes in the gap function.," Physical Review Letters, vol. 70, p. 3999, 1993.

[132] E. F. Talantsev, W. P. Crump, and J. L. Tallon, “Thermodynamic parameters of single or multiband superconductors derived from selffield critical currents," Annalen der Physik (Berlin), vol. 529, no. 12, 2017.

[133] J. P. Carbotte and F. Marsiglio, "Electron-phonon superconductivity," in Superconductivity: Conventional and Unconventional Superconductors (K. H. Bennemann and J. B. Ketterson, eds.), vol. 1, ch. 3, pp. 74-153, Germany: Spinger, 2008.

[134] F. Gross, B. S. Chandrasekhar, D. Einzel, K. Andres, P. J. Hirschfeld, H. R. Ott, J. Beuers, Z. Fisk, and J. L. Smith, "Anomalous temperature dependence of the magnetic field penetration depth in superconducting $\mathrm{UBe}_{13}$," Zeitschrift fr Physik B Condensed Matter, vol. 64, pp. 175-188, 1986.

[135] H. Padamsee, J. E. Neighbor, and C. A. Shiffman, "Quasiparticle phenomenology for thermodynamics of strong-coupling superconductors," Journal of Low Temperature Physics, vol. 12, p. 387, 1973.

[136] C. Ren, Z.-S. Wang, H.-Q. Luo, H. Yang, L. Shan, and H.-H. Wen, "Evidence for two energy gaps in superconducting $\mathrm{Ba}_{0.6} \mathrm{~K}_{0} \cdot 4 \mathrm{Fe}_{2} \mathrm{As}_{2}$ single crystals and the breakdown of the Uemura plot," Physical Review Letters, vol. 101, p. 257006, 2008. 
[137] Y. J. Uemura, V. J. Emery, A. R. Moodenbaugh, M. Suenaga, D. C. Johnston, A. J. Jacobson, J. T. Lewandowski, J. H. Brewer, R. F. Kiefl, S. R. Kreitzman, G. M. Luke, T. Riseman, C. E. Stronach, W. J. Kossler, J. R. Kempton, X. H. Yu, D. Opie, , and H. E. Schone, “Systematics in the thermoelectric power of high- $t_{c}$ oxides," Physical Review B, vol. 38, no. 1, p. 909(R), 1988.

[138] Y. M. Dai, B. Xu, B. Shen, H. H. Wen, X. G. Qiu, and R. P. S. M. Lobo, "Optical conductivity of $\mathrm{Ba}_{0.6} \mathrm{~K}_{0} .4 \mathrm{Fe}_{2} \mathrm{As}_{2}$ : The effect of in-plane and out-of-plane doping in the superconducting gap," Europhysics Letters, vol. 104, p. 47006, 2013.

[139] J. P. Hill, B. J. Sternlieb, D. Gibbs, C. Detlefs, A. I. Goldman, C. Stassis, P. C. Canfield, and B. K. Chol, "Incommensurate antiferromagnetism in the intermetallic superconductor $\mathrm{HoNi}_{2} \mathrm{~b}_{2} \mathrm{c}$," Physical Review $B$, vol. 53, no. 6, p. 3487, 1996.

[140] E. F. Talantsev, W. P. Crump, J. G. Storey, and J. L. Tallon, “London penetration depth and thermal fluctuations in the sulphur hydride 203 K superconductor," Annalen der Physik (Berlin), vol. 529, no. 3, 2016.

[141] T. Timusk and B. Statt, "The pseudogap in high-temperature superconductors: an experimental survey," Reports on Progress in Physics, vol. 62, no. 1, p. 61122, 1999.

[142] J. L. Tallon, J. G. Storey, and J. W. Loram, "Fluctuations and critical temperature reduction in cuprate superconductors," Physical Review B, vol. 83, p. 092502, 2011.

[143] J. W. Loram, J. Luo, J. R. Cooper, W. Y. Liang, and J. L. Tallon, “Evidence on the pseudogap and condensate from the electronic specific heat," Journal of Physics and Chemistry of Solids, vol. 62, pp. 59-64, 2001. 
[144] Y. Mei, S. M. Green, G. G. Reynolds, T. Wiczynski, H. L. Luo, and C. Politis, "Magnetic properties of $\mathrm{GdBa}_{2} \mathrm{Cu}_{3} \mathrm{O}_{7-x}$ in the normal and superconducting states," Zeitschrift für Physik B Condensed Matter, vol. 67, pp. 303-305, 1987.

[145] N. Giordano, "Superconductivity and dissipation in small-diameter Pb-In wires," Physical Review B, vol. 43, p. 160, 1991.

[146] Y. Chen, Y. Lin, S. D. Snyder, and A. M. Goldman, "Stabilization of superconductivity by magnetic field in out-of-equilibrium nanowires," Physical Review B, vol. 83, p. 054505, 2011.

[147] Y. Chen, Y. Lin, S. D. Snyder, A. M. Goldman, and A. Kamenev, “Dissipative superconducting state of non-equilibrium nanowires," $\mathrm{Na}$ ture Physics, vol. 10, p. 567571, 2014.

[148] V. Ambegaokar and A. Baratoff, "Tunneling between superconductors," Physical Review Letters, vol. 11, p. 104, 1963.

[149] V. Ambegaokar and A. Baratoff, "Tunneling between superconductors," Physical Review Letters, vol. 10, p. 408, 1963.

[150] E. L. Wolf, Principles of Electron Tunneling Spectroscopy. New York: Oxford University Press, 1985.

[151] C. P. Poole, H. A. Farach, R. J. Creswick, and R. Prozorov, Superconductivity. London: Academic Press, second edition ed., 2007.

[152] C. A. Bryant and P. H. Keesom, "Low-temperature specific heat of indium and tin," Physical Review, vol. 123, no. 2, p. 491, 1961.

[153] F. B. Hagedorn, "Silsbee-limit critical currents in a 1700å film of tin," Physical Review Letters, vol. 12, no. 12, p. 322, 1964.

[154] K. E. Kihlstrom, R. W. Simon, and S. A. Wolf, “Tunneling $\alpha^{2} f(\omega)$ from sputtered thin-film NbN," Physical Review B, vol. 32, no. 3, p. 1843(R), 1985. 
[155] C. Geibel, H. Rietschel, A. Junod, M. Pelizzone, and J. Muller, “Electronic properties, phonon densities of states and superconductivity in $\mathrm{Nb}_{1-x} \mathrm{~V}_{x} \mathrm{~N}$," Journal of Physics F: Metal Physics, vol. 15, no. 2, p. 405, 1985.

[156] A. Y. Rusanov, M. B. S. Hesselberth, and J. Aarts, “Depairing currents in superconducting films of $\mathrm{Nb}$ and amorphous MoGe," Physical Review B, vol. 70, no. 2, p. 024510, 2004.

[157] H. Tashiro, J. M. Graybeal, D. B. Tanner, E. J. Nicol, J. P. Carbotte, , and G. L. Carr, "Unusual thickness dependence of the superconducting transition of $\alpha$-MoGe thin films," Physical Review B, vol. 78, no. 1, p. 014509, 2008.

[158] J. S. Urbach, W. R. White, M. R. Beasley, and A. Kapitulnik, "Specific heat of a superconducting multilayer: 2D fluctuations and 2D-0D crossover," Physical Review Letters, vol. 69, no. 16, p. 2407, 1992.

[159] Q. Huang, J. F. Zasadzinski, N. Tralshawala, K. E. Gray, D. G. Hinks, J. L. Peng, and R. L. Greene, "Tunnelling evidence for predominantly electronphonon coupling in superconducting $\mathrm{Ba}_{1-x} \mathrm{~K}_{x} \mathrm{BiO}_{3}$ and $\mathrm{Nd}_{2-x} \mathrm{Ce}_{x} \mathrm{CuO}_{4-y}$," Nature, vol. 347, pp. 369-372, 1990.

[160] B. F. Woodfield, D. A. Wright, R. A. Fisher, N. E. Phillips, and H. Y. Tang, "Superconducting-normal phase transition in $\mathrm{Ba}_{1-x} \mathrm{~K}_{x} \mathrm{BiO}_{3}, x$ = 0.40, 0.47," Physical Review Letters, vol. 83, no. 22, p. 4622, 1999.

[161] M. Kosugi, J. Akimitsu, T. Uchida, M. Furuya, Y. Nagata, and T. Ekino, "Tunneling studies on $\mathrm{Ba}_{1-x} \mathrm{~K}_{x} \mathrm{BiO}_{3}$ single crystals with $t_{c}$ = 15-30 K," Physica C: Superconductivity, vol. 229, pp. 389-395, 1994.

[162] G. K. Panova, A. A. Shikov, B. I. Savel'ev, A. P. Zhernov, N. V. Anshukova, A. I. Golovashkin, A. I. Ivanova, and A. P. Rusakov, "Specific heat of the noncuprate oxide superconductor $\mathrm{Ba}_{0.6} \mathrm{~K}_{0.4} \mathrm{BiO}_{3}$ 
in magnetic fields," Journal of Experimental and Theoretical Physics, vol. 76, pp. 302-307, 1993.

[163] T. Uchida, S. Nakamura, N. Suzuki, Y. Nagata, W. D. Mosley, M. D. Lan, P. Klavins, and R. N. Shelton, "Effect of growth conditions on the superconductivity of $\mathrm{Ba}_{0.6} \mathrm{~K}_{0.4} \mathrm{BiO}_{3}$ crystals," Physica C: Superconductivity, vol. 215, pp. 350-358, 1993.

[164] E. J. Ansaldo, Z. R. Wang, J. H. Cho, D. C. Johnston, and R. M. Riseman, "Magnetic penetration depth of $\mathrm{Ba}_{0} .625 \mathrm{~K}_{0} .375 \mathrm{BiO}_{3}$," Physica C: Superconductivity, vol. 185-189, pp. 1889-1890, 1991.

[165] S. C. Wimbush and B. Holzapfel, "Angular anisotropy of the upper critical field in $\mathrm{HoNi}_{2} \mathrm{~B}_{2} \mathrm{C}$," Physica Status Solidi C, vol. 3, no. 9, pp. 3007-3010, 2006.

[166] S. C. Wimbush, L. Schultz, and B. Holzapfel, "Critical current in $\mathrm{YNi}_{2} \mathrm{~B}_{2} \mathrm{C}$ and $\mathrm{HoNi}_{2} \mathrm{~B}_{2} \mathrm{C}$ thin films," Physica C: Superconductivity, vol. 388-389, pp. 191-192, 2003.

[167] Y. G. Naidyuk, O. E. Kvitnitskaya, I. K. Yanson, G. Fuchs, K. Nenkov, A. Wälte, G. Behr, D. Souptel, and S. L. Drechsler, "Point-contact spectroscopy of the antiferromagnetic superconductor $\mathrm{HoNi}_{2} \mathrm{~B}_{2} \mathrm{C}$," Physica C: Superconductivity, vol. 460-462, no. 1, pp. 105-106, 2007.

[168] S. C. Wimbush, L. Schultz, and B. Holzapfel, "Angular anisotropy of the upper critical field in $\mathrm{YNi}_{2} \mathrm{~B}_{2} \mathrm{C}$," Physica C: Superconductivity, vol. 408-410, pp. 83-84, 2004.

[169] C. P. Poole, H. A. Farach, R. J. Creswick, and R. Prozorov, Superconductivity. London: Academic Press, second edition ed., 2007.

[170] R. Prozorov, E. R. Yacoby, I. Felner, and Y. Yeshurun, "Magnetic properties of $\mathrm{YNi}_{2} \mathrm{~B}_{2} \mathrm{C}$ superconductor," Physica C: Superconductivity, vol. 233, pp. 367-372, 1994. 
[171] X. Lu, W. K. Park, S. Yeo, K.-H. Oh, S.-I. Lee, S. L. Budko, P. C. Canfield, and L. H. Greene, "Superconducting order parameter in nonmagnetic borocarbides $\mathrm{RNi}_{2} \mathrm{~B}_{2} \mathrm{C}(\mathrm{R}=\mathrm{Y}, \mathrm{Lu})$ probed by point-contact Andreev reflection spectroscopy," Physical Review B, vol. 83, no. 10, p. 104519, 2011.

[172] R. Puźniak, R. Usami, K. Isawa, and H. Yamauchi, "Superconducting-state thermodynamic parameters and anisotropy of $\mathrm{HgBa}_{2} \mathrm{Ca}_{n-1} \mathrm{Cu}_{n} \mathrm{O}_{y}$ by reversible magnetization measurements," Physical Review B, vol. 52, p. 3756, 1995.

[173] L. KrusinElbaum, C. C. Tsuei, and A. Gupta, "High current densities above $100 \mathrm{~K}$ in the high-temperature superconductor $\mathrm{HgBa}_{2} \mathrm{CaCu}_{2} \mathrm{O}_{6+\delta}$," Nature, vol. 373, p. 679681, 1995.

[174] "Electronic Raman scattering on the underdoped $\mathrm{HgBa}_{2} \mathrm{Ca}_{2} \mathrm{Cu}_{3} \mathrm{O}_{8+\delta}, " 2000$.

[175] J. R. Thompson, J. G. Ossandon, D. K. Christen, M. Paranthaman, E. D. Specht, and Y. C. Kim, "Comparative study of the characteristic length scales and fields of Hg-based high- $t_{c}$ superconductors," Physical Review B, vol. 54, p. 7505, 1996.

[176] H. Ri, R. Gross, F. Gollnik, A. Beck, R. P. Huebener, P. Wagner, and H. Adrian, "Nernst, Seebeck, and Hall effects in the mixed state of $\mathrm{YBa}_{2} \mathrm{Cu}_{3} \mathrm{O}_{7-\delta}$ and $\mathrm{Bi}_{2} \mathrm{Sr}_{2} \mathrm{CaCu}_{2} \mathrm{O}_{8+x}$ thin films: A comparative study," Physical Review B, vol. 50, p. 3312, 1994.

[177] P. Wagner, F. Hillmer, U. Frey, and H. Adrian, “Thermally activated flux movement and critical transport current density in epitaxial $\mathrm{Bi}_{2} \mathrm{Sr}_{2} \mathrm{CaCu}_{2} \mathrm{O}_{8+x}$," Physical Review B, vol. 49, p. 13184, 1994.

[178] A. Suzuki and M. Suzuki, "Tunneling studies of pseudo-gap in $\mathrm{Bi}_{2} \mathrm{Sr}_{2} \mathrm{CaCu}_{1} .94 \mathrm{Zn}_{0} .06 \mathrm{O}_{8-y}$," Physica C: Superconductivity, vol. 378381, pp. 306-310, 2002. 
[179] J. Mosqueira, E. G. Miramontes, C. Torrón, J. A. Campá, I. Rasines, and F. Vidal, "Thermal fluctuation effects on the magnetization above and below the superconducting transition in $\mathrm{Bi}_{2} \mathrm{Sr}_{2} \mathrm{CaCu}_{2} \mathrm{O}_{8}$ crystals in the weak magnetic field limit," Physical Review B, vol. 53, p. 15272, 1996.

[180] N. Hudákova, P. Samuely, P. Szabó, V. Plecháček, K. Knižek, and D. Sedmidubský, "Scaling of the superconducting order parameter in Bi cuprates with $t_{c}$ " Physica C: Superconductivity, vol. 246, pp. 306310, 2002.

[181] Q. Li, M. Suenaga, J. Gohng, D. K. Finnemore, T. Hikata, and K. Sato, "Reversible magnetic properties of c-axis-oriented superconducting $\mathrm{Bi}_{2} \mathrm{Sr}_{2} \mathrm{Ca}_{2} \mathrm{Cu}_{3} \mathrm{O}_{10}$," Physical Review B, vol. 46, p. 3195(R), 1992.

[182] J. Hänisch, A. Attenberger, B. Holzapfel, and L. Schult, "Electrical transport properties of $\mathrm{Bi}_{2} \mathrm{Sr}_{2} \mathrm{Ca}_{2} \mathrm{Cu}_{3} \mathrm{O}_{10+\delta}$ thin film [001] tilt grain boundaries," Physical Review B, vol. 65, p. 052507, 2002.

[183] M. Weigand, M. Eisterer, E. Giannini, and H. Weber, “Mixed state properties of $\mathrm{Bi}_{2} \mathrm{Sr}_{2} \mathrm{Ca}_{2} \mathrm{Cu}_{3} \mathrm{O}_{10+\delta}$ single crystals before and after neutron irradiation," Physical Review B, vol. 81, p. 014516, 2010.

[184] H.-J. Kim, P. Chowdhury, I.-S. Jo, and S.-I. Lee, “Equilibrium magnetization of $\mathrm{Tl}_{2} \mathrm{Ba}_{2} \mathrm{CaCu}_{2} \mathrm{O}_{8+\delta}$ single crystals," Physical Review B, vol. 66, p. 134508, 2002.

[185] Q. Huang, J. F. Zasadzinski, K. E. Gray, E. D. Bukowski, and D. M. Ginsberg, "Point-contact tunneling study of the normal and superconducting states of $\mathrm{Tl}_{2} \mathrm{Ba}_{2} \mathrm{CaCu}_{2} \mathrm{O}_{x}$," Physica C: Superconductivity, vol. 161, pp. 141-144, 1989.

[186] A. Junod, D. Eckert, G. Triscone, J. Muller, and V. Lee, "Specific heat $(1330 \mathrm{~K})$, Meissner effect and magnetic susceptibility of 
$\mathrm{Tl}_{2} \mathrm{Ba}_{2} \mathrm{Ca}_{2} \mathrm{Cu}_{3} \mathrm{O}_{10}, \mathrm{Tl}_{2} \mathrm{Ba}_{2} \mathrm{CaCu}_{2} \mathrm{O}_{8}$ and $\mathrm{Tl}_{2} \mathrm{Ba}_{2} \mathrm{CuO}_{6}$ ceramic samples," Physica C: Superconductivity, vol. 159, pp. 215-225, 1989.

[187] J. T. Moonen and H. B. Brom, "Vortex-mobility determined NMR properties in highly anisotropic high- $t_{c}$ superconductors II. experiment on $\mathrm{Tl}_{2} \mathrm{Ba}_{2} \mathrm{CaCu}_{2} \mathrm{O}_{8}$," Physica C: Superconductivity, vol. 244, pp. 10-20, 1995.

[188] Y. Dagan, R. Krupke, and G. Deutscher, "Determination of the superconducting gap in $\mathrm{YBa}_{2} \mathrm{Cu}_{3} \mathrm{O}_{7-\delta}$ by tunneling experiments under magnetic fields," Physical Review B, vol. 62, p. 146, 2000.

[189] J. Loram, K. Mirza, and P. Freeman, "The electronic specific heat of $\mathrm{YBa}_{2}\left(\mathrm{Cu}_{1-x} \mathrm{Zn}_{x}\right)_{3} \mathrm{O}_{7}$ from $1.6 \mathrm{k}$ to $300 \mathrm{k,"}$, Physica C, vol. 171, pp. 243256, 1990.

[190] J. L. Tallon, C. Bernhard, U. Binninger, A. Hofer, G. V. M. Williams, E. J. Ansaldo, J. I. Budnick, and C. Niedermayer, "In-plane anisotropy of the penetration depth due to superconductivity on the cu-o chains in $\mathrm{YBa}_{2} \mathrm{Cu}_{3} \mathrm{O}_{7-\delta}, \mathrm{Y}_{2} \mathrm{Ba}_{4} \mathrm{Cu}_{7} \mathrm{O}_{15-\delta}$, and $\mathrm{YBa}_{2} \mathrm{Cu}_{4} \mathrm{O}_{8}$," Physical Review Letters, vol. 74, p. 1008, 1995.

[191] C. P. Poole, H. A. Farach, R. J. Creswick, and R. Prozorov, Superconductivity. London: Academic Press, second edition ed., 2007.

[192] F. Rothwarf and L. Dubeck, "The pressure dependence of superconducting energy gaps from high pressure critical field measurements," Solid State Communications, vol. 13, pp. 1645-1649, 1973.

[193] I. Bonalde, B. D. Yanoff, M. Salamon, and E. Chia, "Nonlocality in superconducting metals: an ultra-high precision magnetic penetration depth study," Physica B: Condensed Matter, vol. 329-333, pp. 1373-1374, 2003. 
[194] Y. Sun, J. Wang, W. Zhao, M. Tian, M. Singh, and M. H. W. Chan, "Voltage-current properties of superconducting amorphous tungsten nanostrips," Scientific Reports, vol. 3, p. 2307, 2013.

[195] C. T. Rao, L. W. Dubeck, and F. Rothwarf, "Superconducting energy gaps from magnetization measurements: Pb-In system," Physical Review B, vol. 7, p. 1866, 1973.

[196] N. R. Groll, J. A. Klug, C. Cao, S. Altin, H. Claus, N. G. Becker, J. F. Zasadzinski, M. J. Pellin, and T. Proslier, "Tunneling spectroscopy of superconducting MoN and NbTiN grown by atomic layer deposition," Applied Physics Letters, vol. 104, p. 092602, 2014.

[197] P. Larsson, B. Nilsson, and Z. G. Ivanov, "Fabrication and transport measurements of $\mathrm{YBa}_{2} \mathrm{Cu}_{3} \mathrm{O}_{7-x}$ nanostructures," Journal of Vacuum Science and Technology B: Microelectronics and Nanometer Structures Processing, Measurement, and Phenomena, vol. 18, p. 25, 2000.

[198] S. Nawaz, R. Arpaia, T. Bauch, and F. Lombardi, "Approaching the theoretical depairing current in $\mathrm{YBa}_{2} \mathrm{Cu}_{3} \mathrm{O}_{7-x}$ nanowires," Physica C: Superconductivity, vol. 495, pp. 33-38, 2013.

[199] E. F. Talantsev, N. M. Strickland, S. C. Wimbush, and W. P. Crump, "The onset of dissipation in high-temperature superconductors: Self-field experiments," AIP Advances, vol. 7, p. 125230, 2017.

[200] E. F. Talantsev, A. E. Pantoja, W. P. Crump, and J. L. Tallon, “Current distribution across type II superconducting films: a new vortex-free critical state," Scientific Reports, vol. 8, p. 1716, 2018.

[201] D.-H. Wu and S. Sridhar, "Pinning forces and lower critical fields in $\mathrm{YBa}_{2} \mathrm{Cu}_{3} \mathrm{O}_{y}$ crystals: Temperature dependence and anisotropy," Physical Review Letters, vol. 65, p. 2074, 1990. 
[202] V. A. Finkel and V. V. Derevyanko, "Lower critical fields of textured high-temperature superconductors. III. Experimental study of the anisotropy of the fields $h_{c 1}$ of the HTSC $\mathrm{YBa}_{2} \mathrm{Cu}_{3} \mathrm{O}_{7-\delta}$," Low Temperature Physics, vol. 26, p. 92, 2000.

[203] Y. Sun and K. Maki, "Impurity effects in $d$-wave superconductors," Physical Review B, vol. 51, no. 9, p. 6059, 1995.

[204] H. Suhl and B. T. Matthias, "Impurity scattering in superconductors," Physical Review, vol. 114, no. 4, p. 977, 1959.

[205] C. Panagopoulos, J. R. Cooper, N. Athanassopoulou, and J. Chrosch, "Effects of $\mathrm{Zn}$ doping on the anisotropic penetration depth of $\mathrm{YBa}_{2} \mathrm{Cu}_{3} \mathrm{O}_{7}$," Physical Review B, vol. 54, no. 18, p. R12721(R), 1996.

[206] M. E. Simon and C. M. Varma, "Magnetic impurities in $d$-wave superconductors," Physical Review B, vol. 60, no. 13, p. 9744, 1999.

[207] C. Bernhard, J. L. Tallon, C. Bucci, R. D. Renzi, G. Guidi, G. V. M. Williams, and C. Niedermayer, "Suppression of the superconducting condensate in the high- $T_{c}$ cuprates by $\mathrm{Zn}$ substitution and overdoping: Evidence for an unconventional pairing state," Physical Review Letters, vol. 77, no. 11, p. 2304, 1996.

[208] C. P. Poole, H. A. Farach, R. J. Creswick, and R. Prozorov, Superconductivity. London: Academic Press, second edition ed., 2007.

[209] P. J. Hirschfeld and N. Goldenfeld, "Effect of strong scattering on the low-temperature penetration depth of a $d$-wave superconductor," Physical Review B, vol. 48, no. 6, p. 4219(R), 1993.

[210] P. Arberg and J. P. Carbotte, "Effect of the impurity scattering on the zero-temperature penetration depth in $d_{x^{2}-y^{2}}$ symmetry," Physical Review B, vol. 50, no. 5, p. 3250, 1994. 
[211] I. Kosztin and A. J. Leggett, "Nonlocal effects on the magnetic penetration depth in $d$-wave superconductors," Physical Review Letters, vol. 79, no. 1, p. 135, 1997.

[212] A. Carrington, F. Manzano, R. Prozorov, R. W. Giannetta, N. Kameda, and T. Tamegai, "Evidence for surface andreev bound states in cuprate superconductors from penetration depth measurements," Physical Review Letters, vol. 86, no. 6, p. 1074, 2001.

[213] J. L. Tallon, C. Bernhard, G. V. M. Williams, , and J. W. Loram, “Zninduced $T_{c}$ reduction in high- $T_{c}$ superconductors: Scattering in the presence of a pseudogap," Physical Review Letters, vol. 79, no. 26, p. 5294, 1997.

[214] E. F. Talantsev, W. P. Crump, J. Island, Y. Xing, Y. Sun, J. Wang, and J. L. Tallon, "On the origin of critical temperature enhancement in atomically thin superconductors," 2D Materials, vol. 4, no. 2, 2017.

[215] A. Piriou, Y. Fasano, E. Giannini, and O. Fischer, "Effect of oxygendoping on $\mathrm{Bi}_{2} \mathrm{Sr}_{2} \mathrm{Ca}_{2} \mathrm{Cu}_{3} \mathrm{O}_{10+\delta}$ vortex matter: Crossover from electromagnetic to Josephson interlayer coupling," Physical Review B, vol. 77, p. 184508, 2000.

[216] W. Shi, J. Ye, Y. Zhang, R. Suzuki, M. Yoshida, J. Miyazaki, N. Inoue, Y. Saito, and Y. Iwasa, "Superconductivity series in transition metal dichalcogenides by ionic gating," Scientific Reports, vol. 5, p. 12534, 2015.

[217] X. Xi, H. Berger, L. Forr, J. Shan, and K. F. Mak, "Gate tuning of electronic phase transitions in two-dimensional $\mathrm{NbSe}_{2}$," Physical Review Letters, vol. 117, p. 106801, 2016.

[218] D. Costanzo, S. Jo, H. Berger, and A. F. Morpurgo, "Gate-induced superconductivity in atomically thin $\mathrm{MoS}_{2}$ crystals," Nature Nanotechnology, vol. 11, pp. 339-344, 2016. 
[219] A. Fłte, L. Rossi, A. Augieri, and C. Senatore, "Ionic liquid gating of ultra-thin $\mathrm{YBa}_{2} \mathrm{Cu}_{3} \mathrm{O}_{7 x}$ films," Applied Physics Letters, vol. 109, p. 192601, 2016.

[220] G. Dubuis, Y. Yacoby, H. Zhou, X. He, A. T. Bollinger, D. Pavuna, R. Pindak, and I. Boovi, "Oxygen displacement in cuprates under ionic liquid field-effect gating," Scientific Reports, vol. 6, p. 32378, 2016. 